Clustering mechanism of oxocarboxylic acids involving hydration reaction : Implications for the atmospheric models

\author{
Liu, Ling
}

2018-06-07

Liu , L , Kupiainen-Maatta , O , Zhang , H , Li , H , Zhong , J , Kurten , T , Vehkamaki , H , Zhang , S , Zhang , Y , Ge , M , Zhang , X \& Li , Z 2018, ' Clustering mechanism of oxocarboxylic acids involving hydration reaction : Implications for the atmospheric models ', Journal of Chemical Physics , vol. 148 , no. 21 , 214303 . https://doi.org/10.1063/1.5030665

http://hdl.handle.net/10138/274994

https://doi.org/10.1063/1.5030665

acceptedVersion

Downloaded from Helda, University of Helsinki institutional repository.

This is an electronic reprint of the original article.

This reprint may differ from the original in pagination and typographic detail.

Please cite the original version. 


\section{Clustering Mechanism of Oxocarboxylic Acids Involving Hydration Reaction: Implications for the Atmospheric Models}

Ling Liu, ${ }^{1}$ Oona Kupiainen-Määttä, ${ }^{2}$ Haijie Zhang, ${ }^{1}$ Hao Li, ${ }^{1}$ Jie Zhong, ${ }^{3}$ Theo Kurtén, ${ }^{4}$ Hanna Vehkamäki, ${ }^{2}$ Shaowen Zhang, ${ }^{1}$ Yunhong Zhang, ${ }^{1}$ Maofa Ge, ${ }^{5}$ Xiuhui Zhang, ${ }^{1}$ a) and Zesheng $\mathrm{Li}^{1, \mathrm{~b}}$ )

1) Key Laboratory of Cluster Science, Ministry of Education of China, School of Chemistry and Chemical Engineering, Beijing Institute of Technology, Beijing 100081, China

${ }^{2)}$ Institute for Atmospheric and Earth System Research/Physics, University of Helsinki, PO Box 64 (Gustaf Hällströmin katu 2a), FI-00014, Helsinki, Finland

${ }^{3)}$ Department of Chemistry, University of Nebraska-Lincoln, Lincoln, NE, USA 68588

${ }^{4)}$ Institute for Atmospheric and Earth System Research/Chemistry, University of Helsinki, PO Box 64 (Gustaf Hällströmin katu 2a), FI-00014, Helsinki, Finland

5) Beijing National Laboratory for Molecular Sciences (BNLMS), State Key Laboratory for Structural Chemistry of Unstable and Stable Species, Institute of Chemistry, Chinese Academy of Sciences, Beijing 100190, China

(Dated: 4 May 2018) 
The formation of atmospheric aerosol particles from condensable gases is a dominant source of particulate matter in the boundary layer, but the mechanism is still ambiguous. During the clustering process, precursors with different reactivities can induce various chemical reactions in addition to the formation of hydrogen bonds. However, the clustering mechanism involving chemical reactions is rarely considered in most of the nucleation process models. Oxocarboxylic acids are common compositions of secondary organic aerosol, but the role of oxocarboxylic acids in secondary organic aerosol formation is still not fully understood. In this paper, glyoxylic acid, the simplest and the most abundant atmospheric oxocarboxylic acids, has been selected as a representative example of oxocarboxylic acids in order to study the clustering mechanism involving hydration reaction using Density Functional Theory combined with the Atmospheric Clusters Dynamic Code. The hydration reaction of glyoxylic acid can occur either in the gas phase or during the clustering process. In atmospheric conditions, the total conversion ratio of glyoxylic acid to its hydration reaction product (2,2-dihydroxyacetic acid) in both gas phase and clusters can be up to $85 \%$, and the product can further participate in the clustering process. The differences in cluster structures and properties induced by the hydration reaction lead to significant differences in cluster formation rates and pathways at relatively low temperatures.

a) Electronic mail: zhangxiuhui@bit.edu.cn

b)Electronic mail: zeshengli@bit.edu.cn 


\section{I. INTRODUCTION}

2

Atmospheric aerosols have significant impacts on climate, weather and human health. ${ }^{1,2}$ However, the formation mechanisms and composition of atmospheric aerosols are still not fully understood, and this constitutes one of the largest uncertainties in current atmospheric models. ${ }^{3,4}$ There is compelling evidence that sulfuric acid (SA), water $(\mathbf{W})$, ammonia (A) or amines can play key roles in atmospheric new particle formation (NPF), but these compounds are still not efficient enough to explain NPF in all the environments where it has been observed. Recently, numerous atmospheric observations and theoretical studies have shown that organic acids can also enhance NPF, ${ }^{5-15}$ However, there are potentially tens of thousands of different atmospheric organic species with varying properties, which makes the exact chemical composition of clusters containing organic molecules highly speculative. Furthermore, different organics have different chemical reactivities. Thus, NPF may be driven not only by clustering processes, but also by various other complex and condition-dependent atmospheric chemical reactions. ${ }^{16-23}$, which can influence the physical and chemical processes of NPF ${ }^{24-26}$. This makes the assessment of the role of organic compounds in the NPF process very complicated.

Oxocarboxylic acids are one of the most common organic species group found in secondary organic aerosol (SOA) in diverse environments. Experimental and theoretical studies have shown that the equilibrium reaction between carbonyl groups and the corresponding geminal diols can occur in the gas phase, ${ }^{27-30}$ indicating that the gas-phase hydration reaction of oxocarboxylic acids may potentially occur along with the clustering process driving NPF. As the water concentration in the atmosphere is typically 8-10 orders of magnitude higher than that of other condensing species, ${ }^{31}$ such hydration reactions are potentially of great 
significance. However, in most present atmospheric aerosol formation models, the hydration reactions of oxocarboxylic acids have been neglected due to the lack of information on them. This may contribute to the discrepancy between measured and modeled results. ${ }^{3,4}$ In this study, we seek to understand the kinetics of atmospheric clustering processes involving hydration reactions of oxocarboxylic acids under different atmospheric environments (different precursor concentrations, relative humidities (RHs) and temperatures).

Experiments show that the gas-phase hydration reaction of glyoxylic acid (GA), the simplest and the most abundant oxocarboxylic acids in the atmosphere, ${ }^{32,33}$ is able to form its geminal diol $(\mathbf{G W}) .{ }^{27}$ Our previous theoretical study has also shown that this process can be effectively catalyzed by different catalysts (SA, W or A), among which SA is the most effective, lowering the activation free energy barrier from 38.56 to 9.48 $\mathrm{kcal} / \mathrm{mol} .{ }^{34}$ Therefore, GA has been selected as a representative example of oxocarboxylic acids in order to study the clustering mechanism involving hydration reaction. A combination of Density Functional Theory and the Atmospheric Clusters Dynamic Code (ACDC) $)^{35}$ has been used. As the hydrated clusters play an important role in cluster formation and growth ${ }^{36}$ water $(\mathbf{W})$-containing clusters are included in our study. The studied system is $\left.(\mathbf{G A} / \mathbf{G W})_{x} \cdot \mathbf{S A}\right)_{y} \cdot \mathbf{A}_{z} \cdot \mathbf{W}_{0-}$, where $x$ is the number of $\mathbf{G A} / \mathbf{G W}$ molecules in the cluster, $y$ is the number of $\mathbf{S A}$ molecules in the cluster, $z$ is the number of $\mathbf{A}$ molecules in the clusters, $x+y \geq z$ (i.e. only clusters that are acidic or chemically neutral are studied), and $1 \leq x+y+z \leq 3$ (i.e. the studied clusters contain at most three molecules other than water). The maximum number of water molecules in the cluster, $n$, depends on the cluster type, and has been chosen so that all hydrates with relative abundance higher than $5 \%$ have been included. There is always maximally one $\mathbf{G A}$ or $\mathbf{G W}$ molecule in the cluster. 


\section{COMPUTAIONAL METHODS}

\section{A. Quantum chemical calculations}

The initial guesses for all the structures of clusters were generated by the ABCluster 37,38 program, which searches for global and local minima of molecular clusters using the artificial bee colony $(\mathrm{ABC})$ algorithm. In ABCluster, water molecules were described by the TIP4P model and other molecules were described by the CHARMM36 force field. ${ }^{39}$ First, about 1000 structures (for each cluster stoichiometry) were generated with ABCluster, and then these structures were optimized using the PM7 semiempirical method ${ }^{40,41}$ using MOPAC2016 ${ }^{42}$. Second, up to 100 structures with relatively low energies were selected for subsequently optimization with the M06-2X $\mathrm{X}^{43,44}$ density functional and a $6-31+\mathrm{G}^{*}$ basis set. Third, the 10 best of the resulting structures were further re-optimized by the M06-2X density functional with a $6-311++\mathrm{G}(3 \mathrm{df}, 3 \mathrm{pd})$ basis set ${ }^{45}$. The M06-2X functional is one of the most successful functionals in describing noncovalent interactions ${ }^{43}$ and it has been successfully used to model the thermochemistry and equilibrium structures of atmospheric clusters. ${ }^{46,47}$ The 6-311++G(3df,3pd) basis set was chosen based on its common use for atmospherically relevant clusters $^{48-51}$ and its excellent performance to estimate cluster properties when used in conjunction with the M06-2X functional. ${ }^{46}$ We checked that the stable structures had positive vibrational frequencies. All the quantum chemistry calculations were performed using the Gaussian 09 program package. ${ }^{52}$

In additon, topological analysis was performed using atoms in molecules (AIM) theory with the Multiwfn package ${ }^{53}$ to study the nature of hydrogen bonds. The wavefunctions (technically, electron densities) computed at the M06-2X/6-311++G(3df,3pd) level of theory were used to calculate the electron density $\rho$ and Laplacian $\nabla^{2} \rho$ at the bond critical points 
(BCPs).

\section{B. Atmospheric Cluster Dynamics Code (ACDC) kinetic model}

The hydration reaction and clustering process of glyoxylic acids may coexist and compete against each other in the real atmosphere environment. Thus, it is necessary for cluster kinetic models to take into account the relevant chemical reactions to fully simulate the real NPF process in the atmosphere. The structural, thermodynamic and kinetic data generated by quantum chemistry calculations were used as input in cluster formation simulations performed using the Atmospheric Cluster Dynamics Code (ACDC) $)^{35,54}$.

The time development of the concentrations of each cluster was solved by integrating numerically the birth-death equations ${ }^{35}$ using the ode15s solver in MATLAB-R2013a program $^{55}$. The birth-death equations can be written as

$$
\begin{aligned}
\frac{d c_{i}}{d t}= & \frac{1}{2} \sum_{j<i} \beta_{j,(i-j)} c_{j} c_{(i-j)}+\sum_{j} \gamma_{(i+j) \rightarrow i} c_{i+j}-\sum_{j} \beta_{i, j} c_{i} c_{j} \\
& -\frac{1}{2} \sum_{j<i} \gamma_{i \rightarrow j} c_{i}+\sum_{j} k_{j \rightarrow i} c_{j}-\sum_{j} k_{i \rightarrow j} c_{i}+Q_{i}-S_{i},
\end{aligned}
$$

where $c_{i}$ is the concentration of cluster $i, \beta_{i, j}$ is the collision coefficient between clusters $i$ and $j, \gamma_{i \rightarrow j}$ is the evaporation coefficient of a molecule or a smaller cluster $j$ from cluster $i, Q_{i}$ is an outside source term of cluster $i$, and $S_{i}$ is other possible sink term of cluster $i$. The coagulation sink coefficient corresponding to coagulation onto pre-existing larger particles was varied in the range of $10^{-3} \mathrm{~s}^{-1}$ to $5 \times 10^{-3} \mathrm{~s}^{-1}$, and the results indicate that our conclusions were not influenced by these variations. ${ }^{56,57}$ Thus, a coagulation sink coefficient of $2.6 \times 10^{-3} \mathrm{~s}^{-1}$ was used for all clusters. ${ }^{56} k_{i \rightarrow j}$ is the reaction rate coefficient of chemical reaction from reactant cluster $i$ to product cluster $j$. The hydration reaction in GA-based 
clusters can be uncatalyzed, or catalyzed by $\mathbf{S A}, \mathbf{W}$ or $\mathbf{A}$, as described in our previous study. ${ }^{34}$ The nature of hydration reaction occurring in a certain $\mathbf{G A}$-based cluster depends on the molecules the cluster contains, and the reaction rate corresponding to the catalyst molecule with the lowest activation free energy barrier has been chosen as the representative rate. (The additional molecules present in the cluster might conceivably also affect the reaction rate, which was neglected in the present study.)

The collision rate coefficient $\beta_{i, j}$ between clusters $i$ and $j$ were calculated using hardsphere collision theory ${ }^{58}$ :

$$
\beta_{i, j}=\pi\left(r_{i}+r_{j}\right)^{2} \sqrt{\frac{8 k_{B} T}{\pi \mu}},
$$

where $r_{i}$ is the radius of cluster $i$ given by the Multiwfn $3.3 .8 \operatorname{program}^{53}, k_{B}$ is the Boltzmann constant, $T$ is the temperature and $\mu=m_{i} m_{j} /\left(m_{i}+m_{j}\right)$ is the reduced mass. The cluster radius is half of the sum of the distance between the center of most distant atoms in cluster given by the Multiwfn 3.3.8 program $^{53}$ and the Van der Walls radii of these atoms.

Evaporation coefficients, $\gamma_{(i+j) \rightarrow i}$, were obtained from the corresponding collision coefficients and the Gibbs free energies of cluster formation using

$$
\gamma_{(i+j) \rightarrow i}=\beta_{i, j} \frac{p_{r e f}}{k_{B} T} \exp \left(\frac{\Delta G_{i+j}-\Delta G_{i}-\Delta G_{j}}{k_{B} T}\right)
$$

where $p_{\text {ref }}$ is the reference pressure (in this case $1 \mathrm{~atm}$ ) at which the formation free energies were calculated, and $\Delta \mathrm{G}_{i+j}$ is the Gibbs free energy of formation of cluster $i+j$ from monomers $i$ and $j$.

The forward and reverse reaction rate coefficients of the chemical reaction were calculated according to the corresponding forward and reverse Gibbs free energy barrier using Eyrings 
equation as ${ }^{59}$

$$
k=\frac{k_{\mathrm{B}} T}{h} e^{-\frac{\Delta G^{\ddagger}}{R T}},
$$

where $k_{B}$ is Boltzmann's constant, $h$ is Planck's constant, and $\Delta \mathrm{G}^{\ddagger}$ is the Gibbs free energy of activation. The Gibbs free energy barrier and rate constants of the forward and reverse reactions are shown in Tables S1-S2 of the supplementary material. ${ }^{34}$

In addition, the tunneling effects could enhance the rate of chemical reaction involving hydrogen atom transfer especially at low temperatures. ${ }^{60}$ Thus, the effect of tunneling on the hydration reaction is considered to correct the corresponding reaction rate constant through the Wigner tunneling correction by a factor $\Gamma(T)$ as

$$
\Gamma(T)=1+\frac{1}{24}\left(\frac{h \nu^{\mp}}{k_{B} T}\right)^{2},
$$

where $h$ is the Planck's constant, $k_{B}$ is Boltzmann's constant, $T$ is the temperature and $\nu^{\mp}$ is the imaginary frequency of the transition state.

Then, the Wigner tunneling factor corrected forward and reverse reaction rate coefficients $\left(k_{i \rightarrow j}, k_{j \rightarrow i}\right)$ of the chemical reaction can be calculated by the tunneling facter as

$$
k_{\mathrm{cor}}=\Gamma k .
$$

The data of $\Gamma(T)$ of all hydration reactions in the present study at different temperatures $(220,240,260,280$ and $300 \mathrm{~K})$ are listed in Table S3 of the supplementary material. It indicates that the maximum value of the $\Gamma(T)$ among all the reactions of the present study is 4.34 (the uncatalyzed hydration reaction) at $220 \mathrm{~K}$, which indicates that the tunneling effect has relatively small influence on the present study. The final hydraton reaction rate 
coefficients are still corrected by the Wigner tunneling factor (Table S4 of the supplementary material) to make the results more accurate.

Atmospheric clusters of hygroscopic species are almost invariably hydrated because the concentration of water in the atmosphere is much larger than that of other condensable species. All the hydrated clusters in the studied system with a relative population of higher than $5 \%$ are considered. Further, the effective collision and evaporation coefficients of clusters need to be computed as a weighted average over the hydrate distributions to get the effective collision and evaporation coefficients.

The hydrate distributions for a cluster $\mathbf{C}$ were calculated as ${ }^{61}$

$$
f\left(\mathbf{C W}_{i}\right)=\frac{\left[\mathbf{C} \mathbf{W}_{i}\right]}{\sum_{j=0}^{j_{\max }}\left[\mathbf{C} \mathbf{W}_{j}\right]},
$$

where $\mathbf{C}$ is a dry molecule or cluster other than water, $\mathbf{W}$ is water, $\mathbf{C W}_{i}$ is the cluster consisting of $\mathbf{C}$ and $i$ water molecules, and $\left[\mathbf{C W}_{i}\right]$ is the concentration of cluster $\mathbf{C} \mathbf{W}_{i}$.

$$
\frac{\left[\mathbf{C W}_{i}\right]}{[\mathbf{C}]}=\left([\mathbf{W}] \frac{k_{B} T}{p_{\text {ref }}}\right)^{i} \exp \left(\frac{\Delta G(\mathbf{C})-\Delta G\left(\mathbf{C W}_{i}\right)}{k_{B} T}\right)
$$

where $[\mathbf{W}]$ is the concentration of water vapor and $p_{\text {ref }}$ is the reference pressure (in this case 1 atm) at which the Gibbs free energies are calculated.

The effective collision coefficients were calculated as

$$
\beta_{e f f}(\mathbf{C}+\mathbf{D})=\sum_{i=0}^{i_{\max }} \sum_{j=0}^{j_{\max }} \beta\left(\mathbf{C W}_{i}+\mathbf{D W}_{j}\right) f\left(\mathbf{C W}_{i}\right) f\left(\mathbf{D W}_{j}\right)
$$


the effective evaporation coefficients similarly as

$$
\gamma_{e f f}(\mathbf{C D} \rightarrow \mathbf{C}+\mathbf{D})=\sum_{i=0}^{i_{\max }} \sum_{j=0}^{j_{\max }} \gamma\left(\mathbf{C D W}_{i+j} \rightarrow \mathbf{C W}_{i}+\mathbf{D W}_{j}\right) f\left(\mathbf{C D W}_{i+j}\right)
$$

and the reaction rate coefficient as

$$
k_{e f f}(\mathbf{C} \rightarrow \mathbf{D})=\sum_{i=1}^{i_{\max }} k_{\mathrm{cor}}\left(\mathbf{C W}_{i} \rightarrow \mathbf{D} \mathbf{W}_{i-1}\right) f\left(\mathbf{C W}_{i}\right)
$$

Thus, when considering the presence of water, the birth-death equations can be written as

$$
\begin{aligned}
\frac{d c_{i}}{d t}= & \frac{1}{2} \sum_{j<i} \beta_{e f f_{j,(i-j)}} c_{j} c_{(i-j)}+\sum_{j} \gamma_{e f f(i+j) \rightarrow i} c_{i+j}-\sum_{j} \beta_{e f f_{i, j}} c_{i} c_{j} \\
& -\frac{1}{2} \sum_{j<i} \gamma_{e f f_{i \rightarrow j}} c_{i}+\sum_{j} k_{e f f_{j \rightarrow i}} c_{j}-\sum_{j} k_{e f f_{i \rightarrow j}} c_{i}+Q_{i}-S_{i} .
\end{aligned}
$$

The concentration of sulfuric acid $\mathbf{S A}, \mathbf{A}$ and $\mathbf{G A}$ are set in the range of $1.0 \times 10^{4} \sim 1.0$ $\times 10^{8}$ molecules $\mathrm{cm}^{-3},{ }^{62-65} 1.0 \times 10^{7} \sim 1.0 \times 10^{11}$ molecules $\mathrm{cm}^{-3},{ }^{62}$ and $1.0 \times 10^{7} \sim 1.0$ $\times 10^{11}$ molecules $\mathrm{cm}^{-3}, 32,66-68$ respectively, which are relevant to the corresponding common atmospheric concentration. The water vapour concentration was adjusted depending on the temperature according to the study on the saturation vapor pressure from Arnold Wexler. ${ }^{69}$ The model runs were performed in the temperature range from $220 \mathrm{~K}$ to $300 \mathrm{~K}$ representing the range from the ground level to the upper free troposphere and $\mathrm{RH}$ ranged from 0 to $100 \%$.

The boundary conditions require the outgrowing clusters to have a favorable composition so that the clusters leaving the studied size range are stable enough not to evaporate back immediately. $(\mathbf{S A})_{3} \cdot \mathbf{A}_{1}$ cluster, with a maximum evaporation rate coefficient of $55 \mathrm{~s}^{-1}$ at 


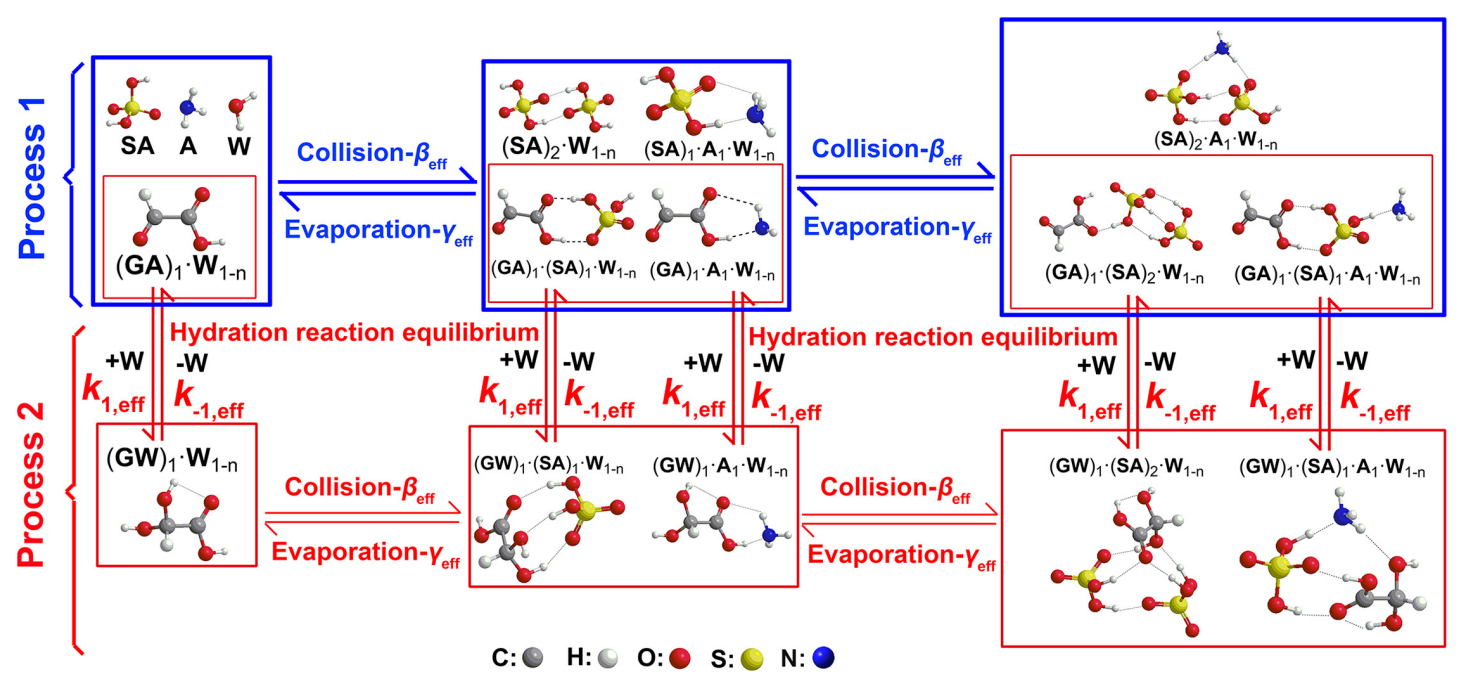

FIG. 1. Modeled clustering processes involving the hydration reaction of $\mathbf{G A}$ and $\mathbf{G A} / \mathbf{G W}$-based clusters. Process 1 (formation of $\mathbf{G A}$-based clusters) and process 2 (formation of $\mathbf{G} \mathbf{W}$-based clusters) are shown in the blue and red line frame, respectively. For simplicity, water molecules in the cluster structures are not shown.

$300 \mathrm{~K}$, is relatively stable enough to resist evaporation (the evaporation rate coefficients of clusters are shown in Table S5 of the supplementary material). Thus, the boundary condition was set to be the $(\mathbf{S A})_{3} \cdot \mathbf{A}_{1}$ cluster (see section 2 in the supplementary material for details). It should be noted that using this relatively small cluster as a boundary condition might overestimate absolute NPF rates, but it is probably sufficient for probing the relative effect of $\mathbf{G A} / \mathbf{G W}$ on cluster formation rate, which is the purpose of this study.

\section{RESULTS AND DISCUSSION}

The clustering process of $\mathbf{G A}$, involving the hydration reaction, includes two kinds of processes (Figure 1). Process 1 is the formation of GA-SA-A containing clusters involving only collision and evaporation steps (without chemical reactions). Process 2 involves not only the collision and evaporation of GW-SA-A containing clusters, but also the hydration reactions of GA-based clusters to form $\mathbf{G W}$-based clusters. 


\section{A. Structure and thermodynamic analysis}

The clustering process of GA considering hydration reaction involves 35 different unhydrated and hydrated clusters (Figures S1-S6 of the supplementary material). The cartesian coordinates of all clusters are listed in Tables S6-S83 of the supplementary material. The atoms in molecules (AIM) analyses were performed to search the bond critical points (BCPs), ring critical points (RCPs) and to calculate electron density $\rho$ and Laplacian $\nabla^{2} \rho$ at the BCPs (Table S84 of the supplementary material). The AIM plots of the clusters without water molecules are shown in Figure S7 of the supplementary material. All these AIM results affirm the existence of intermolecular interaction in clusters. Moreover, the values of $\rho$ and $\nabla^{2} \rho$ at these BCPs range from 0.0115 to 0.0927 a.u. and 0.0288 to 0.1815 a.u. respectively. Most of these values are larger than the critical threshold limits for the formation of hydrogen bond suggested in the littrature (0.002-0.040 a.u. and 0.014-0.139 a.u. for $\rho(\mathrm{BCP})$ and $\nabla^{2} \rho(\mathrm{BCP})$, respectively).$^{70,71}$ These values of $\rho$ and $\nabla^{2} \rho$ thus indicate quite strong hydrogen bond interactions. In addition, there is a large cluster rearrangement after the chemical reaction. The GA molecule binds preferentially to the periphery of the cluster (linear), almost solely by the interaction between its carboxyl group and sulfuric acid. After the reaction, both of the carboxyl group and the hydroxyl group of $\mathbf{G W}$ can interact with all the other clustering constituents inside the cluster (cage-like).

From the Gibbs free energies of formation of clusters (Figure S8 of the supplementary material), it can be seen that the clusters based on $(\mathbf{G A})_{1} \cdot(\mathbf{S A})_{1}$ and $(\mathbf{G A})_{1} \cdot(\mathbf{S A})_{2}$ are more stable than their corresponding $\mathbf{G W}$-based analogues. In contrast, the hydrated clusters based on $(\mathbf{G W})_{1} \cdot(\mathbf{S A})_{1} \cdot \mathbf{A}_{1}$ are much more stable than their corresponding $\mathbf{G A}$-based analogues at different temperatures. 


\section{B. Relative hydration population}

GA and GW are both water soluble organics, and they can influence the hydrate distribution of clusters. The relative hydration population of clusters with varying numbers of water molecules at different relative humidities $(20 \%, 40 \%, 60 \%$ and $100 \%)$ and moderate temperature of $260 \mathrm{~K}$ are shown in Figure 2. The influence of $\mathbf{G A}$ and $\mathbf{G W}$ on the relative hydration population of clusters is different. The addition of $\mathbf{G A}$ on $(\mathbf{S A})_{1} \cdot \mathbf{A}_{1}$ cluster reduces the relative population of clusters with four water molecules and enhancing that of clusters without water molecules, and the addition of $\mathbf{G A}$ on $(\mathbf{S A})_{2}$ cluster reduces the relative population of clusters with three water molecules making the population more evenly. Thus, the addition of $\mathbf{G A}$ reduces the ability of $(\mathbf{S A})_{1} \cdot \mathbf{A}_{1}$ and $(\mathbf{S A})_{2}$ clusters to bind more water molecules. However, addition of $\mathbf{G W}$ on $(\mathbf{S A})_{1} \cdot \mathbf{A}_{1}$ cluster enhances the relative population of clusters with four water molecules reducing that of clusters without water molecules, and the addition of $\mathbf{G W}$ on $(\mathbf{S A})_{2}$ cluster enhances the relative population of clusters with five water molecules. Thus, the addition of $\mathbf{G W}$ enhances the ability of $(\mathbf{S A})_{1} \cdot \mathbf{A}_{1}$ and $(\mathbf{S A})_{2}$ clusters to bind more water molecules. This difference between the influence of $\mathbf{G A}$ and $\mathbf{G W}$ on the cluster hydration population can be explained from the structure characteristic and thermodynamic stability of the corresponding clusters. Two kinds of groups (carboxyl group and hydroxyl group) of GW can form hydrogen bonds, whereas only one group (carboxyl group) of GA participates in the hydrogen formation in the stable structures of clusters (Figures S1-S6 of the supplementary material). Moreover, the formation Gibbs free energies of the hydrated clusters involving $\mathbf{G W}$ with relatively high population are more negative than those involving GA.

Based on this result, the hydration reaction products of oxocarboxylic acids can be ex- 

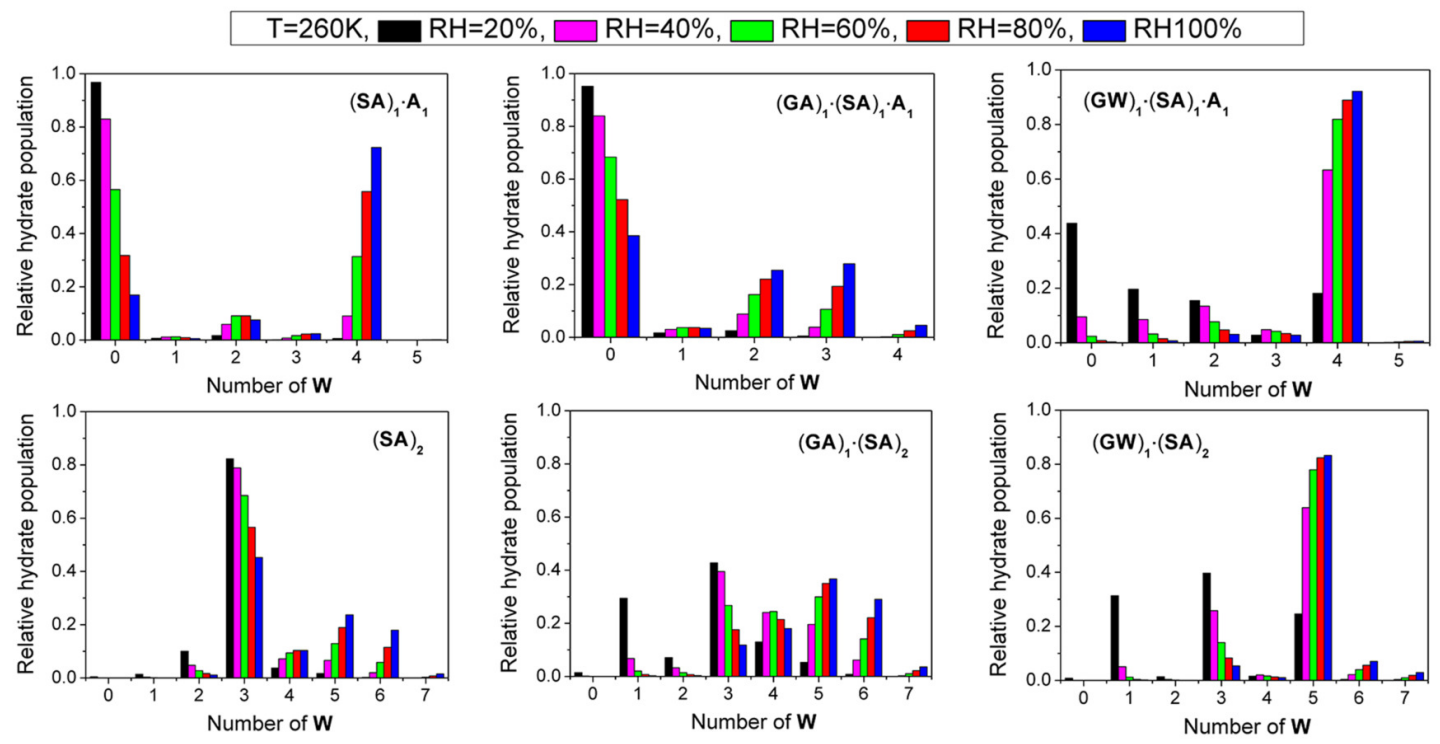

FIG. 2. Hydrate distributions of selected clusters at varying relative humidities at 260K.

pected to drastically increase the hygroscopicity of clusters.

\section{The realistic hydration reaction conversion ratio of GA-based clusters}

The hydration reaction in GA-based clusters can be uncatalyzed or catalyzed by SA, $\mathbf{W}$ or $\mathbf{A}$ as described in our previous study. ${ }^{34}$ Which kind of hydration reaction occurs in a certain GA-based cluster depends on what kinds of molecules the cluster contains. Here, we assume that the reaction is always catalyzed by the most effective (lowest activation free energy barrier) catalyst present. In addition, clusters with different number of water molecules may have different hydration reaction pathways available. For example, no hydration reaction is possible for the $(\mathbf{G A})_{1} \cdot(\mathbf{S A})_{1}$ cluster, but the $\mathbf{S A}$ catalyzed hydration reaction can occur in the $(\mathbf{G A})_{1} \cdot(\mathbf{S A})_{1} \cdot \mathbf{W}_{1}$ cluster. Therefore, two factors should be considered to calculate the realistic hydration reaction conversion ratio of GA-based clusters: one is the most effective catalysis mechanism, and the other is the relative population of the corresponding cluster. Thus, the rate constants corresponding to the catalyst with the low- 


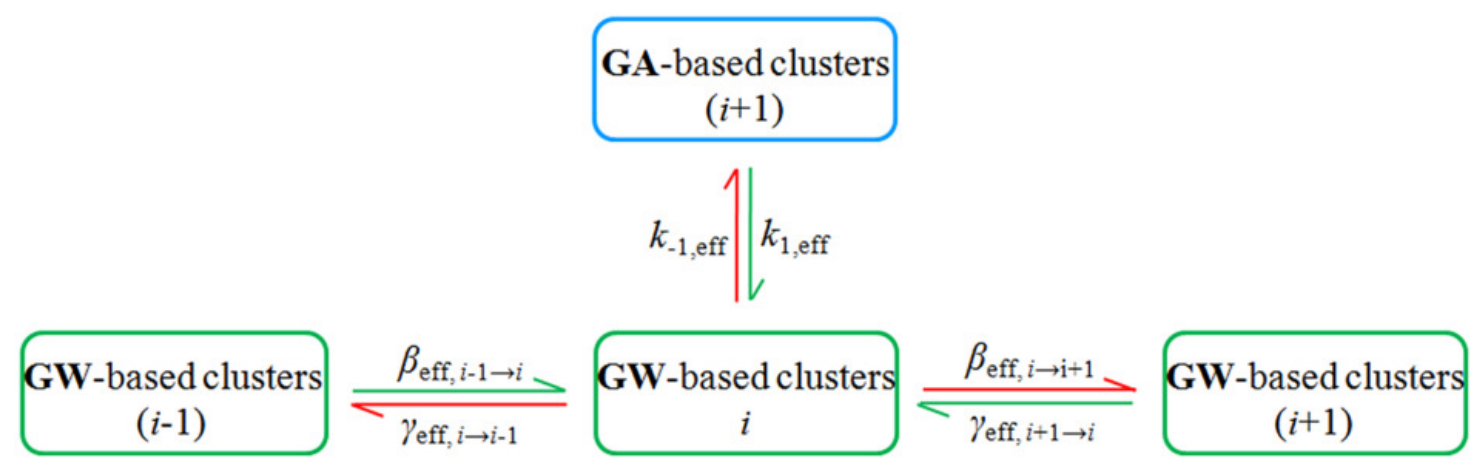

FIG. 3. The source (shown as green arrows) and sink (shown as red arrows) of $\mathbf{G W}$-based clusters. Index $i$ indicates the number of the molecules in the cluster. The hydrate distribution has been considered in the effective collisions rates $\beta_{\text {eff }}$, evaporation rates $\gamma_{\text {eff }}$ and reaction rates $k_{e f f}$. $k_{e f f}$ corresponds to the most effective catalyst for the hydration reaction, the nature of which depends on the composition of the cluster.

est activation free energy are weighted by the hydrate distribution. The $\mathbf{G W}$-based clusters with $i$ molecules in Figure 3 can be formed by the collision of the smaller $\mathbf{G W}$-based clusters containing $i$-1 molecules and the evaporation of the bigger $\mathbf{G W}$-based clusters containing $i+1$ molecules. In addition, they can be formed directly from the hydration reaction of GA-based clusters with $i+1$ molecules.

The hydration conversion ratio is one of the most important factors determining whether the hydration reaction should be considered in modelling NPF. The hydration reaction occurs either via collision of the two reactant molecules ( $\mathbf{A} \mathbf{A}$ and $\mathbf{W}$ ), or in the clusters. Depending on the cluster composition, several different catalyzed processes may be possible. ${ }^{34}$ The pathway with the lowest activation free energy barrier is always included in our process model (Figure 4).

The hydration conversion ratio $\left(X_{\mathbf{G A}}\right)$ of $\mathbf{G A}$ in the studied system is defined as

${ }_{251} X_{\mathbf{G A}}(\%)=\frac{\sum\left[(\mathbf{G W})_{1} \cdot(\mathbf{S A})_{y} \cdot \mathbf{A}_{z} \cdot \mathbf{W}_{n}\right]}{\sum\left(\left[\left[(\mathbf{G A})_{1} \cdot(\mathbf{S A})_{y} \cdot \mathbf{A}_{z} \cdot \mathbf{W}_{n}\right]+\left[(\mathbf{G W})_{1} \cdot(\mathbf{S A})_{y} \cdot \mathbf{A}_{z} \cdot \mathbf{W}_{n}\right]\right]\right)} \times 100$ 


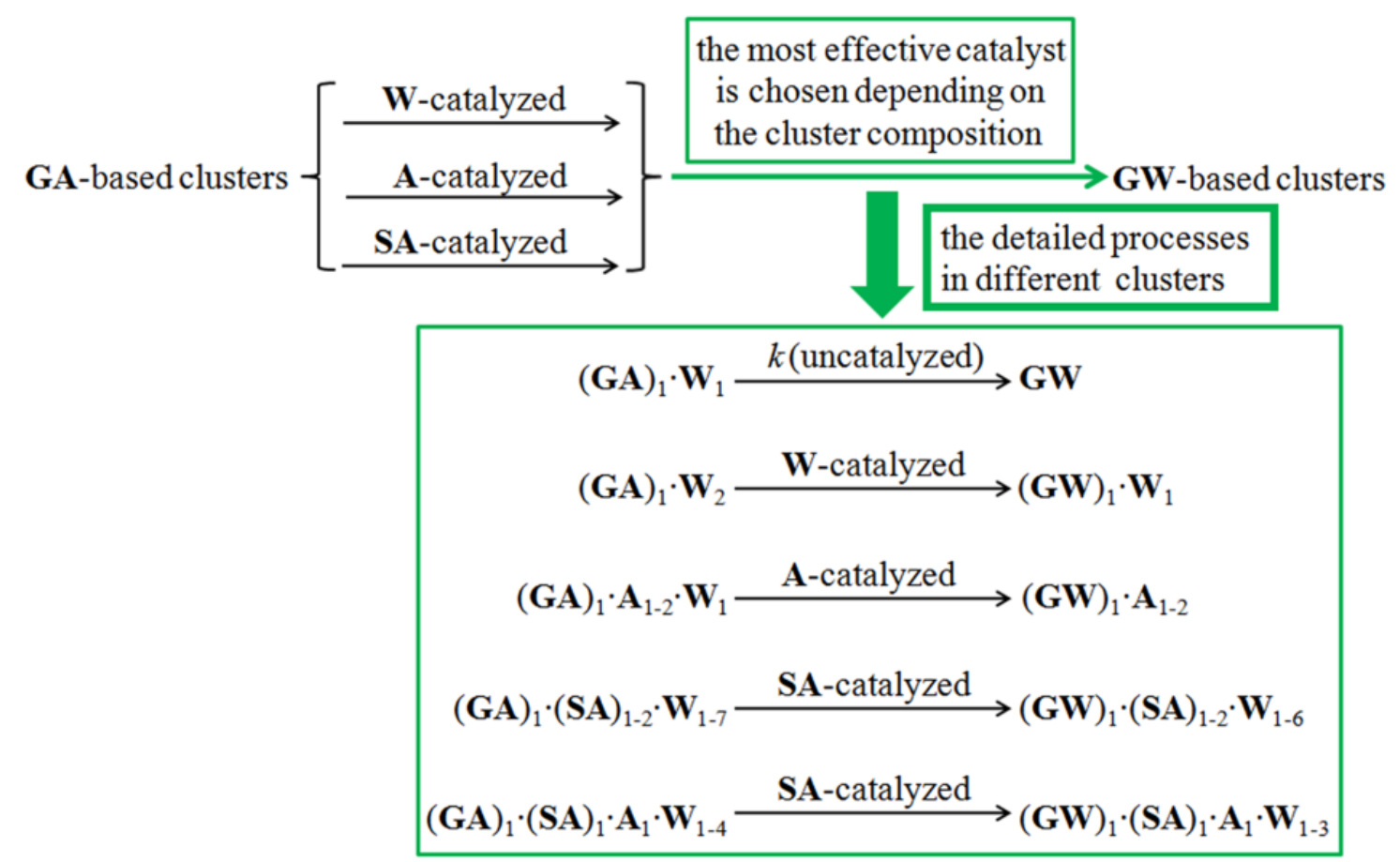

FIG. 4. Detailed information of the hydration reaction in clusters of the studied system.

where $\left[(\mathbf{G A} / \mathbf{G W})_{1} \cdot(\mathbf{S A})_{y} \cdot \mathbf{A}_{z} \cdot \mathbf{W}_{n}\right]$ is the concentration of $\mathbf{G A} / \mathbf{G W}$-based clusters (or, for $\mathrm{y}=0, \mathrm{z}=0$ and $\mathrm{n}=0, \mathbf{G A} / \mathbf{G W}$ monomers $)$. The denominator represents the sum of all $\mathbf{G A}$ or GW containing clusters (and monomers) in the system, and numerator represents the numbers of clusters where $\mathbf{G A}$ has been converted to $\mathbf{G W}$. We have modeled the conversion ratio $X_{\text {GA }}$ under different atmospheric conditions (different conditions in Figure 5 are the chosen so that they correspond to the range of values in the atmosphere). The detailed values of the conversion ratios in different conditions are given in Tables S85-S86 of the supplementary material.

Figure 5 shows that in most atmospherically relevant conditions, the ammonia (A) concentration has no effect on the conversion ratio $\left(X_{\mathbf{G A}}\right)$, while the $\mathrm{RH}$ has a moderate effect. The surprisingly weak RH dependence is caused by a "saturation" of the water-catalyzed pathway already at fairly low $\mathrm{RH}$ values. In contrast, $X_{\mathbf{G A}}$ clearly increases with decreasing temperature (Figure 5(a)), and increases with increasing sulfuric acid (SA) concentration 
(a) $[\mathrm{SA}]=10^{6},[\mathrm{~A}]=10^{9}$,

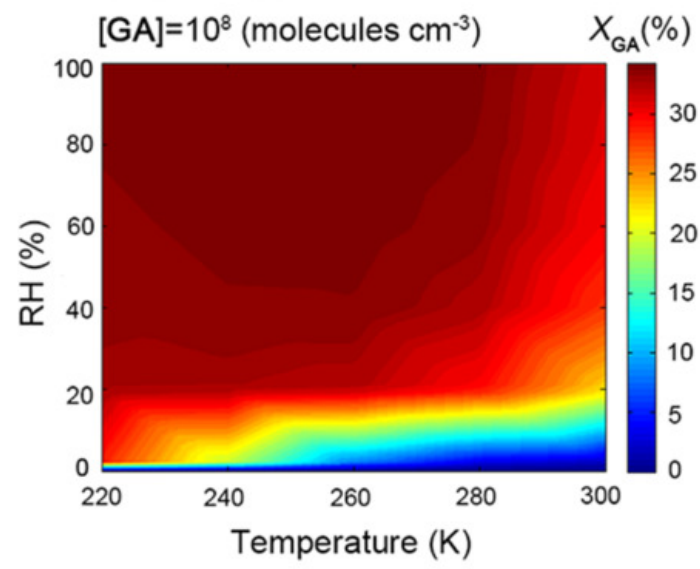

(b) $260 \mathrm{~K}, \mathrm{RH}=50 \%$,

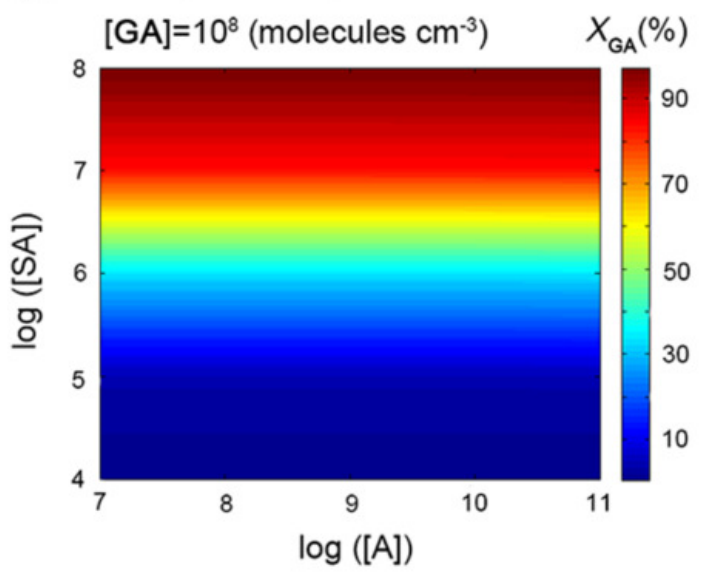

FIG. 5. The conversion ratio, $X_{\mathbf{G A}}(\%)$, of glyoxylic acid (GA) hydration forming its geminal diol (GW) at varying (a) temperatures (K) and RHs (\%) and (b) base-10 logarithm of the concentrations (molecules $\mathrm{cm}^{-3}$ ) of $\mathbf{A}$ and $\mathbf{S A}$. The color scales are shown on the right.

(Figure 5(b)). That is due to the fact that sulfuric acid catalyzes this reaction much more effectively than ammonia or water. ${ }^{34} X_{\mathbf{G A}}$ is more than $50 \%$ when $\mathbf{S A}$ concentration is more than $1.0 \times 10^{6}$ molecules $\mathrm{cm}^{-3}$, and can reach up to $85 \%$ when the concentration of SA is about $1.0 \times 10^{7}$ molecules $\mathrm{cm}^{-3}$ (Figure $5(\mathrm{~b})$ ). The relatively high conversion ratio could significantly affect the relative abundances of oxocarboxylic acids and their corresponding geminal diols and thus NPF, especially in the regions where SA is abundant, such as the polluted regions and coastal areas.

\section{Cluster formation rate}

A suitable measure for the enhancement of cluster formation rate $(J)$ by $\mathbf{G A}$ and its hydration reaction product $(\mathbf{G W})$ is the comparison of the cluster formation rate involving both GA and GW with that of SA-A-based clusters under similar conditions, i.e.

$$
r_{1}=\frac{J([\mathbf{G A}+\mathbf{G} \mathbf{W}]=x,[\mathbf{S A}]=y,[\mathbf{A}]=z)}{J([\mathbf{G A}]=0,[\mathbf{S A}]=y,[\mathbf{A}]=z)},
$$


where $r_{1}$ is the enhancement factor, $J([\mathbf{G A}+\mathbf{G} \mathbf{W}]=x],[\mathbf{S A}=y,[\mathbf{A}]=z)$ represents the formation rate of $(\mathbf{G A} / \mathbf{G W})_{x} \cdot(\mathbf{S A}){ }_{y} \mathbf{A}_{z}$ clusters with variable numbers of water molecules (and including the effect of $\mathbf{G A}$ hydration reactions $)$, and $J([\mathbf{G A}]=0,[\mathbf{S A}]=x,[\mathbf{A}]=y])$ reprensents the formation rate of the corresponding clusters without GA or GW.

As shown in Figure $6(\mathrm{a}, \mathrm{b}, \mathrm{c})$, the enhancement factor $r_{1}$ is greater than 1 , which indicates that GA can enhance the $\mathbf{S A}$-A-based cluster formation rate. $r_{1}$ increases with the increase of GA concentrations, and but only becomes significant when the temperature is lowered to $220 \mathrm{~K}$. Thus, the influence of relative humidity, sulfuric acid (SA) concentration and ammonia (A) concentration on the cluster formation rate were studied at $220 \mathrm{~K}$. The enhancement is relatively large at high $\mathrm{RH}$, low $\mathbf{S A}$ concentrations and high $\mathbf{A}$ concentrations. The enhancement factor exceeds 10 when the SA concentration lower than $1.0 \times$ $10^{6}$ molecules $\mathrm{cm}^{-3}$, and a high $\mathbf{A}$ concentration of $1.0 \times 10^{11}$ molecules $\mathrm{cm}^{-3}$, at which the absolute formation rate of GA-SA-A-based clusters is as high as $2.77 \times 10^{4} \mathrm{~cm}^{-3} \mathrm{~s}^{-1}$ (the absolute formation rates of GA-SA-A-based clusters at different temperatures, RHs and concentrations of GA, SA and A are listed in Tables S87-S89 of the supplementary material). When the SA concentration is low, and the $\mathbf{A}$ concentration high, there will be enough $\mathbf{A}$ to cluster with $\mathbf{G A}$ and $\mathbf{G W}$ despite the stronger binding between $\mathbf{S A}$ and $\mathbf{A}$ compared to GA/GW and A.

To assess the significance of the hydration reaction of $\mathbf{G A}$, we compared the cluster formation with GA present, but both with and without hydration reaction (Figure 7). A suitable measure for the effect of the hydration reaction is the ratio of formation rates in the case where the hydration reaction of $\mathbf{G A}$ to form $\mathbf{G W}$ is allowed to the rate in the case when this reaction is not occurring: 

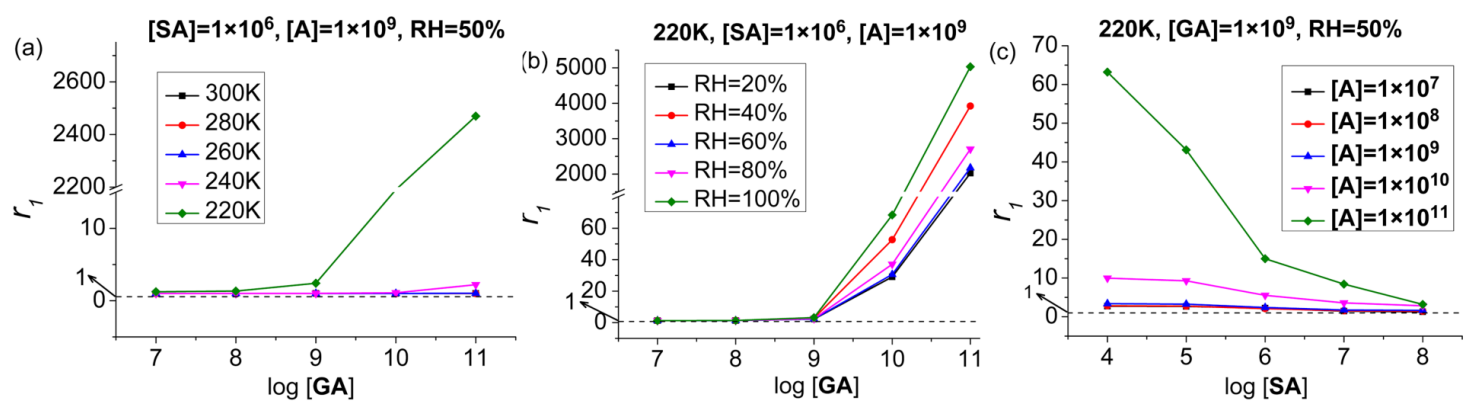

FIG. 6. Enhancement of SA-A-W cluster formation rate due to $\mathbf{G A}$ and $\mathbf{G W}$ as a function of the conditions (concentrations, molecules $\mathrm{cm}^{-3}$ ) (Eq. 14).

$$
r_{2}=\frac{J([\mathbf{G A}+\mathbf{G W}]=x,[\mathbf{S A}]=y,[\mathbf{A}]=z)}{J([\mathbf{G A}]=x,[\mathbf{S A}]=y,[\mathbf{A}]=z)},
$$

where $J([\mathbf{G A}=x,[\mathbf{S A}]=y,[\mathbf{A}]=z])$ indicates the cluster formation rate in a system involving GA but not allowing the hydration reaction.

The common trend is that $r_{2}$ increases with increasing of $\mathbf{G A}$ concentrations, but is not significant until the temperature is lowered to $220 \mathrm{~K}$ (Figure 7 (a)). Thus, the influence of relative humidity, sulfuric acid (SA) concentration and ammonia (A) concentration on the cluster formation rate were studied at $220 \mathrm{~K}$. The effect of hydration reactions on NPF is more significant at high $\mathrm{RH}$, low sulfuric acid (SA) concentration and high ammonia (A) concentration. The likely explanation for this is that when SA concentration is low and the A concentration high, there will be enough $\mathbf{A}$ for $\mathbf{G A}$ and $\mathbf{G W}$ regardless of the stronger combination between $\mathbf{S A}$ and $\mathbf{A}$. This makes the competition between $\mathbf{G A}$ and $\mathbf{G W}$ more pronounced, enhancing the ratio between cluster formation rates with hydration switched "on" and "off". Thus, both the effect of GA and its hydration reaction are most significant in cold, humid and relatively clean environments with little sulfuric acid, or agricultural regions polluted with ammonia. 

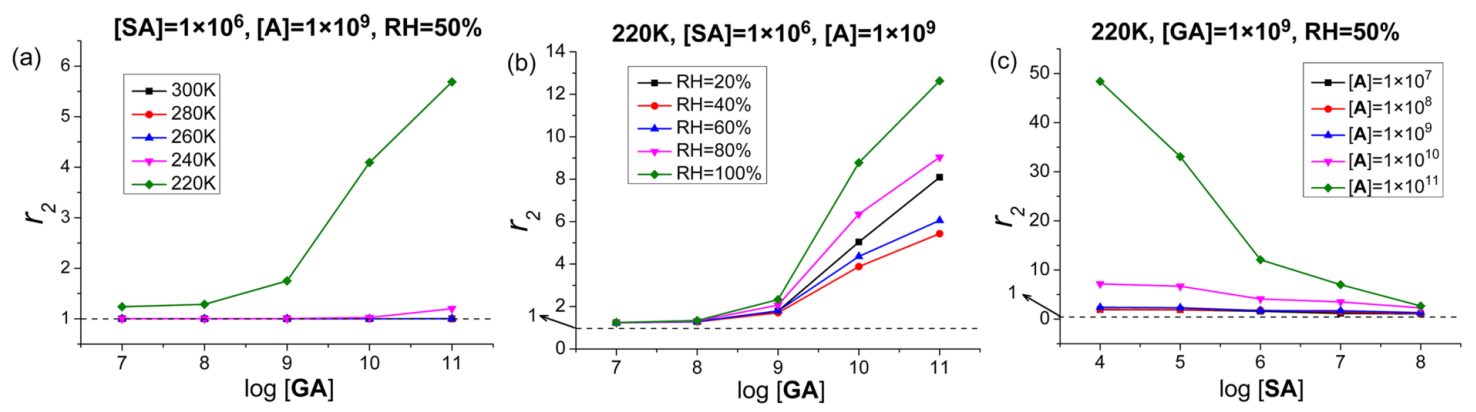

FIG. 7. Cluster formation rate involving GA and its hydration reaction relative to that involving GA but not its hydration reaction (Eq. 15).

\section{E. Cluster formation pathway}

The main cluster formation pathways involving the hydration reaction of $\mathbf{G A}$ to form GW have been further studied at 220K (Figure 8). The flux through the system proceeds principally via two clustering mechanisms: one involves pure $\mathbf{S A}$-A-clusters, and the other involves one $\mathbf{G A}$ or $\mathbf{G W}$ molecule in addition to $\mathbf{S A}$ and $\mathbf{A}$. The clusters grow out of the size region studied through the addition of sulfuric acid to $(\mathbf{S A})_{2} \cdot \mathbf{A}_{1}$ clusters. GA-based clusters easily form GW-based clusters through hydration reactions, for example converting $(\mathbf{G A})_{1} \cdot(\mathbf{S A})_{1} \cdot \mathbf{W}_{n}$ to $(\mathbf{G W})_{1} \cdot(\mathbf{S A})_{1} \cdot \mathbf{W}_{n-1}$. Though $\mathbf{G A}$ and $\mathbf{G W}$ evaporate easily from clusters from the point of view of the cluster stability, the contribution of $\mathbf{G W}$ to the formation of $(\mathbf{S A})_{2}$ or $(\mathbf{S A})_{1} \cdot \mathbf{A}_{1}$ clusters can still reach up to $77 \%$ and $100 \%$, respectively, due to the high concentration of $\mathbf{G A}$ and the high hydration conversion ratio combined with the thermodynamic stability. At the conditions corresponding to high $\mathrm{R}$ values, nearly $100 \%$ of the $(\mathbf{S A})_{2} \cdot(\mathbf{A})_{1}$ clusters are formed via $(\mathbf{G W})_{1} \cdot(\mathbf{S A})_{1} \cdot \mathbf{A}_{1}$ clusters (Figure S9 of the supplementary material). Thus, the contribution of GA to SA-A NPF is potentially of great significance especially in the regions where the hydration conversion ratio is large. 


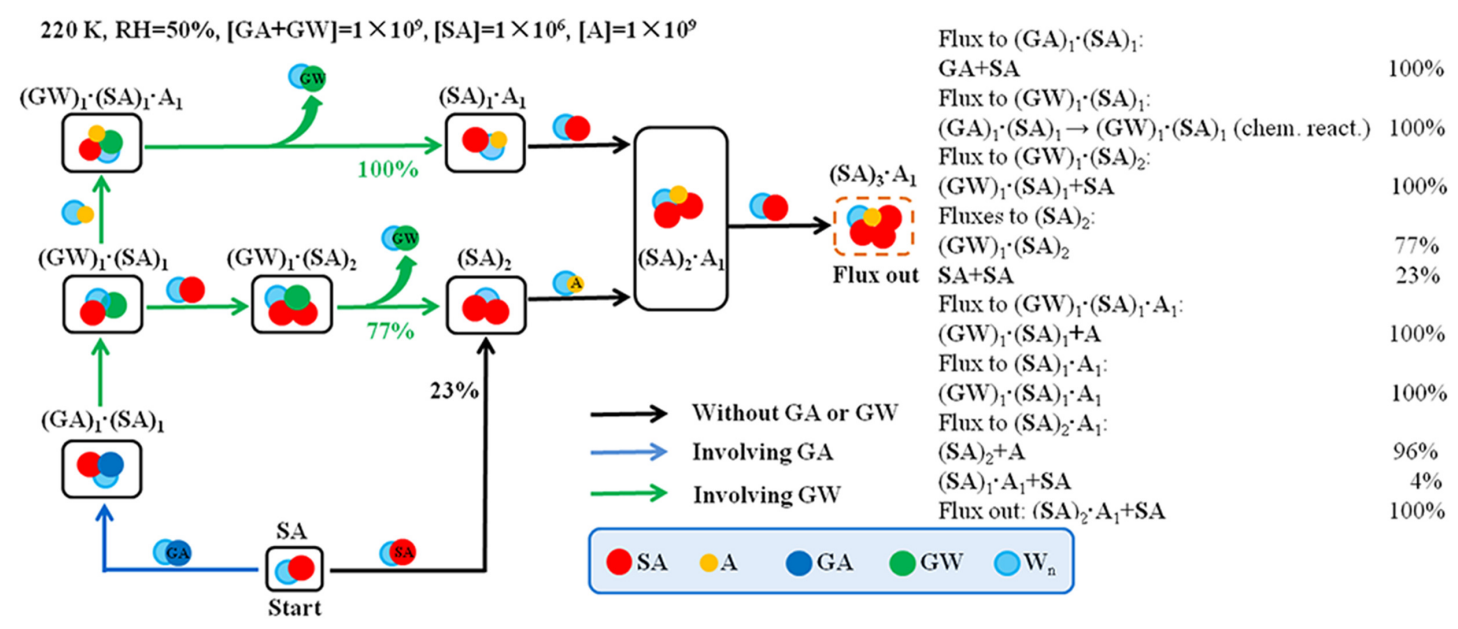

FIG. 8. Main cluster formation pathways considering the hydration reaction of $\mathbf{G A}$ forming $\mathbf{G W}$ are represented by arrows. Relative amounts of clusters formed via dominating growth pathways are indicated in the side table.

\section{CONCLUSIONS}

The clustering mechanism involving hydration reaction of glyoxylic acid, as a representative example of oxocarboxylic acids, has been studied using Density Functional Theory combined with the Atmospheric Clusters Dynamic Code. Hydration reaction induces the difference in cluster structures, and the hydration reaction products of glyoxylic acid can drastically increase the hygroscopicity of clusters. In atmospheric conditions, the total hydration reaction conversion ratio of glyoxylic acid to its product (2,2-dihydroxyacetic acid) in both gas phase and clusters can be up to $85 \%$, and the product can further participate in the clustering process. Thus, it can be speculated that the relatively high conversion ratio could significantly affect the relative abundances of oxocarboxylic acids and their corresponding geminal diols and thus NPF.

Neglecting the hydration reaction can thus induce a significant error in cluster formation rates and pathways, especially at relatively low temperature. In addition, the evaporation rates of larger oxocarboxylic acids (and especially their germinal diols) can be expected to 
be lower due to more H-bonding groups and higher molecular weights. They are thus likely to participate in the cluster formation also at higher temperatures. Thus, the hydration reaction of oxocarboxylic acids in clustering process may be of greater importance in the atmosphere. A more general inference from the present study is that the hydration reactions of oxocarboxylic acids catalyzed by clustering with sulfuric acid and ammonia can increase both the hydroscopicity and stability of clusters, and thus contribute to NPF. The present study can provide a clearer picture of the effect mechanism of oxocarboxylic acids in NPF and indicates the prospect of nucleation process involving chemical reactions, which has significant implications for the improvement of the atmospheric models.

\section{SUPPLEMENTARY MATERIAL}

See supplementary material for the the Gibbs free energy barrier (kcal/mol) and the reaction rate constants (ref. 34), the Wigner tunneling correction factor and the tunneling effect factor corrected reaction rate constants for hydration reactions of GA with W (a) uncatalyzed, (b) catalyzed by $\mathbf{W}$, (c) catalyzed by $\mathbf{S A}$ and (d) catalyzed by $\mathbf{A}$ at varying temperatures ranging from $220 \mathrm{~K}$ to $300 \mathrm{~K}$, evaporation rate coefficients of clusters, cartesian coordinates of all clusters, AIM topological parameters for the stable clusters, the realistic hydration reaction conversion ratio $\left(X_{\mathbf{G A}}\right)$, formation rates of clusters, the most stable configuration of the studied clusters, the AIM plots of the unhydrated clusters, Gibbs free energies of formation of clusters, the main cluster formation pathways considering the hydration reaction of GA forming $\mathbf{G W}$, details for the boundary conditions and complete Gaussian 09 reference (Ref. 52) 


\section{ACKNOWLEDGMENTS}

The authors thank the Chinese National Natural Science Foundation (21373025, 91544223, 21473010 and 21673018) for the support of this research. T.K. thanks the Academy of Finland for funding. H.V. thanks European Research Council (Grant 692891-DAMOCLES) and the University of Helsinki, Faculty of Science ATMATH project for funding.

\section{REFERENCES}

${ }^{1}$ K. K. Ding, X. T. Kong, J. P. Wang, L. P. Lu, W. F. Zhou, T. J. Zhan, C. L. Zhang, and S. L. Zhuang, Environ. Sci. Technol. Lett. 51, 6452 (2017).

${ }^{2}$ A. G. Rincón, M. I. Guzmán, M. R. Hoffmann, and A. J. Colussi, J. Phys. Chem. A 113, 10512 (2009).

${ }^{3}$ C. L. Heald, D. J. Jacob, R. J. Park, L. M. Russell, B. J. Huebert, J. H. Seinfeld, H. Liao, and R. J. Weber, Geophys. Res. Lett. 32, L18809 (2005).

${ }^{4}$ R. Volkamer, J. L. Jimenez, F. S. Martini, K. Dzepina, Q. Zhang, D. Salcedo, L. T. Molina, D. R. Worsnop, and M. J. Molina, Geophys. Res. Lett. 33, L17811 (2006).

${ }^{5}$ R. Y. Zhang, I. Suh, J. Zhao, D. Zhang, E. C. Fortner, X. X. Tie, L. T. Molina, and M. J. Molina, Science 304, 1487 (2004).

${ }^{6}$ R. Y. Zhang, A. Khalizov, L. Wang, M. Hu, and W. Xu, Chem. Rev. 112, 1957 (2012).

${ }^{7}$ H. L. Zhao, Q. Zhang, and L. Du, RSC Adv. 6, 71733 (2016).

${ }^{8}$ Q. Zhang and L. Du, Comput. Theor. Chem. 1078, 123 (2016).

${ }^{9}$ I. K. Ortega, N. M. Donahue, T. Kurtén, M. Kulmala, C. Focsa, and H. Vehkamäki, J. Phys. Chem. A 120, 1452 (2016).

${ }^{10}$ J. Elm, N. Myllys, T. Olenius, R. Halonen, T. Kurtén, and H. Vehkamäki, Phys. Chem. Chem. Phys. 19, 4877 (2017).

${ }^{11}$ H. B. Xie, J. Elm, R. Halonen, N. Myllys, T. Kurtén, M. Kunlmala, and H. Vehkamäki, Environ. Sci. Technol. 51, 8422 (2017).

${ }^{12}$ C. Chirs, Nature 533, 478 (2016).

${ }^{13}$ S. Li, K. Qu, H. Zhao, L. Ding, and L. Du, Chem. Phys. 472, 198 (2016). 
${ }^{14}$ H. L. Zhao, S. S. Tang, S. Y. Li, L. Ding, and L. Du, Struct. Chem 27, 1241 (2016).

${ }^{15}$ J. Elm, M. Passananti, T. Kurtén, and H. Vehkamäki, J. Phys. Chem. A 121, 6155 (2017).

${ }^{16}$ S. Guo, M. Hu, M. L. Zamora, J. F. Peng, D. J. Shang, J. Zheng, Z. F. Du, Z. J. Wu, M. Shao, L. M. Zeng, M. J. Molina, and R. Y. Zhang, Proc. Natl. Acad. Sci. U. S. A. 111, 17373 (2014).

${ }^{17}$ N. Zhao, Q. Z. Zhang, and W. X. Wang, Sci. Total Environ. 563-564, 1008 (2016).

${ }^{18}$ J. Dang, X. L. Shi, Q. Z. Zhang, and W. X. Wang, Sci. Total Environ. 517, 1 (2015).

${ }^{19}$ X. W. Wang, B. Jing, F. Tan, J. B. Ma, Y. H. Zhang, and M. F. Ge, Atmos. Chem. Phys. 17, 12797 (2017).

${ }^{20}$ V. Hirvonen, N. Myllys, T. Kurtén, and J. Elm, J. Phys. Chem. A 122, 1771 (2018).

${ }^{21}$ H. B. Xie, F. F. Ma, Y. Q., and C. J. W., J. Phys. Chem. A 121, 1657 (2017).

${ }^{22}$ J. Liu, S. Fang, Z. Wang, W. Yi, F.-M. Tao, and J. Y. Liu, Environ. Sci. Technol. 49, $13112(2015)$.

${ }^{23}$ J. J. Liu, S. Fang, W. Liu, M. Y. Wang, F. M. Tao, and J. Y. Liu, J. Phys. Chem. A 119, $102(2015)$.

${ }^{24}$ X. Shi, R. Zhang, Y. Sun, X. F., Q. Zhang, and W. Wang, Phys. Chem. Chem. Phys. 20, 1005 (2018).

${ }^{25}$ T. Kurtén, J. Elm, N. L. Prisle, K. V. Mikkelsen, C. J. Kampf, E. M. Waxman, and R. Volkamer, J. Phys. Chem. A 119, 4509 (2015).

${ }^{26}$ M. Shrivastava, C. D. Cappa, J. Fan, A. H. Goldstein, A. B. Guenther, J. L. Jimenez, C. Kuang, A. Laskin, S. T. Martin, N. L. Ng, T. Petaja, J. R. Pierce, P. J. Rasch, P. Roldin, J. H. Seinfeld, J. Shilling, J. N. Smith, J. A. Thornton, R. Volkamer, J. Wang, D. R. Worsnop, R. A. Zaveri, A. Zelenyuk, and Q. Zhang, Rev. Geophys. 55, 509 (2017).

${ }^{27}$ K. L. Plath, J. L. Axson, G. C. Nelson, K. Takahashi, R. T. Skodje, and V. Vaidaa, React. Kinet. Catal. Lett. 96, 209 (2009).

${ }^{28}$ J. L. Axson, K. Takahashi, D. O. D. Haan, and V. Vaida, Proc. Natl. Acad. Sci. U. S. A. 107, 6687 (2010).

${ }^{29}$ M. K. Hazra, J. S. Francisco, and A. Sinha, J. Phys. Chem. A 117, 11704 (2013).

${ }^{30}$ M. K. Hazra, J. S. Francisco, and A. Sinha, J. Phys. Chem. A 118, 4095 (2014).

${ }^{31}$ R. J. Weber, J. J. Marti, P. H. McMurry, F. L. Elsele, D. J. Tanner, and A. Jefferson, Chem. Eng. Commun. 151, 53 (1996).

${ }^{32}$ R. Ortiz, K. Enya, K. Sekiguchi, and K. Sakamoto, Atmos. Environ. 43, 382 (2009). 
${ }^{33}$ G. H. Wang, K. Kawamura, C. L. Cheng, J. J. Li, J. J. Cao, R. J. Zhang, T. Zhang, S. X. Liu, and Z. Z. Zhao, Environ. Sci. Technol. 46, 4783 (2012).

${ }^{34}$ L. Liu, X. H. Zhang, Z. S. Li, Y. H. Zhang, and M. F. Ge, Chemosphere 186, 430 (2017).

${ }^{35}$ M. J. McGrath, T. Olenius, I. K. Ortega, V. Loukonen, P. Paasonen, T. Kurtén, M. Kulmala, and H. Vehkamäki, Atmos. Chem. Phys. 12, 2345 (2012).

${ }^{36}$ H. Henschel, J. C. A. Navarro, T. Yli-Juuti, O. Kupiainen-Määttä, T. Olenius, I. K. Ortega, S. L. Clegg, T. Kurtén, I. Riipinen, and H. Vehkamäki, J. Phys. Chem. A 118, 2599 (2014).

${ }^{37}$ J. Zhang and M. Dolg, Phys. Chem. Chem. Phys. 17, 24173 (2015).

${ }^{38}$ J. Zhang and M. Dolg, Phys. Chem. Chem. Phys. 18, 3003 (2016).

${ }^{39}$ A. D. M. Jr., D. Bashford, M. Bellott, J. R. L. Dunbrack, J. D. Evanseck, M. J. Field, S. Fischer, J. Gao, H. Guo, S. Ha, D. Joseph-McCarthy, L. Kuchnir, K. Kuczera, F. T. K. Lau, C. Mattos, S. Michnick, T. Ngo, D. T. Nguyen, B. Prodhom, W. E. R. III, B. Roux, M. Schlenkrich, J. C. Smith, R. Stote, J. Straub, M. Watanabe, J. Wiórkiewicz-Kuczera, D. Yin, and M. Karplus, J. Phys. Chem. B 102, 3586 (1998).

${ }^{40}$ J. J. P. Stewart, J. Mol. Model. 13, 1173 (2007).

${ }^{41}$ J. J. P. Stewart, J. Mol. Model. 19, 1 (2013).

${ }^{42}$ J. J. P. Stewart, Colorado Springs, CO, USA.

${ }^{43}$ Y. Zhao and D. G. Truhlar, Theor. Chem. Acc. 120, 215 (2008).

${ }^{44}$ Y. Zhao and D. G. Truhlar, Chem. Phys. Lett. 502, 1 (2011).

${ }^{45}$ M. J. Frisch, J. A. Pople, and J. S. Binkley, Chem. Phys. Lett. 80, 3265 (1984).

${ }^{46}$ J. Elm, M. Bilde, and K. V. Mikkelsen, J. Chem. Theory Comput. 8, 2071 (2012).

${ }^{47}$ J. Elm, M. Bilde, and K. V. Mikkelsen, Phys. Chem. Chem. Phys. 15, 16442 (2013).

${ }^{48}$ J. Herb, A. B. Nadykto, and F. Q. Yu, Chem. Phys. Lett. 518, 7 (2011).

${ }^{49}$ A. B. Nadykto, F. Yu, M. V. Jakovleva, J. Herb, and Y. Xu, Entropy 13, 554 (2011).

${ }^{50}$ A. B. Nadykto, F. Yu, and J. Herb, Atmos. Chem. Phys. 9, 4031 (2009).

${ }^{51}$ J. Herb, Y. Xu, F. Yu, and A. B. Nadykto, J. Phys. Chem. A 117, 133 (2013).

${ }^{52}$ M. J. Frisch, G. W. Trucks, and H. B. S. et al., Gaussian 09, Revision A.1, Gaussian Inc. Wallingford CT (see SI for details) (2009).

${ }^{53}$ T. Lu and F. Chen, J. Comput. Chem. 33, 580 (2012).

${ }^{54}$ T. Olenius, o. Kupiainen-Määttä, I. K. Ortega, T. Kurtén, and H. Vehkamäki, J. Chem. Phys. 139, 084312 (2013). 
${ }^{55}$ L. F. Shampine and M. W. Reichelt, Siam Journal on Scientific Computing 18, 1 (1997). ${ }^{56}$ N. Bork, J. Elm, T. Olenius, and Vehkamäki, Atmos. Chem. Phys. 14, 12023 (2014).

${ }^{57}$ M. Kulmala, M. Dal Maso, M. J. M., L. Pirjola, M. Väkevä, P. Aalto, P. Miikkulainen, and C. D. Hämeri, K. O'dowd, Tellus B 53, 479490 (2001).

${ }^{58}$ J. H. Seinfel and S. N. Pandis, Atmospheric chemistry and physics: from air pollution to climate change (John Wiley \& Sons, Inc., New York, 2006).

${ }^{59}$ H. Eyring, J. Chem. Phys. 3, 107 (1935).

${ }^{60}$ M. Kumar, J. M. Anglada, and J. S. Francisco, J. Phys. Chem. A 121, 43184325 (2017).

${ }^{61}$ T. Kurtén, M. Noppel, H. Vehkamäeki, M. Salonen, and M. Kulmala, Boreal Environ. Res. 12, 431 (2007).

${ }^{62}$ I. Riipinen, S. L. Sihto, M. Kulmala, F. Arnold, M. Dal Maso, W. Birmili, K. Saarnio, K. Teinila, V. M. Kerminen, and A. Laaksonen, Atmos. Chem. Phys. 7, 1899 (2007).

${ }^{63}$ J. Almeida, S. Schobesberger, A. Kürten, I. K. Ortega, O. Kupiainen-Määttä, A. P. Praplan, A. Adamov, A. Amorim, F. Bianchi, M. Breitenlechner, A. David, J. Dommen, N. M. Donahue, A. Downard, E. Dunne, J. Duplissy, S. Ehrhart, R. C. Flagan, A. Franchin, R. Guida, J. Hakala, A. Hansel, M. Heinritzi, H. Henschel, T. Jokinen, H. Junninen, M. Kajos, J. Kangasluoma, H. Keskinen, A. Kupc, T. Kurtén, A. N. Kvashin, A. Laaksonen, K. Lehtipalo, M. Leiminger, J. Leppä, V. Loukonen, V. Makhmutov, S. Mathot, M. J. McGrath, T. Nieminen, T. Olenius, A. Onnela, T. Petäjä, F. Riccobono, I. Riipinen, M. Rissanen, L. Rondo, T. Ruuskanen, F. D. Santos, N. Sarnela, S. Schallhart, R. Schnitzhofer, J. H. Seinfeld, M. Simon, M. Sipilä, Y. Stozhkov, F. Stratmann, A. Tomé, J. Tröstl, G. Tsagkogeorgas, P. Vaattovaara, Y. Viisanen, A. Virtanen, A. Vrtala, P. E. Wagner, E. Weingartner, H. Wex, C. Williamson, D. Wimmer, P. Ye, T. Yli-Juuti, K. S. Carslaw, M. Kulmala, J. Curtius, U. Baltensperger, D. R. Worsnop, H. Vehkamäki, and J. Kirkby, Nature 502, 359 (2013).

${ }^{64}$ C. Kuang, P. H. Mcmurry, and F. L. Mccormick, Alon V.and Elsele, J. Geophys. Res. 113, D10209 (2008).

${ }^{65}$ S. Schobesberger, H. Junninen, F. Bianchi, G. Lonn, M. Ehn, K. Lehtipalo, J. Dommen, S. Ehrhart, I. K. Ortega, A. Franchin, T. Nieminena, F. Riccobono, M. Hutterli, J. Duplissy, J. Almeida, A. Amorim, M. Breitenlechner, A. J. Downard, E. M. Dunne, R. C. Flagan, M. Kajos, H. Keskinen, J. Kirkby, A. Kupc, T. K. A. Kürten, A. Laaksonen, S. Mathot, A. Onnela, A. P. Praplan, L. Rondo, F. D. Santos, S. Schallhart, 
R. Schnitzhofer, M. Sipilä, A. Tomé, H. V. G. Tsagkogeorgas, D. Wimmer, U. Baltensperger, K. S. Carslaw, J. Curtius, A. Hansel, T. Petäjä, M. Kulmala, N. M. Donahue, and D. R. Worsnop, Proc. Natl. Acad. Sci. U. S. A. 110, 17223 (2013).

${ }^{66}$ B. Graham, O. L. MayolBracero, P. Guyon, G. C. Roberts, S. Decesari, M. C. Facchini, P. Artaxo, W. Maenhaut, P. Köll, and M. O. Andreae, J. Geophys. Res.: Atmos. 107, 18047 (2002).

${ }^{67}$ W. Y. Zhao, K. Kawamura, S. Y. Yue, L. F. Wei, H. Ren, Y. Yan, M. Kang, L. J. Li, L. J. Ren, S. Lai, J. Li, Y. L. Sun, Z. F. Wang, and P. Q. Fu, Atmos. Chem. Phys. 18, 2749 (2018).

${ }^{68}$ K. Kawamura, E. Tachibana, K. Okuzawa, S. G. Aggarwal, Y. Kanaya, and Z. F. Wang, Atmos. Chem. Phys. 13, 8285 (2013).

${ }^{69}$ A. Wexler, J. Res. Natl. Bur. Stand. 80A, 775 (1976).

${ }^{70}$ U. Koch and P. L. A. Popelier, J. Phys. Chem. 99, 9747 (1995).

${ }^{71}$ U. Koch and P. L. A. Popelier, J. Phys. Org. Chem. 17, 18 (2004). 


\section{Clustering Mechanism of Oxocarboxylic Acids Involving Hydration Reaction: Implications for the Atmospheric Models}

Ling Liu, ${ }^{\text {a }}$ Oona Kupiainen-Määttä, ${ }^{\mathrm{b}}$ Haijie Zhang, ${ }^{\mathrm{a}} \mathrm{Hao} \mathrm{Li},{ }^{\mathrm{a}} \mathrm{Jie}$ Zhong ${ }^{\mathrm{c}}$, Theo Kurtén, ${ }^{\mathrm{d}}$ Hanna Vehkamäki, ${ }^{\mathrm{b}}$ Shaowen Zhang, ${ }^{\mathrm{a}}$ Yunhong Zhang, ${ }^{\mathrm{a}}$ Maofa Ge, ${ }^{\mathrm{c}}$ Xiuhui

$$
\text { Zhang, }{ }^{\mathrm{a}^{*}} \text { Zesheng } \mathrm{Li}^{\mathrm{a}^{*}}
$$

${ }^{a}$ Key Laboratory of Cluster Science, Ministry of Education of China, School of Chemistry and Chemical Engineering, Beijing Institute of Technology, Beijing 100081, China

${ }^{\mathrm{b}}$ Institute for Atmospheric and Earth System Research/Physics, University of Helsinki, PO Box 64 (Gustaf Hällströmin katu 2a), FI-00014, Helsinki, Finland

${ }^{c}$ Department of Chemistry, University of Nebraska-Lincoln, Lincoln, NE, USA 68588

${ }^{\mathrm{d}}$ Institute for Atmospheric and Earth System Research/Chemistry, University of Helsinki, PO Box 64 (Gustaf Hällströmin katu 2a), FI-00014, Helsinki, Finland

${ }^{\mathrm{e}}$ Beijing National Laboratory for Molecular Sciences (BNLMS), State Key Laboratory for Structural Chemistry of Unstable and Stable Species, Institute of Chemistry, Chinese Academy of Sciences, Beijing 100190, China

* E-mail: zhangxiuhui@bit.edu.cn (X. Zhang), zeshengli@bit.edu.cn (Z.Li). 


\section{Section 1. Tables and Figures}

Table S1. The forward and reverse Gibbs free energy barrier (kcal/mol) for hydration reactions of GA with $\mathbf{W}$ (a) uncatalyzed, (b) catalyzed by $\mathbf{W}$, (c) $\mathbf{S A}$ and (d) A relative to the corresponding pre-reactive clusters and the product clusters at varying temperatures ranging from $220 \mathrm{~K}$ to $300 \mathrm{~K}$. (Ref. 1)

Table S2. The rate constants of the forward and reverse reactions, $k_{1}$ and $k_{-1}\left(\mathrm{~s}^{-1}\right)$, respectively, for the hydration of $\mathbf{G A}$ with $\mathbf{W}$ (a) uncatalyzed, (b) catalyzed by $\mathbf{W}$, (c) SA and (d) A at varying temperatures ranging from $220 \mathrm{~K}$ to $300 \mathrm{~K}$. (Ref. 1)

Table S3. The imaginary frequencies $\left(v_{\mathrm{im}}, \mathrm{cm}^{-1}\right)$ (Ref. 1) of the transition states (TSs) and the Wigner tunneling correction factor $(\Gamma(T))$ at different temperatures $(220,240$, 260, 280 and $300 \mathrm{~K}$ ) for hydration reactions of GA with W (a) uncatalyzed, (b) catalyzed by W, (c) SA and (d) A at M06-2X/6-311++G(3df,3pd) level of theory.

Table S4. The rate constants of the forward and reverse reactions $\left(k_{1, \text { cor }}\right.$ and $\left.k_{-1, \text { cor }}\left(\mathrm{s}^{-1}\right)\right)$ corrected by the tunneling effect factor $\Gamma(\mathrm{T})$ according to Wigner at the different temperatures of $220,240,260,280$ and $300 \mathrm{~K}$.

Table S5. The evaporation rate coefficients $\left(\mathrm{s}^{-1}\right)$ of all studied clusters at varying temperatures ranging from $220 \mathrm{~K}$ to $300 \mathrm{~K}$.

Tables S6-S83. Cartesian coordinates of the studied clusters.

Table S84. AIM topological parameters for the stable clusters obtained at the M06-2X/6-311++G(3df,3pd) level (in a.u.).

Table S85. The realistic hydration reaction conversion ratio $\left(X_{\mathbf{G A}}, \%\right)$ of GA-based clusters at varying temperatures and relative humidities.

Table S86. The realistic hydration reaction conversion ratio $\left(X_{\mathbf{G A}}, \%\right)$ of GA-based clusters at varying ammonia and sulfuric acid concentrations.

Table S87. The formation rates $\left(J, \mathrm{~cm}^{-3} \mathrm{~s}^{-1}\right)$ of $(\mathbf{G A} / \mathbf{G W})_{x} \cdot(\mathbf{S A})_{y} \cdot \mathbf{A}_{z}$ clusters with the vabriations of the concentration of GA ([GA]) at different temperatures of 220, 240, 260,280 and $300 \mathrm{~K}$.

Table S88. The formation rates $\left(J, \mathrm{~cm}^{-3} \mathrm{~s}^{-1}\right)$ of $(\mathbf{G A} / \mathbf{G W})_{x} \cdot(\mathbf{S A})_{y} \cdot \mathbf{A}_{z}$ clusters with the vabriations of the concentration of GA ([GA]) at different relative humidities (RH) of $20 \%, 40 \%, 60 \%, 80 \%$ and $100 \%$. 
Table S89. The formation rates $\left(J, \mathrm{~cm}^{-3} \mathrm{~s}^{-1}\right)$ of $(\mathbf{G A} / \mathbf{G W})_{x} \cdot(\mathbf{S A})_{y} \cdot \mathbf{A}_{z}$ clusters with the vabriations of the concentration of SA ([SA]) and A ([A $])$ at $220 \mathrm{~K}$.

Figures S1-S6. Most stable configuration of $(\mathbf{G A})_{\mathbf{1}} \cdot(\mathbf{S A})_{\mathbf{1}} \cdot \mathbf{W}_{\mathbf{n}} \quad(n=0-3)$, $(\mathbf{G W})_{1} \cdot(\mathbf{S A})_{1} \cdot \mathbf{W}_{\mathbf{n}} \quad(\mathrm{n}=0-3), \quad(\mathbf{G A})_{\mathbf{1}} \cdot(\mathbf{S A})_{2} \cdot \mathbf{W}_{\mathbf{n}} \quad(\mathrm{n}=0-6), \quad(\mathbf{G W})_{\mathbf{1}} \cdot(\mathbf{S A})_{\mathbf{2}} \cdot \mathbf{W}_{\mathbf{n}} \quad(\mathrm{n}=0-7)$, $(\mathbf{G A})_{1} \cdot(\mathbf{S A})_{1} \cdot \mathbf{N}_{\mathbf{1}} \cdot \mathbf{W}_{\mathbf{n}}(\mathrm{n}=0-4)$ and $(\mathbf{G W})_{1} \cdot(\mathbf{S A})_{1} \cdot \mathbf{N}_{\mathbf{1}} \cdot \mathbf{W}_{\mathbf{n}}(\mathrm{n}=0-5)$ clusters. The lengths of the hydrogen bonds are given in $\AA$. The hydrogen bonds are shown as dashed lines.

Figure S8. Gibbs free energies ( $\mathrm{kcal} \mathrm{mol}^{-1}$ ) of formation of clusters (a) $\mathbf{O}_{1} \cdot \mathbf{S} \mathbf{A}_{1} \cdot \mathbf{W}_{\mathrm{n}}$, (b) $\mathbf{O}_{1} \cdot \mathbf{S A}_{2} \cdot \mathbf{W}_{\mathrm{n}}$ and (c) $\mathbf{O}_{1} \cdot \mathbf{S} \mathbf{A}_{1} \cdot \mathbf{N}_{1} \cdot \mathbf{W}_{\mathrm{n}}$. $\mathbf{O}$ indicates $\mathbf{G A}$ or $\mathbf{G W}$. The solid lines are to guide the eye.

Figure S9. Main cluster formation pathways considering the hydration reaction of GA forming GW are represented by arrows. Relative amounts of clusters formed via dominating growth pathways are indicated in the side table.

\section{Section 2. Boundary Conditions}

Complete Gaussian 09 reference (Reference 52)

Table S1. The forward and reverse Gibbs free energy barrier (kcal/mol) for hydration reactions of GA with $\mathbf{W}$ (a) uncatalyzed, (b) catalyzed by $\mathbf{W}$, (c) $\mathbf{S A}$ and (d) A relative to the corresponding pre-reactive clusters and the product clusters at varying temperatures ranging from $220 \mathrm{~K}$ to $300 \mathrm{~K}$. (Ref. 1)

\begin{tabular}{lccccc}
\hline Reactions & $300 \mathrm{~K}$ & $280 \mathrm{~K}$ & $260 \mathrm{~K}$ & $240 \mathrm{~K}$ & $220 \mathrm{~K}$ \\
\hline (a) forward & 38.58 & 38.33 & 38.09 & 37.85 & 37.63 \\
(a) reverse & 44.87 & 44.82 & 44.78 & 44.73 & 44.69 \\
(b) forward & 23.98 & 23.66 & 23.34 & 23.05 & 22.77 \\
(b) reverse & 30.82 & 30.68 & 30.55 & 30.43 & 30.31 \\
(c) forward & 9.50 & 9.33 & 9.17 & 9.02 & 8.88 \\
(c) reverse & 17.97 & 17.95 & 17.92 & 17.90 & 17.88 \\
(d) forward & 22.90 & 22.70 & 22.50 & 22.31 & 22.13 \\
(d) reverse & 29.77 & 29.62 & 29.48 & 29.34 & 29.21 \\
\hline
\end{tabular}


Table S2. The rate constants of the forward and reverse reactions, $k_{1}$ and $k_{-1}\left(\mathrm{~s}^{-1}\right)$, respectively, for the hydration of $\mathbf{G A}$ with $\mathbf{W}$ (a) uncatalyzed, (b) catalyzed by $\mathbf{W}$, (c) SA and (d) A at varying temperatures ranging from $220 \mathrm{~K}$ to $300 \mathrm{~K}$. The unimolecular rate constants in this table are computed relative to the reactant or product clusters (e.g. GA-W in the case of the uncatalyzed reaction).(Ref. 1)

\begin{tabular}{cllllllll}
\hline Temperatures & $k_{1}(\mathrm{a})$ & $k_{-1}(\mathrm{a})$ & $k_{1}(\mathrm{~b})$ & $k_{-1}(\mathrm{~b})$ & $k_{1}(\mathrm{c})$ & $k_{-1}(\mathrm{c})$ & $k_{1}(\mathrm{~d})$ & $k_{-1}(\mathrm{~d})$ \\
\hline 220 & $1.88 \times 1$ & $1.83 \times 1$ & $1.10 \times 1$ & $3.54 \times 1$ & $6.87 \times 1$ & $7.87 \times 1$ & $4.70 \times 1$ & $4.41 \times 1$ \\
& $0^{-25}$ & $0^{-32}$ & $0^{-10}$ & $0^{-18}$ & $0^{3}$ & $0^{-6}$ & $0^{-10}$ & $0^{-17}$ \\
240 & $1.68 \times 1$ & $9.19 \times 1$ & $5.12 \times 1$ & $9.80 \times 1$ & $3.04 \times 1$ & $2.49 \times 1$ & $2.41 \times 1$ & $9.53 \times 1$ \\
& $0^{-22}$ & $0^{-29}$ & $0^{-9}$ & $0^{-16}$ & $0^{4}$ & $0^{-4}$ & $0^{-8}$ & $0^{-15}$ \\
260 & $5.22 \times 1$ & $1.25 \times 1$ & $1.29 \times 1$ & $1.13 \times 1$ & $1.06 \times 1$ & $4.65 \times 1$ & $6.65 \times 1$ & $8.98 \times 1$ \\
& $0^{-20}$ & $0^{-25}$ & $0^{-7}$ & $0^{-13}$ & $0^{5}$ & $0^{-3}$ & $0^{-7}$ & $0^{-13}$ \\
280 & $7.03 \times 1$ & $6.03 \times 1$ & $1.99 \times 1$ & $6.58 \times 1$ & $3.05 \times 1$ & $5.73 \times 1$ & $1.12 \times 1$ & $4.40 \times 1$ \\
& $0^{-18}$ & $0^{-23}$ & $0^{-6}$ & $0^{-12}$ & $0^{5}$ & $0^{-2}$ & $0^{-5}$ & $0^{-11}$ \\
300 & $4.87 \times 1$ & $1.28 \times 1$ & $2.11 \times 10$ & $2.21 \times 1$ & $7.55 \times 1$ & $5.06 \times 1$ & $1.29 \times 1$ & $1.28 \times 1$ \\
& $0^{-16}$ & $0^{-20}$ & -5 & $0^{-10}$ & $0^{5}$ & $0^{-1}$ & $0^{-4}$ & $0^{-9}$ \\
\hline
\end{tabular}

Table S3. The imaginary frequencies $\left(v_{\mathrm{im}}, \mathrm{cm}^{-1}\right)$ (Ref. 1) of the transition states (TSs) and the Wigner tunneling correction factor $(\Gamma(T))$ at different temperatures $(220,240$, 260, 280 and $300 \mathrm{~K}$ ) for hydration reactions of GA with W (a) uncatalyzed, (b) catalyzed by $\mathbf{W}$, (c) SA and (d) A at M06-2X/6-311++G(3df,3pd) level of theory.

\begin{tabular}{cllllllll}
\hline Temperatures & $v_{\text {im }}(\mathrm{a})$ & $\Gamma(T)(\mathrm{a})$ & $v_{\mathrm{im}}(\mathrm{b})$ & $\Gamma(T)(\mathrm{b})$ & $v_{\text {im }}(\mathrm{c})$ & $\Gamma(T)(\mathrm{c})$ & $v_{\text {im }}(\mathrm{d})$ & $\Gamma(T)(\mathrm{d})$ \\
\hline 220 & 1368 & 4.34 & 827 & 2.22 & 277 & 1.14 & 558 & 1.56 \\
240 & & 3.81 & & 2.02 & & 1.12 & & 1.48 \\
260 & & 3.39 & & 1.87 & & 1.10 & & 1.40 \\
280 & 3.06 & & 1.75 & & 1.08 & & 1.34 \\
300 & & 2.80 & & 1.66 & & 1.07 & & 1.30 \\
\hline
\end{tabular}


Table S4. The rate constants of the forward and reverse reactions $\left(k_{1, \text { cor }}\right.$ and $\left.k_{-1, \text { cor }}\left(\mathrm{s}^{-1}\right)\right)$ corrected by the tunneling effect factor $\Gamma(T)$ according to Wigner for the hydration reactions of GA with $\mathbf{W}$ (a) uncatalyzed, (b) catalyzed by $\mathbf{W}$, (c) $\mathbf{S A}$ and (d) $\mathbf{A}$ at the different temperatures of 220, 240, 260, 280 and $300 \mathrm{~K}$.

\begin{tabular}{cllllllll}
\hline Temperatures & $k_{1, \text { cor }}(\mathrm{a})$ & $k_{-1, \text { cor }}(\mathrm{a})$ & $k_{1, \text { cor }}(\mathrm{b})$ & $k_{-1, \text { cor }(\mathrm{b})}$ & $k_{1, \text { cor }}(\mathrm{c})$ & $k_{-1, \text { cor }}(\mathrm{c})$ & $k_{1, \text { cor }}(\mathrm{d})$ & $k_{-1, \text { cor }}(\mathrm{d})$ \\
\hline 220 & $8.16 \times 10^{-}$ & $7.94 \times 10^{-}$ & $2.44 \times 10^{-}$ & $7.86 \times 10^{-}$ & $7.81 \times 10$ & $8.95 \times 10^{-}$ & $1.05 \times 10^{-}$ & $9.86 \times 10^{-}$ \\
& 25 & 32 & 10 & 18 & 3 & 6 & 9 & 17 \\
240 & $6.40 \times 10^{-}$ & $3.50 \times 10^{-}$ & $1.04 \times 10^{-}$ & $1.99 \times 10^{-}$ & $3.39 \times 10$ & $2.78 \times 10^{-}$ & $4.91 \times 10^{-}$ & $1.94 \times 10^{-}$ \\
& 22 & 28 & 8 & 15 & 4 & 4 & 8 & 14 \\
260 & $1.77 \times 10^{-}$ & $4.24 \times 10^{-}$ & $2.42 \times 10^{-}$ & $2.12 \times 10^{-}$ & $1.16 \times 10$ & $5.11 \times 10^{-}$ & $1.25 \times 10^{-}$ & $1.69 \times 10^{-}$ \\
& 19 & 25 & 7 & 13 & 5 & 3 & 6 & 12 \\
280 & $2.15 \times 10^{-}$ & $1.85 \times 10^{-}$ & $3.49 \times 10^{-}$ & $1.15 \times 10^{-}$ & $3.31 \times 10$ & $6.21 \times 10^{-}$ & $1.98 \times 10^{-}$ & $7.75 \times 10^{-}$ \\
& 17 & 22 & 6 & 11 & 5 & 2 & 5 & 11 \\
300 & $1.36 \times 10^{-}$ & $3.58 \times 10^{-}$ & $3.50 \times 10^{-}$ & $3.66 \times 10^{-}$ & $8.11 \times 10$ & $5.43 \times 10^{-}$ & $2.15 \times 10^{-}$ & $2.13 \times 10^{-}$ \\
& 15 & 20 & 5 & 10 & 5 & 1 & 4 & 9 \\
\hline
\end{tabular}

Table S5. The evaporation rate coefficients $\left(\mathrm{s}^{-1}\right)$ of all studied clusters at varying temperatures ranging from $220 \mathrm{~K}$ to $300 \mathrm{~K}$.

\begin{tabular}{lccccc}
\hline Clusters & $300 \mathrm{~K}$ & $280 \mathrm{~K}$ & $260 \mathrm{~K}$ & $240 \mathrm{~K}$ & $220 \mathrm{~K}$ \\
\hline$(\mathbf{S A})_{2} \rightarrow \mathbf{S A}+\mathbf{S A}$ & $1.3 \mathrm{E}+03$ & $1.5 \mathrm{E}+02$ & $1.2 \mathrm{E}+01$ & $0.6 \mathrm{E}+00$ & $4.0 \mathrm{E}-02$ \\
$(\mathbf{S A})_{1} \mathbf{A}_{1} \rightarrow \mathbf{A}+\mathbf{S A}$ & $3.4 \mathrm{E}+04$ & $5.0 \mathrm{E}+03$ & $5.4 \mathrm{E}+02$ & $4.1 \mathrm{E}+01$ & $1.9 \mathrm{E}+00$ \\
$(\mathbf{S A})_{2} \mathbf{A}_{1} \rightarrow(\mathbf{S A})_{1} \mathbf{A}_{1}+\mathbf{S A}$ & $3.1 \mathrm{E}+00$ & $1.3 \mathrm{E}-01$ & $3.3 \mathrm{E}-03$ & $4.5 \mathrm{E}-05$ & $2.8 \mathrm{E}-07$ \\
$(\mathbf{S A})_{2} \mathbf{A}_{1} \rightarrow \mathbf{A}+(\mathbf{S A})_{2}$ & $5.5 \mathrm{E}+01$ & $2.8 \mathrm{E}+00$ & $9.7 \mathrm{E}-02$ & $1.9 \mathrm{E}-03$ & $1.7 \mathrm{E}-05$ \\
$(\mathbf{G A})_{1}(\mathbf{S A})_{1} \rightarrow \mathbf{G A}+\mathbf{S A}$ & $2.7 \mathrm{E}+05$ & $3.6 \mathrm{E}+04$ & $3.6 \mathrm{E}+03$ & $2.4 \mathrm{E}+02$ & $9.9 \mathrm{E}+00$ \\
$\left.(\mathbf{G A})_{1} \mathbf{S A}\right)_{2} \rightarrow(\mathbf{G A})_{1}(\mathbf{S A})_{1}+\mathbf{S A}$ & $8.1 \mathrm{E}+04$ & $8.0 \mathrm{E}+03$ & $5.6 \mathrm{E}+02$ & $2.6 \mathrm{E}+01$ & $6.5 \mathrm{E}-01$ \\
$(\mathbf{G A})_{1}(\mathbf{S A})_{2} \rightarrow(\mathbf{S A})_{2}+\mathbf{G A}$ & $6.9 \mathrm{E}+06$ & $8.0 \mathrm{E}+05$ & $6.8 \mathrm{E}+04$ & $4.0 \mathrm{E}+03$ & $1.3 \mathrm{E}+02$ \\
$(\mathbf{G A})_{1} \mathbf{A}_{1} \rightarrow \mathbf{G A}+\mathbf{A}$ & $1.4 \mathrm{E}+08$ & $3.3 \mathrm{E}+07$ & $6.6 \mathrm{E}+06$ & $9.6 \mathrm{E}+05$ & $1.8 \mathrm{E}+06$ \\
$(\mathbf{G A})_{1}(\mathbf{S A})_{1} \mathbf{A}_{1} \rightarrow(\mathbf{G A})_{1} \mathbf{A}_{1}+\mathbf{S A}$ & $7.0 \mathrm{E}+01$ & $5.3 \mathrm{E}+00$ & $2.6 \mathrm{E}-01$ & $8.1 \mathrm{E}-03$ & $7.2 \mathrm{E}-06$ \\
\hline
\end{tabular}




\begin{tabular}{lccccc}
\hline$(\mathbf{G A})_{1}(\mathbf{S A})_{1} \mathbf{A}_{1} \rightarrow(\mathbf{G A})_{1}(\mathbf{S A})_{1}+\mathbf{A}$ & $5.2 \mathrm{E}+04$ & $7.1 \mathrm{E}+03$ & $7.0 \mathrm{E}+02$ & $4.7 \mathrm{E}+01$ & $1.9 \mathrm{E}+00$ \\
$(\mathbf{G A})_{1}(\mathbf{S A})_{1} \mathbf{A}_{1} \rightarrow(\mathbf{S A})_{1} \mathbf{A}_{1}+\mathbf{G A}$ & $2.5 \mathrm{E}+05$ & $3.2 \mathrm{E}+04$ & $2.9 \mathrm{E}+03$ & $1.7 \mathrm{E}+02$ & $6.0 \mathrm{E}+00$ \\
$(\mathbf{G W})_{1}(\mathbf{S A})_{1} \rightarrow \mathbf{G W}+\mathbf{S A}$ & $7.7 \mathrm{E}+05$ & $9.6 \mathrm{E}+04$ & $8.8 \mathrm{E}+03$ & $5.3 \mathrm{E}+02$ & $1.9 \mathrm{E}+01$ \\
$(\mathbf{G W})_{1}(\mathbf{S A})_{2} \rightarrow(\mathbf{G W})_{1}(\mathbf{S A})_{1}+\mathbf{S A}$ & $7.2 \mathrm{E}+04$ & $7.3 \mathrm{E}+03$ & $5.1 \mathrm{E}+02$ & $2.3 \mathrm{E}+01$ & $6.0 \mathrm{E}-01$ \\
$(\mathbf{G W})_{1}(\mathbf{S A})_{2} \rightarrow(\mathbf{G W})_{1}+(\mathbf{S A})_{2}$ & $2.1 \mathrm{E}+07$ & $2.3 \mathrm{E}+06$ & $1.8 \mathrm{E}+05$ & $9.7 \mathrm{E}+03$ & $2.8 \mathrm{E}+02$ \\
$(\mathbf{G W})_{1} \mathbf{A} \rightarrow \rightarrow \mathbf{G W}+\mathbf{A}$ & $5.5 \mathrm{E}+08$ & $1.4 \mathrm{E}+08$ & $3.0 \mathrm{E}+07$ & $4.7 \mathrm{E}+06$ & $1.1 \mathrm{E}+05$ \\
$(\mathbf{G W})_{1}(\mathbf{S A})_{1} \mathbf{A}_{1} \rightarrow(\mathbf{G W})_{1} \mathbf{A} \mathbf{A}_{1}+\mathbf{S A}$ & $1.5 \mathrm{E}+02$ & $8.4 \mathrm{E}+00$ & $3.2 \mathrm{E}-01$ & $6.8 \mathrm{E}-03$ & $3.4 \mathrm{E}-04$ \\
$(\mathbf{G W})_{1}(\mathbf{S A})_{1} \mathbf{A}_{1} \rightarrow(\mathbf{G W})_{1}(\mathbf{S A})_{1}+\mathbf{A}$ & $1.1 \mathrm{E}+05$ & $1.3 \mathrm{E}+04$ & $1.1 \mathrm{E}+03$ & $6.2 \mathrm{E}+01$ & $2.0 \mathrm{E}+00$ \\
$(\mathbf{G W})_{1}(\mathbf{S A})_{1} \mathbf{A}_{1} \rightarrow \mathbf{G W}+(\mathbf{S A})_{1} \mathbf{A}_{1}$ & $1.8 \mathrm{E}+06$ & $1.9 \mathrm{E}+05$ & $1.3 \mathrm{E}+04$ & $6.0 \mathrm{E}+02$ & $1.5 \mathrm{E}+01$ \\
\hline
\end{tabular}

Table S6. Cartesian coordinate of SA.

\begin{tabular}{|c|c|c|c|}
\hline Atoms & $\mathrm{X}$ & $\mathrm{Y}$ & $\mathrm{Z}$ \\
\hline $\mathrm{S}$ & 0.000000 & 0.00000000 & 0.15432300 \\
$\mathrm{O}$ & 0.000000 & 1.24571500 & 0.82033500 \\
$\mathrm{O}$ & 0.000000 & -1.24571500 & 0.82033500 \\
$\mathrm{O}$ & 1.223691 & -0.04024200 & -0.83614500 \\
$\mathrm{O}$ & -1.223691 & 0.04024200 & -0.83614500 \\
$\mathrm{H}$ & -1.452033 & -0.85852000 & -1.10809700 \\
$\mathrm{H}$ & 1.452033 & 0.85852000 & -1.10809700 \\
\hline
\end{tabular}

Table S7. Cartesian coordinate of $(\mathbf{S A})_{1} \mathbf{W}_{1}$.

\begin{tabular}{|c|c|c|c|}
\hline Atoms & $\mathrm{X}$ & $\mathrm{Y}$ & $\mathrm{Z}$ \\
\hline $\mathrm{S}$ & 0.574277 & -0.075955 & 0.121591 \\
$\mathrm{O}$ & -0.215605 & 0.288808 & 1.248460 \\
$\mathrm{O}$ & 1.737700 & -0.870444 & 0.247437 \\
$\mathrm{O}$ & -0.338533 & -0.732296 & -0.941762 \\
$\mathrm{O}$ & 0.979121 & 1.291265 & -0.552739 \\
$\mathrm{H}$ & -1.289890 & -0.469017 & -0.773800 \\
$\mathrm{H}$ & 1.736148 & 1.150356 & -1.135650 \\
\hline
\end{tabular}




\begin{tabular}{|l|l|l|l|}
\hline $\mathrm{O}$ & -2.661959 & 0.105985 & -0.109758 \\
$\mathrm{H}$ & -2.259274 & 0.361340 & 0.731812 \\
$\mathrm{H}$ & -3.381199 & -0.493949 & 0.099086 \\
\hline
\end{tabular}

Table S8. Cartesian coordinate of $(\mathbf{S A})_{1} \mathbf{W}_{2}$.

\begin{tabular}{|c|c|c|c|}
\hline Atoms & $\mathrm{X}$ & $\mathrm{Y}$ & $\mathrm{Z}$ \\
\hline $\mathrm{S}$ & 0.986992 & -0.150388 & -0.099481 \\
$\mathrm{O}$ & 0.432583 & 1.170078 & -0.640315 \\
$\mathrm{O}$ & 1.134685 & 0.126299 & 1.452466 \\
$\mathrm{O}$ & -0.005244 & -1.171702 & -0.181044 \\
$\mathrm{O}$ & 2.277203 & -0.349376 & -0.644188 \\
$\mathrm{H}$ & -0.562404 & 1.301754 & -0.403772 \\
$\mathrm{H}$ & 1.971276 & 0.580502 & 1.615265 \\
$\mathrm{H}$ & -2.423091 & 0.553179 & -0.021326 \\
$\mathrm{O}$ & -2.007743 & 1.446196 & -0.076036 \\
$\mathrm{H}$ & -2.204764 & 1.896542 & 0.747917 \\
$\mathrm{H}$ & -1.846181 & -1.395479 & 0.029141 \\
$\mathrm{O}$ & -2.764155 & -1.093281 & 0.112361 \\
$\mathrm{H}$ & -3.265331 & -1.555999 & -0.561485 \\
\hline
\end{tabular}

Table S9. Cartesian coordinate of $(\mathbf{S A})_{1} \mathbf{W}_{3}$.

\begin{tabular}{|c|c|c|c|}
\hline Atoms & $\mathrm{X}$ & $\mathrm{Y}$ & $\mathrm{Z}$ \\
\hline $\mathrm{O}$ & -1.506990 & -1.371735 & -1.163177 \\
$\mathrm{H}$ & -1.773950 & -0.567008 & -1.621186 \\
$\mathrm{H}$ & -1.971831 & -1.328300 & -0.314030 \\
$\mathrm{~S}$ & 1.244190 & 0.029228 & 0.161933 \\
$\mathrm{O}$ & 2.620912 & 0.184108 & 0.414141 \\
$\mathrm{O}$ & 1.056128 & -1.232150 & -0.708744 \\
$\mathrm{O}$ & 0.784181 & 1.192212 & -0.765548 \\
\hline
\end{tabular}




\begin{tabular}{|l|l|l|l|}
\hline $\mathrm{O}$ & 0.322056 & -0.028098 & 1.266286 \\
$\mathrm{H}$ & 0.082261 & -1.372103 & -0.911487 \\
$\mathrm{H}$ & -0.192657 & 1.341878 & -0.695079 \\
$\mathrm{O}$ & -1.876525 & 1.466547 & -0.654087 \\
$\mathrm{H}$ & -2.225041 & 1.049168 & 0.154071 \\
$\mathrm{H}$ & -2.243910 & 2.351570 & -0.702420 \\
$\mathrm{O}$ & -2.325355 & -0.349927 & 1.336061 \\
$\mathrm{H}$ & -2.804143 & -0.522403 & 2.148080 \\
$\mathrm{H}$ & -1.373038 & -0.308106 & 1.551677 \\
\hline
\end{tabular}

Table S10. Cartesian coordinate of $(\mathbf{S A})_{1} \mathbf{W}_{4}$.

\begin{tabular}{|c|c|c|c|}
\hline Atoms & $\mathrm{X}$ & $\mathrm{Y}$ & $\mathrm{Z}$ \\
\hline $\mathrm{S}$ & 1.123487 & -0.004287 & -0.013165 \\
$\mathrm{O}$ & 0.739603 & -1.272553 & 0.590085 \\
$\mathrm{O}$ & 0.706005 & 0.133641 & -1.391936 \\
$\mathrm{O}$ & 2.705172 & -0.008742 & -0.115936 \\
$\mathrm{O}$ & 0.761365 & 1.128796 & 0.824541 \\
$\mathrm{H}$ & 3.083838 & -0.065877 & 0.769689 \\
$\mathrm{H}$ & -0.467606 & 2.096555 & -0.086629 \\
$\mathrm{O}$ & -1.312734 & -1.687785 & -1.555681 \\
$\mathrm{H}$ & -0.980622 & -2.425085 & -1.032924 \\
$\mathrm{H}$ & -0.510192 & -1.161006 & -1.743361 \\
$\mathrm{O}$ & -2.367127 & 0.028387 & -0.010740 \\
$\mathrm{H}$ & -2.009490 & -0.700950 & -0.642459 \\
$\mathrm{H}$ & -2.007591 & -0.175284 & 0.931298 \\
$\mathrm{O}$ & -1.303753 & -0.538247 & 2.221235 \\
$\mathrm{H}$ & -0.985665 & 0.265340 & 2.644566 \\
$\mathrm{H}$ & -0.496223 & -0.931638 & 1.828969 \\
$\mathrm{O}$ & -1.265682 & 2.224447 & -0.638203 \\
\hline & & & \\
\hline & & & \\
\hline
\end{tabular}




\begin{tabular}{|l|l|l|l|}
\hline$H$ & -0.927870 & 2.167923 & -1.538213 \\
$H$ & -1.977167 & 0.935061 & -0.307204 \\
\hline
\end{tabular}

Table S11. Cartesian coordinate of $(\mathbf{S A})_{1} \mathbf{W}_{5}$.

\begin{tabular}{|c|c|c|c|}
\hline Atoms & $x$ & $Y$ & Z \\
\hline$S$ & -0.273434 & -0.246988 & -0.622495 \\
\hline 0 & -0.398343 & 1.167897 & -0.272211 \\
\hline 0 & 0.858168 & -0.497366 & -1.519686 \\
\hline $\mathrm{O}$ & -0.235248 & -1.082549 & 0.570541 \\
\hline 0 & -1.500468 & -0.644089 & -1.461085 \\
\hline $\mathrm{H}$ & 1.097808 & 2.037633 & 0.038707 \\
\hline 0 & -3.592423 & -0.119444 & -0.067986 \\
\hline $\mathrm{H}$ & -3.200360 & 0.217815 & 0.769273 \\
\hline $\mathrm{H}$ & -2.360318 & -0.453730 & -0.954804 \\
\hline $\mathrm{H}$ & -4.166899 & 0.571888 & -0.402824 \\
\hline $\mathrm{H}$ & 2.233097 & 3.046937 & -0.317646 \\
\hline 0 & 2.033085 & 2.275923 & 0.216811 \\
\hline $\mathrm{H}$ & 2.774329 & 0.930368 & -0.062445 \\
\hline $\mathrm{H}$ & 2.850674 & -0.646137 & 0.453583 \\
\hline 0 & 2.973273 & -0.024198 & -0.342109 \\
\hline $\mathrm{H}$ & 2.137529 & -0.257546 & -0.941678 \\
\hline $\mathrm{H}$ & -1.612224 & -0.098600 & 2.178047 \\
\hline 0 & -2.087523 & 0.721596 & 2.004566 \\
\hline $\mathrm{H}$ & -1.477711 & 1.191145 & 1.416651 \\
\hline $\mathrm{H}$ & 2.483859 & -2.537246 & 1.475842 \\
\hline 0 & 2.240301 & -1.609699 & 1.501551 \\
\hline $\mathrm{H}$ & 1.288589 & -1.555293 & 1.264080 \\
\hline
\end{tabular}

Table S12. Cartesian coordinate of A. 


\begin{tabular}{|c|c|c|c|}
\hline Atoms & $X$ & $Y$ & $Z$ \\
\hline N & 0.000000 & 0.113488 & 0.000000 \\
$H$ & -0.939049 & -0.264998 & 0.000000 \\
$H$ & 0.469524 & -0.264710 & 0.813353 \\
$H$ & 0.469524 & -0.264710 & -0.813353 \\
\hline
\end{tabular}

Table S13. Cartesian coordinate of $\mathbf{A}_{1} \mathbf{W}_{1}$.

\begin{tabular}{|c|c|c|c|}
\hline Atoms & $\mathrm{X}$ & $\mathrm{Y}$ & $\mathrm{Z}$ \\
\hline $\mathrm{N}$ & 1.379382 & 0.022191 & -0.000290 \\
$\mathrm{H}$ & 1.906343 & 0.877370 & -0.125814 \\
$\mathrm{H}$ & 1.651266 & -0.610741 & -0.742711 \\
$\mathrm{H}$ & 1.681236 & -0.392918 & 0.872774 \\
$\mathrm{O}$ & -1.546280 & -0.104761 & -0.000013 \\
$\mathrm{H}$ & -1.938012 & 0.768906 & -0.000235 \\
$\mathrm{H}$ & -0.586266 & 0.040132 & -0.001883 \\
\hline
\end{tabular}

Table S14. Cartesian coordinate of $\mathbf{A}_{1} \mathbf{W}_{2}$.

\begin{tabular}{|c|c|c|c|}
\hline Atoms & $\mathrm{X}$ & $\mathrm{Y}$ & $\mathrm{Z}$ \\
\hline $\mathrm{N}$ & 1.592765 & 0.547711 & -0.000357 \\
$\mathrm{H}$ & 2.123148 & 0.822936 & 0.816595 \\
$\mathrm{H}$ & 0.774022 & 1.149874 & -0.055052 \\
$\mathrm{H}$ & 2.169493 & 0.731535 & -0.811561 \\
$\mathrm{O}$ & -0.261677 & -1.561856 & -0.096764 \\
$\mathrm{H}$ & -0.230616 & -2.187599 & 0.627884 \\
$\mathrm{H}$ & 0.546715 & -1.012136 & -0.023082 \\
$\mathrm{O}$ & -1.377614 & 0.993459 & 0.076976 \\
$\mathrm{H}$ & -2.180150 & 1.174600 & -0.412842 \\
$\mathrm{H}$ & -1.237645 & 0.033985 & 0.018858 \\
\hline
\end{tabular}


Table S15. Cartesian coordinate of $\mathbf{W}$.

\begin{tabular}{|c|c|c|c|}
\hline Atoms & $X$ & $Y$ & $Z$ \\
\hline O & 0.000000 & 0.000000 & 0.116638 \\
H & 0.000000 & 0.760974 & -0.466554 \\
H & 0.000000 & -0.760974 & -0.466554 \\
\hline
\end{tabular}

Table S16. Cartesian coordinate of GA.

\begin{tabular}{|c|c|c|c|}
\hline Atoms & $\mathrm{X}$ & $\mathrm{Y}$ & $\mathrm{Z}$ \\
\hline $\mathrm{C}$ & 0.937401 & -0.551457 & 0.000003 \\
$\mathrm{O}$ & 1.853227 & 0.206905 & -0.000003 \\
$\mathrm{H}$ & 1.042330 & -1.647548 & 0.000010 \\
$\mathrm{C}$ & -0.527858 & -0.103639 & 0.000002 \\
$\mathrm{O}$ & -1.418093 & -0.901561 & -0.000003 \\
$\mathrm{O}$ & -0.670546 & 1.215069 & 0.000001 \\
$\mathrm{H}$ & -1.616295 & 1.414820 & 0.000001 \\
\hline
\end{tabular}

Table S17. Cartesian coordinate of $(\mathbf{G A})_{1} \mathbf{W}_{1}$.

\begin{tabular}{|c|c|c|c|}
\hline Atoms & $\mathrm{X}$ & $\mathrm{Y}$ & $\mathrm{Z}$ \\
\hline $\mathrm{C}$ & -1.773556 & 0.371946 & 0.002672 \\
$\mathrm{O}$ & -2.503808 & -0.566636 & 0.014299 \\
$\mathrm{H}$ & -2.116451 & 1.418692 & -0.002761 \\
$\mathrm{C}$ & -0.245099 & 0.252841 & -0.003052 \\
$\mathrm{O}$ & 0.428977 & 1.254323 & 0.001637 \\
$\mathrm{O}$ & 0.194631 & -0.979595 & -0.010335 \\
$\mathrm{H}$ & 1.181337 & -0.959835 & -0.011526 \\
$\mathrm{O}$ & 2.776772 & -0.269251 & -0.076082 \\
$\mathrm{H}$ & 3.465216 & -0.341379 & 0.587477 \\
$\mathrm{H}$ & 2.409256 & 0.623067 & -0.007067 \\
\hline
\end{tabular}


Table S18. Cartesian coordinate of $(\mathbf{G A})_{1} \mathbf{W}_{2}$.

\begin{tabular}{|c|c|c|c|}
\hline Atoms & $X$ & $Y$ & $Z$ \\
\hline C & 2.268885 & -0.526623 & 0.012126 \\
O & 3.115796 & 0.308027 & 0.002204 \\
H & 2.473643 & -1.609225 & 0.035909 \\
C & 0.766762 & -0.209344 & -0.003358 \\
O & -0.016603 & -1.133549 & 0.022714 \\
O & 0.489687 & 1.055294 & -0.043154 \\
H & -0.505520 & 1.222356 & -0.046673 \\
H & -1.849763 & -1.302257 & 0.054742 \\
O & -2.797083 & -1.084863 & 0.077183 \\
H & -3.208855 & -1.588488 & -0.626722 \\
H & -2.514343 & 0.616204 & -0.033310 \\
H & -2.424538 & 2.017445 & 0.644630 \\
O & -2.064862 & 1.487562 & -0.069096 \\
\hline
\end{tabular}

Table S19. Cartesian coordinate of GW.

\begin{tabular}{|c|c|c|c|}
\hline Atoms & $X$ & $Y$ & $Z$ \\
\hline C & 0.753152 & 0.152247 & 0.021681 \\
O & 1.046470 & 1.177502 & -0.520231 \\
O & 1.618142 & -0.797417 & 0.364226 \\
H & 2.497574 & -0.522761 & 0.071931 \\
C & -0.690334 & -0.211143 & 0.373777 \\
H & -0.738952 & -0.658178 & 1.368510 \\
O & -1.475214 & 0.922130 & 0.387706 \\
O & -1.065270 & -1.131827 & -0.612958 \\
H & -1.990135 & -1.354502 & -0.475747 \\
H & -1.138422 & 1.525711 & -0.287391 \\
\hline
\end{tabular}


Table S20. Cartesian coordinate of $(\mathbf{G W})_{1} \mathbf{W}_{1}$.

\begin{tabular}{|c|c|c|c|}
\hline Atoms & $\mathrm{X}$ & $\mathrm{Y}$ & $\mathrm{Z}$ \\
\hline $\mathrm{C}$ & -0.100803 & 0.021841 & -0.164147 \\
$\mathrm{O}$ & -0.553776 & 1.035422 & 0.312599 \\
$\mathrm{O}$ & -0.804089 & -1.006897 & -0.574167 \\
$\mathrm{H}$ & -1.758092 & -0.828473 & -0.399892 \\
$\mathrm{C}$ & 1.408717 & -0.173047 & -0.318411 \\
$\mathrm{H}$ & 1.631543 & -0.653977 & -1.272459 \\
$\mathrm{O}$ & 2.055465 & 1.045564 & -0.302647 \\
$\mathrm{O}$ & 1.762141 & -0.996893 & 0.758689 \\
$\mathrm{H}$ & 2.714931 & -1.120693 & 0.737529 \\
$\mathrm{H}$ & 1.566169 & 1.630128 & 0.290656 \\
$\mathrm{O}$ & -3.169601 & 0.079585 & 0.093383 \\
$\mathrm{H}$ & -3.737118 & -0.159145 & 0.828632 \\
$\mathrm{H}$ & -2.586034 & 0.785154 & 0.408025 \\
\hline
\end{tabular}

Table S21. Cartesian coordinate of $(\mathbf{G W})_{1} \mathbf{W}_{2}$.

\begin{tabular}{|c|c|c|c|}
\hline Atoms & $\mathrm{X}$ & $\mathrm{Y}$ & $\mathrm{Z}$ \\
\hline $\mathrm{C}$ & 0.410404 & -0.136436 & -0.199427 \\
$\mathrm{O}$ & -0.171985 & 0.900405 & 0.038681 \\
$\mathrm{O}$ & -0.136289 & -1.297203 & -0.403205 \\
$\mathrm{H}$ & -1.137525 & -1.267908 & -0.301359 \\
$\mathrm{C}$ & 1.940395 & -0.161849 & -0.260948 \\
$\mathrm{H}$ & 2.274096 & -0.813024 & -1.070377 \\
$\mathrm{O}$ & 2.438453 & 1.101343 & -0.509417 \\
$\mathrm{O}$ & 2.327231 & -0.666352 & 0.988055 \\
$\mathrm{H}$ & 3.287488 & -0.658894 & 1.025762 \\
$\mathrm{H}$ & 1.861566 & 1.734036 & -0.062628 \\
$\mathrm{O}$ & -2.724486 & -1.242450 & -0.154557 \\
\hline
\end{tabular}




\begin{tabular}{|l|l|l|l|}
\hline $\mathrm{H}$ & -3.087545 & -1.768685 & 0.560291 \\
$\mathrm{H}$ & -2.978371 & -0.311887 & 0.023601 \\
$\mathrm{O}$ & -2.881731 & 1.382632 & 0.359638 \\
$\mathrm{H}$ & -3.220931 & 2.053596 & -0.234720 \\
$\mathrm{H}$ & -1.913120 & 1.395476 & 0.268122 \\
\hline
\end{tabular}

Table S22. Cartesian coordinate of $(\mathbf{G A})_{1} \mathbf{A}_{1}$.

\begin{tabular}{|c|c|c|c|}
\hline Atoms & $\mathrm{X}$ & $\mathrm{Y}$ & $\mathrm{Z}$ \\
\hline $\mathrm{C}$ & -1.830481 & 0.299795 & 0.000001 \\
$\mathrm{O}$ & -2.492274 & -0.689611 & -0.000002 \\
$\mathrm{H}$ & -2.253413 & 1.317928 & 0.000015 \\
$\mathrm{C}$ & -0.296509 & 0.300354 & 0.000000 \\
$\mathrm{O}$ & 0.289417 & 1.355794 & -0.000002 \\
$\mathrm{O}$ & 0.240463 & -0.889003 & 0.000001 \\
$\mathrm{H}$ & 1.246976 & -0.789390 & 0.000004 \\
$\mathrm{~N}$ & 2.854420 & -0.291888 & 0.000001 \\
$\mathrm{H}$ & 3.412106 & -0.504680 & 0.817992 \\
$\mathrm{H}$ & 2.662385 & 0.705742 & -0.000017 \\
$\mathrm{H}$ & 3.412097 & -0.504710 & -0.817987 \\
\hline
\end{tabular}

Table S23. Cartesian coordinate of $(\mathbf{G A})_{1} \mathbf{A}_{2}$.

\begin{tabular}{|c|c|c|c|}
\hline Atoms & $\mathrm{X}$ & $\mathrm{Y}$ & $\mathrm{Z}$ \\
\hline $\mathrm{C}$ & -2.2764340 & -0.527586 & 0.002366 \\
$\mathrm{O}$ & -3.1274000 & 0.303790 & -0.012335 \\
$\mathrm{H}$ & -2.4803850 & -1.611003 & 0.015261 \\
$\mathrm{C}$ & -0.7735990 & -0.208338 & 0.004534 \\
$\mathrm{O}$ & 0.0037900 & -1.140096 & 0.012915 \\
$\mathrm{O}$ & -0.4928000 & 1.051646 & -0.003247 \\
$\mathrm{H}$ & 0.5298590 & 1.234946 & -0.002381 \\
\hline
\end{tabular}




\begin{tabular}{|l|l|l|l|}
\hline O & 2.7983530 & -1.214863 & 0.041521 \\
H & 1.8342740 & -1.328790 & 0.031431 \\
H & 3.1679570 & -2.001174 & -0.360567 \\
N & 2.0714330 & 1.592109 & -0.004088 \\
H & 2.6028090 & 0.725826 & -0.099313 \\
H & 2.3622380 & 2.038516 & 0.857677 \\
$H$ & 2.3278720 & 2.208643 & -0.765729 \\
\hline
\end{tabular}

Table S24. Cartesian coordinate of $(\mathbf{G A})_{1}(\mathbf{S A})_{1}$.

\begin{tabular}{|c|c|c|c|}
\hline Atoms & $\mathrm{X}$ & $\mathrm{Y}$ & $\mathrm{Z}$ \\
\hline $\mathrm{C}$ & 3.499741 & 0.424197 & 0.083955 \\
$\mathrm{O}$ & 4.294040 & -0.458070 & 0.116784 \\
$\mathrm{H}$ & 3.754563 & 1.493175 & 0.149405 \\
$\mathrm{C}$ & 1.991415 & 0.177965 & -0.056311 \\
$\mathrm{O}$ & 1.248044 & 1.141253 & -0.078066 \\
$\mathrm{O}$ & 1.653496 & -1.066657 & -0.137542 \\
$\mathrm{H}$ & 0.669790 & -1.162525 & -0.210016 \\
$\mathrm{~S}$ & -1.939394 & -0.105572 & -0.081688 \\
$\mathrm{O}$ & -3.232189 & -0.167175 & -0.643675 \\
$\mathrm{O}$ & -0.995860 & -1.158964 & -0.300894 \\
$\mathrm{O}$ & -2.063102 & -0.018147 & 1.485725 \\
$\mathrm{O}$ & -1.300394 & 1.236074 & -0.468754 \\
$\mathrm{H}$ & -2.870017 & 0.457366 & 1.724169 \\
$\mathrm{H}$ & -0.303245 & 1.221649 & -0.311028 \\
\hline
\end{tabular}

Table S25. Cartesian coordinate of $(\mathbf{G A})_{1}(\mathbf{S A})_{1} \mathbf{W}_{1}$.

\begin{tabular}{|c|c|c|c|}
\hline Atoms & $X$ & $Y$ & $Z$ \\
\hline$C$ & 3.894519 & -0.665606 & -0.001186 \\
O & 4.726787 & -0.021225 & -0.551770 \\
\hline
\end{tabular}




\begin{tabular}{|l|l|l|l|}
\hline H & 4.081624 & -1.635658 & 0.484878 \\
C & 2.430370 & -0.218447 & 0.104854 \\
O & 1.649316 & -0.937850 & 0.698450 \\
O & 2.169503 & 0.910831 & -0.466263 \\
H & 1.209337 & 1.146480 & -0.368161 \\
S & -1.425778 & 0.458594 & 0.184391 \\
O & -2.678302 & 0.910966 & 0.680489 \\
O & -0.403562 & 1.416626 & -0.118629 \\
O & -1.644215 & -0.363683 & -1.107733 \\
O & -0.870004 & -0.560913 & 1.197376 \\
H & -2.557474 & -0.774837 & -1.084633 \\
H & 0.105096 & -0.733525 & 1.024797 \\
O & -4.120178 & -1.071816 & -0.736284 \\
H & -4.217493 & -0.395705 & -0.051767 \\
H & -4.392739 & -1.903436 & -0.342459 \\
\hline
\end{tabular}

Table S26. Cartesian coordinate of $(\mathbf{G A})_{1}(\mathbf{S A})_{1} \mathbf{W}_{2}$.

\begin{tabular}{|c|c|c|c|}
\hline Atoms & $\mathrm{X}$ & $\mathrm{Y}$ & $\mathrm{Z}$ \\
\hline $\mathrm{C}$ & -4.247518 & 0.730018 & 0.244449 \\
$\mathrm{O}$ & -5.117293 & 0.274339 & -0.424124 \\
$\mathrm{H}$ & -4.379090 & 1.569726 & 0.944667 \\
$\mathrm{C}$ & -2.807760 & 0.199608 & 0.215269 \\
$\mathrm{O}$ & -1.978900 & 0.746365 & 0.918505 \\
$\mathrm{O}$ & -2.618705 & -0.801804 & -0.578111 \\
$\mathrm{H}$ & -1.670252 & -1.103978 & -0.555456 \\
$\mathrm{~S}$ & 0.976763 & -0.644549 & -0.023150 \\
$\mathrm{O}$ & 2.238194 & -1.227299 & 0.289858 \\
$\mathrm{O}$ & -0.091380 & -1.521985 & -0.407413 \\
$\mathrm{O}$ & 1.113648 & 0.393395 & -1.138318 \\
\hline
\end{tabular}




\begin{tabular}{|l|l|l|l|}
\hline $\mathrm{O}$ & 0.543488 & 0.169224 & 1.216801 \\
$\mathrm{H}$ & 1.922419 & 1.022652 & -0.981282 \\
$\mathrm{H}$ & -0.425179 & 0.419046 & 1.139136 \\
$\mathrm{O}$ & 3.109962 & 1.855665 & -0.728937 \\
$\mathrm{H}$ & 3.808451 & 1.293991 & -0.314967 \\
$\mathrm{H}$ & 2.966775 & 2.602993 & -0.144029 \\
$\mathrm{O}$ & 4.684796 & 0.092463 & 0.468609 \\
$\mathrm{H}$ & 3.976765 & -0.567582 & 0.530583 \\
$\mathrm{H}$ & 5.433090 & -0.344733 & 0.058480 \\
\hline
\end{tabular}

Table S27. Cartesian coordinate of $(\mathbf{G A})_{1}(\mathbf{S A})_{1} \mathbf{W}_{3}$.

\begin{tabular}{|c|c|c|c|}
\hline Atoms & $\mathrm{X}$ & $\mathrm{Y}$ & $\mathrm{Z}$ \\
\hline $\mathrm{C}$ & 4.074237 & -0.978923 & 0.448564 \\
$\mathrm{O}$ & 4.496255 & -1.886128 & -0.192969 \\
$\mathrm{H}$ & 4.537715 & -0.612732 & 1.378605 \\
$\mathrm{C}$ & 2.819657 & -0.183773 & 0.061965 \\
$\mathrm{O}$ & 2.491184 & 0.749465 & 0.761479 \\
$\mathrm{O}$ & 2.231037 & -0.607042 & -1.011486 \\
$\mathrm{H}$ & 1.401559 & -0.095109 & -1.197017 \\
$\mathrm{~S}$ & -1.162925 & 0.427994 & -0.636186 \\
$\mathrm{O}$ & -2.352381 & -0.044437 & -1.265150 \\
$\mathrm{O}$ & -0.026603 & 0.713624 & -1.468274 \\
$\mathrm{O}$ & -0.661751 & -0.574360 & 0.415129 \\
$\mathrm{O}$ & -1.540741 & 1.680268 & 0.163691 \\
$\mathrm{H}$ & -1.425270 & -0.894970 & 1.034063 \\
$\mathrm{H}$ & -0.688473 & 2.144411 & 0.513466 \\
$\mathrm{O}$ & -2.574910 & -1.349238 & 1.850037 \\
$\mathrm{H}$ & -3.345529 & -1.467726 & 1.244591 \\
$\mathrm{H}$ & -2.849962 & -0.739741 & 2.538567 \\
\hline & & & \\
\hline & & & \\
\hline
\end{tabular}




\begin{tabular}{|l|l|l|l|}
\hline O & -4.428403 & -1.437043 & -0.045558 \\
H & -3.871634 & -0.944369 & -0.669448 \\
H & -4.688437 & -2.245203 & -0.491180 \\
O & 0.612045 & 2.699165 & 0.973566 \\
H & 0.916220 & 3.431313 & 0.431927 \\
H & 1.291388 & 1.998213 & 0.888509 \\
\hline
\end{tabular}

Table S28. Cartesian coordinate of $(\mathbf{G A})_{1}(\mathbf{S A})_{1} \mathbf{A}_{1}$.

\begin{tabular}{|c|c|c|c|}
\hline Atoms & $\mathrm{X}$ & $\mathrm{Y}$ & $\mathrm{Z}$ \\
\hline $\mathrm{C}$ & 3.917446 & -0.642819 & 0.066145 \\
$\mathrm{O}$ & 4.721411 & -0.132275 & -0.644569 \\
$\mathrm{H}$ & 4.138542 & -1.476618 & 0.750983 \\
$\mathrm{C}$ & 2.449210 & -0.200650 & 0.122350 \\
$\mathrm{O}$ & 1.700128 & -0.791243 & 0.876719 \\
$\mathrm{O}$ & 2.151479 & 0.781471 & -0.661821 \\
$\mathrm{H}$ & 1.186546 & 1.023854 & -0.578556 \\
$\mathrm{~S}$ & -1.411187 & 0.482440 & 0.162107 \\
$\mathrm{O}$ & -2.636515 & 1.057748 & 0.596247 \\
$\mathrm{O}$ & -0.382063 & 1.350881 & -0.343252 \\
$\mathrm{O}$ & -1.681086 & -0.576440 & -0.911039 \\
$\mathrm{O}$ & -0.830995 & -0.311797 & 1.356406 \\
$\mathrm{H}$ & -2.682304 & -0.905013 & -0.852285 \\
$\mathrm{H}$ & 0.133338 & -0.523102 & 1.200108 \\
$\mathrm{~N}$ & -4.168810 & -1.177087 & -0.662384 \\
$\mathrm{H}$ & -4.722428 & -1.102089 & -1.507232 \\
$\mathrm{H}$ & -4.426338 & -0.412513 & -0.044044 \\
$\mathrm{H}$ & -4.405497 & -2.049887 & -0.206483 \\
\hline & & & \\
\hline
\end{tabular}

Table S29. Cartesian coordinate of $(\mathbf{G A})_{1}(\mathbf{S A})_{1} \mathbf{A}_{1} \mathbf{W}_{1}$. 


\begin{tabular}{|c|c|c|c|}
\hline Atoms & $x$ & $Y$ & Z \\
\hline C & 4.135356 & 0.646155 & 0.481848 \\
\hline 0 & 4.994569 & -0.170824 & 0.570596 \\
\hline $\mathrm{H}$ & 4.275517 & 1.713478 & 0.717437 \\
\hline C & 2.705553 & 0.322544 & 0.027703 \\
\hline 0 & 1.900118 & 1.233536 & -0.006113 \\
\hline 0 & 2.504492 & -0.914182 & -0.277168 \\
\hline $\mathrm{H}$ & 1.552685 & -1.067842 & -0.574642 \\
\hline$S$ & -0.995149 & -0.477574 & -0.409733 \\
\hline 0 & -2.308913 & -0.643894 & -0.988868 \\
\hline 0 & 0.066489 & -1.213453 & -1.063167 \\
\hline 0 & -1.009513 & -0.707800 & 1.041918 \\
\hline 0 & -0.684820 & 1.051813 & -0.606957 \\
\hline $\mathrm{H}$ & -2.457737 & -0.617653 & 1.489366 \\
\hline $\mathrm{H}$ & 0.272157 & 1.208573 & -0.398003 \\
\hline$N$ & -3.535460 & -0.488605 & 1.553882 \\
\hline $\mathrm{H}$ & -3.958015 & -1.241250 & 1.016140 \\
\hline $\mathrm{H}$ & -3.738561 & 0.409736 & 1.060211 \\
\hline $\mathrm{H}$ & -3.881795 & -0.489878 & 2.506486 \\
\hline 0 & -3.719113 & 1.611883 & -0.202439 \\
\hline $\mathrm{H}$ & -3.270131 & 1.002193 & -0.813199 \\
\hline $\mathrm{H}$ & -3.115441 & 2.355239 & -0.124956 \\
\hline
\end{tabular}

Table S30. Cartesian coordinate of $(\mathbf{G A})_{1}(\mathbf{S A})_{1} \mathbf{A}_{1} \mathbf{W}_{3}$.

\begin{tabular}{|c|c|c|c|}
\hline Atoms & $\mathrm{X}$ & $\mathrm{Y}$ & $\mathrm{Z}$ \\
\hline $\mathrm{C}$ & -3.174897 & -1.452543 & -0.330010 \\
$\mathrm{O}$ & -2.661412 & -2.493345 & -0.622441 \\
H & -4.215298 & -1.206681 & -0.586363 \\
C & -2.392144 & -0.320415 & 0.342952 \\
\hline
\end{tabular}




\begin{tabular}{|c|c|c|c|}
\hline 0 & -2.765351 & 0.815554 & 0.170466 \\
\hline 0 & -1.360941 & -0.735958 & 1.002081 \\
\hline $\mathrm{H}$ & -0.666954 & 0.015446 & 1.184995 \\
\hline$S$ & 1.124563 & 1.094851 & -0.091864 \\
\hline 0 & 2.515633 & 0.718402 & -0.111814 \\
\hline 0 & 0.509575 & 0.880103 & 1.239067 \\
\hline 0 & 0.317304 & 0.442892 & -1.119107 \\
\hline 0 & 1.092516 & 2.617303 & -0.348918 \\
\hline $\mathrm{H}$ & 0.306967 & -1.269494 & -0.941392 \\
\hline $\mathrm{H}$ & 0.141641 & 2.939417 & -0.284202 \\
\hline$N$ & 0.354543 & -2.253281 & -0.590124 \\
\hline $\mathrm{H}$ & 0.781906 & -2.180871 & 0.364850 \\
\hline $\mathrm{H}$ & 0.986049 & -2.787824 & -1.179925 \\
\hline $\mathrm{H}$ & -0.591102 & -2.636943 & -0.540483 \\
\hline 0 & 1.756961 & -1.730067 & 1.710539 \\
\hline $\mathrm{H}$ & 2.603498 & -1.716329 & 1.246093 \\
\hline $\mathrm{H}$ & 1.558404 & -0.801151 & 1.895787 \\
\hline 0 & -1.418214 & 3.246898 & -0.164032 \\
\hline $\mathrm{H}$ & -1.812458 & 3.504043 & -1.000409 \\
\hline $\mathrm{H}$ & -1.873344 & 2.425877 & 0.091071 \\
\hline 0 & 3.108493 & -1.833140 & -0.845148 \\
\hline $\mathrm{H}$ & 3.959359 & -1.953298 & -1.269538 \\
\hline $\mathrm{H}$ & 2.992271 & -0.868234 & -0.742989 \\
\hline
\end{tabular}

Table S31. Cartesian coordinate of $(\mathbf{G A})_{1}(\mathbf{S A})_{1} \mathbf{A}_{1} \mathbf{W}_{4}$.

\begin{tabular}{|c|c|c|c|}
\hline Atoms & $\mathrm{X}$ & $\mathrm{Y}$ & $\mathrm{Z}$ \\
\hline $\mathrm{C}$ & 3.077324 & -1.412009 & 0.108712 \\
$\mathrm{O}$ & 2.573392 & -2.400892 & 0.556618 \\
$\mathrm{H}$ & 4.114392 & -1.115856 & 0.317606 \\
\hline
\end{tabular}




\begin{tabular}{|c|c|c|c|}
\hline$C$ & 2.311367 & -0.434323 & -0.788312 \\
\hline 0 & 2.822613 & 0.588735 & -1.156919 \\
\hline 0 & 1.101321 & -0.854636 & -1.037923 \\
\hline $\mathrm{H}$ & 0.460148 & -0.119235 & -1.313198 \\
\hline$S$ & -1.350024 & 1.125143 & -0.022640 \\
\hline 0 & -2.773635 & 0.939351 & 0.049593 \\
\hline 0 & -0.790756 & 0.782206 & -1.340510 \\
\hline 0 & -0.636929 & 0.391761 & 1.038943 \\
\hline 0 & -1.077293 & 2.625936 & 0.204709 \\
\hline $\mathrm{H}$ & -0.530634 & -1.346885 & 0.926914 \\
\hline $\mathrm{H}$ & -0.082844 & 2.794160 & 0.108682 \\
\hline $\mathrm{N}$ & -0.480825 & -2.335379 & 0.605753 \\
\hline $\mathrm{H}$ & -0.956642 & -2.318270 & -0.329138 \\
\hline $\mathrm{H}$ & -1.005817 & -2.926133 & 1.241818 \\
\hline $\mathrm{H}$ & 0.503130 & -2.603265 & 0.514993 \\
\hline 0 & -2.011985 & -1.863067 & -1.610283 \\
\hline $\mathrm{H}$ & -2.806121 & -1.780672 & -1.064619 \\
\hline $\mathrm{H}$ & -1.788526 & -0.955633 & -1.861581 \\
\hline 0 & 1.487529 & 2.816228 & 0.005138 \\
\hline $\mathrm{H}$ & 1.869965 & 2.246363 & 0.692055 \\
\hline $\mathrm{H}$ & 1.823141 & 2.433076 & -0.814903 \\
\hline 0 & -3.250306 & -1.634222 & 0.939126 \\
\hline $\mathrm{H}$ & -4.095686 & -1.782543 & 1.366106 \\
\hline $\mathrm{H}$ & -3.186785 & -0.670224 & 0.802621 \\
\hline 0 & 1.958843 & 0.645975 & 1.761237 \\
\hline $\mathrm{H}$ & 2.100232 & 0.698416 & 2.708257 \\
\hline $\mathrm{H}$ & 0.993709 & 0.571056 & 1.626117 \\
\hline
\end{tabular}

Table S32. Cartesian coordinate of $(\mathbf{G A})_{1} \mathbf{A}_{2}$. 


\begin{tabular}{|c|c|c|c|}
\hline Atoms & $\mathrm{X}$ & $\mathrm{Y}$ & $\mathrm{Z}$ \\
\hline $\mathrm{H}$ & -0.412898 & 1.279890 & 0.005150 \\
$\mathrm{~N}$ & -1.954746 & 1.668330 & -0.029875 \\
$\mathrm{H}$ & -2.211614 & 2.156758 & -0.879561 \\
$\mathrm{H}$ & -2.232134 & 2.255488 & 0.747796 \\
$\mathrm{H}$ & -2.501960 & 0.801717 & 0.013954 \\
$\mathrm{~N}$ & -3.064692 & -1.161282 & 0.067091 \\
$\mathrm{H}$ & -3.542597 & -1.602327 & -0.708898 \\
$\mathrm{H}$ & -3.423940 & -1.585940 & 0.913065 \\
$\mathrm{H}$ & -2.079154 & -1.404296 & -0.001634 \\
$\mathrm{C}$ & 2.304774 & -0.603274 & -0.005945 \\
$\mathrm{O}$ & 3.196208 & 0.182480 & 0.064298 \\
$\mathrm{H}$ & 2.457511 & -1.694139 & -0.060791 \\
$\mathrm{C}$ & 0.818889 & -0.213921 & -0.030466 \\
$\mathrm{O}$ & -0.003355 & -1.099835 & -0.101845 \\
$\mathrm{O}$ & 0.599755 & 1.060690 & 0.028656 \\
\hline & & & \\
\hline
\end{tabular}

Table S33. Cartesian coordinate of $(\mathbf{G A})_{1} \mathbf{A}_{2} \mathbf{W}_{1}$.

\begin{tabular}{|c|c|c|c|}
\hline Atoms & $\mathrm{X}$ & $\mathrm{Y}$ & $\mathrm{Z}$ \\
\hline $\mathrm{H}$ & -0.106502 & 1.405760 & -0.126554 \\
$\mathrm{~N}$ & -1.530015 & 1.752068 & -0.111556 \\
$\mathrm{H}$ & -1.988269 & 1.403620 & -0.948962 \\
$\mathrm{H}$ & -1.734410 & 2.737784 & -0.005629 \\
$\mathrm{H}$ & -1.941556 & 1.230712 & 0.669362 \\
$\mathrm{~N}$ & -2.377110 & -0.553341 & 1.610520 \\
$\mathrm{H}$ & -2.814324 & -0.845714 & 2.474919 \\
$\mathrm{H}$ & -1.475322 & -1.014578 & 1.547324 \\
$\mathrm{H}$ & -2.927347 & -0.915887 & 0.838455 \\
$\mathrm{C}$ & 2.563193 & -0.611511 & 0.071342 \\
\hline
\end{tabular}




\begin{tabular}{|l|l|l|l|}
\hline O & 3.487468 & 0.128678 & 0.188648 \\
H & 2.660526 & -1.710080 & 0.059187 \\
$\mathrm{C}$ & 1.107135 & -0.139781 & -0.078810 \\
$\mathrm{O}$ & 0.247446 & -1.002475 & -0.177280 \\
$\mathrm{O}$ & 0.941133 & 1.129470 & -0.084012 \\
$\mathrm{O}$ & -2.257148 & -0.747698 & -1.401010 \\
$\mathrm{H}$ & -1.375038 & -0.894856 & -1.018497 \\
$\mathrm{H}$ & -2.321040 & -1.343902 & -2.148300 \\
\hline
\end{tabular}

Table S34. Cartesian coordinate of $(\mathbf{G A})_{1}(\mathbf{S A})_{2}$.

\begin{tabular}{|c|c|c|c|}
\hline Atoms & $x$ & $Y$ & Z \\
\hline C & -4.854272 & -1.352555 & 0.038540 \\
\hline 0 & -5.901396 & -0.854907 & 0.292700 \\
\hline $\mathrm{H}$ & -4.697300 & -2.429376 & -0.125371 \\
\hline C & -3.564360 & -0.530539 & -0.094993 \\
\hline 0 & -2.529495 & -1.118001 & -0.359996 \\
\hline $\mathrm{O}$ & -3.712169 & 0.737862 & 0.093465 \\
\hline $\mathrm{H}$ & -2.855691 & 1.221955 & -0.002750 \\
\hline$S$ & -0.069693 & 1.178428 & -0.076187 \\
\hline 0 & 1.121071 & 1.758953 & -0.598911 \\
\hline 0 & -1.303871 & 1.879646 & -0.217977 \\
\hline 0 & 0.090685 & 0.867153 & 1.421753 \\
\hline 0 & -0.193328 & -0.217934 & -0.740876 \\
\hline $\mathrm{H}$ & 0.943911 & 0.366823 & 1.548723 \\
\hline $\mathrm{H}$ & -1.129274 & -0.607249 & -0.591836 \\
\hline$S$ & 3.238374 & -0.673195 & 0.139466 \\
\hline $\mathrm{O}$ & 2.307834 & -0.486391 & 1.221733 \\
\hline 0 & 4.406303 & -1.425395 & 0.338897 \\
\hline 0 & 3.658443 & 0.719548 & -0.397570 \\
\hline
\end{tabular}




\begin{tabular}{|l|l|l|l|}
\hline $\mathrm{O}$ & 2.451941 & -1.308290 & -1.048875 \\
$\mathrm{H}$ & 2.870020 & 1.291448 & -0.504056 \\
$\mathrm{H}$ & 1.513075 & -1.046722 & -1.033200 \\
\hline
\end{tabular}

Table S35. Cartesian coordinate of $(\mathbf{G A})_{1}(\mathbf{S A})_{2} \mathbf{W}_{1}$.

\begin{tabular}{|c|c|c|c|}
\hline Atoms & $x$ & $Y$ & Z \\
\hline C & 5.444169 & 0.417658 & 0.955211 \\
\hline 0 & 6.371745 & -0.289636 & 0.735193 \\
\hline $\mathrm{H}$ & 5.472713 & 1.297238 & 1.615973 \\
\hline C & 4.062144 & 0.184202 & 0.328559 \\
\hline 0 & 3.162411 & 0.956746 & 0.607401 \\
\hline 0 & 3.991814 & -0.840340 & -0.454932 \\
\hline $\mathrm{H}$ & 3.081846 & -0.948470 & -0.825062 \\
\hline$S$ & 0.341740 & -0.332651 & -0.760639 \\
\hline 0 & -0.816983 & -0.095546 & -1.577159 \\
\hline 0 & 1.471961 & -0.961541 & -1.365798 \\
\hline $\mathrm{O}$ & -0.041635 & -1.161527 & 0.461682 \\
\hline 0 & 0.749881 & 1.049152 & -0.212343 \\
\hline $\mathrm{H}$ & -0.920363 & -0.821578 & 0.863924 \\
\hline $\mathrm{H}$ & 1.708908 & 1.030132 & 0.129822 \\
\hline$S$ & -3.489600 & -0.289920 & 0.593502 \\
\hline 0 & -2.199581 & -0.140128 & 1.245163 \\
\hline 0 & -4.534906 & -0.930799 & 1.279219 \\
\hline 0 & -3.247219 & -1.068164 & -0.733381 \\
\hline 0 & -3.946197 & 1.104821 & 0.157250 \\
\hline $\mathrm{H}$ & -2.414170 & -0.785394 & -1.167194 \\
\hline $\mathrm{H}$ & -3.165319 & 1.677330 & -0.155394 \\
\hline 0 & -1.922590 & 2.434254 & -0.669810 \\
\hline $\mathrm{H}$ & -1.465447 & 1.864792 & -1.302839 \\
\hline
\end{tabular}




\begin{tabular}{|l|l|l|l|}
\hline $\mathrm{H}$ & -1.279897 & 2.577589 & 0.032464 \\
\hline
\end{tabular}

Table S36. Cartesian coordinate of $(\mathbf{G A})_{1}(\mathbf{S A})_{2} \mathbf{W}_{2}$.

\begin{tabular}{|c|c|c|c|}
\hline Atoms & $x$ & Y & Z \\
\hline C & 5.4932720 & -0.050475 & -1.190379 \\
\hline 0 & 6.5186910 & 0.041457 & -0.598877 \\
\hline $\mathrm{H}$ & 5.4068420 & -0.074961 & -2.287364 \\
\hline C & 4.1350740 & -0.146990 & -0.481477 \\
\hline 0 & 3.1311360 & -0.228306 & -1.166352 \\
\hline $\mathrm{O}$ & 4.1979650 & -0.129128 & 0.808418 \\
\hline $\mathrm{H}$ & 3.2960640 & -0.187496 & 1.215455 \\
\hline$S$ & 0.5563540 & 0.069944 & 0.995156 \\
\hline 0 & -0.7113110 & -0.407502 & 1.470199 \\
\hline 0 & 1.7109010 & -0.317059 & 1.748215 \\
\hline 0 & 0.5708120 & 1.575303 & 0.859158 \\
\hline 0 & 0.6825660 & -0.470960 & -0.450792 \\
\hline $\mathrm{H}$ & -0.1655480 & 1.968778 & 0.187821 \\
\hline $\mathrm{H}$ & 1.6405420 & -0.381596 & -0.772218 \\
\hline$S$ & -3.6379730 & 0.036246 & -0.390801 \\
\hline 0 & -2.4725080 & 0.242964 & -1.225249 \\
\hline 0 & -4.8431160 & 0.694144 & -0.703006 \\
\hline $\mathrm{O}$ & -3.2441930 & 0.468915 & 1.055266 \\
\hline 0 & -3.8810460 & -1.474049 & -0.300433 \\
\hline $\mathrm{H}$ & -2.3329540 & 0.170877 & 1.275300 \\
\hline $\mathrm{H}$ & -2.9939220 & -1.973143 & -0.346567 \\
\hline 0 & -1.0833350 & 2.440020 & -0.729100 \\
\hline $\mathrm{H}$ & -1.6400240 & 1.686959 & -1.039798 \\
\hline $\mathrm{H}$ & -1.6814670 & 3.119760 & -0.406455 \\
\hline 0 & -1.5472430 & -2.505861 & -0.397696 \\
\hline
\end{tabular}




\begin{tabular}{|l|l|l|l|}
\hline $\mathrm{H}$ & -1.1645850 & -2.440865 & 0.484752 \\
$\mathrm{H}$ & -1.0636860 & -1.842066 & -0.907483 \\
\hline
\end{tabular}

Table S37. Cartesian coordinate of $(\mathbf{G A})_{1}(\mathbf{S A})_{2} \mathbf{W}_{3}$.

\begin{tabular}{|c|c|c|c|}
\hline Atoms & $x$ & $Y$ & Z \\
\hline C & 5.576830 & 0.446340 & -1.141497 \\
\hline 0 & 6.581122 & 0.606250 & -0.527597 \\
\hline $\mathrm{H}$ & 5.497121 & 0.543382 & -2.235418 \\
\hline C & 4.242302 & 0.078537 & -0.478375 \\
\hline 0 & 3.268135 & -0.067869 & -1.194012 \\
\hline 0 & 4.291315 & -0.039584 & 0.805767 \\
\hline $\mathrm{H}$ & 3.398072 & -0.279802 & 1.182011 \\
\hline$S$ & 0.695173 & -0.303453 & 1.000734 \\
\hline $\mathrm{O}$ & -0.482292 & -1.054752 & 1.427416 \\
\hline $\mathrm{O}$ & 1.905644 & -0.706680 & 1.668229 \\
\hline 0 & 0.472723 & 1.140194 & 1.060010 \\
\hline $\mathrm{O}$ & 0.834327 & -0.679420 & -0.512955 \\
\hline $\mathrm{H}$ & -0.363994 & 1.821520 & 0.108053 \\
\hline $\mathrm{H}$ & 1.762917 & -0.459548 & -0.824331 \\
\hline$S$ & -3.223783 & -0.261356 & -0.425818 \\
\hline 0 & -2.109307 & -0.020780 & -1.322229 \\
\hline 0 & -4.400771 & 0.514763 & -0.613504 \\
\hline 0 & -2.748911 & 0.013282 & 1.015816 \\
\hline 0 & -3.561605 & -1.744116 & -0.488304 \\
\hline $\mathrm{H}$ & -1.842367 & -0.406897 & 1.220953 \\
\hline $\mathrm{H}$ & -2.681742 & -2.289393 & -0.567262 \\
\hline 0 & -0.999477 & 2.204969 & -0.627007 \\
\hline $\mathrm{H}$ & -1.341151 & 1.387333 & -1.092482 \\
\hline $\mathrm{H}$ & -1.834647 & 2.612177 & -0.209493 \\
\hline
\end{tabular}




\begin{tabular}{|l|l|l|l|}
\hline O & -1.300715 & -2.846904 & -0.617065 \\
H & -0.894374 & -2.659400 & 0.242520 \\
H & -0.800170 & -2.284312 & -1.222592 \\
O & -3.262660 & 3.000523 & 0.268399 \\
$H$ & -3.368611 & 3.016086 & 1.223701 \\
$H$ & -3.888314 & 2.327519 & -0.048809 \\
\hline
\end{tabular}

Table S38. Cartesian coordinate of $(\mathbf{G A})_{1}(\mathbf{S A})_{2} \mathbf{W}_{4}$.

\begin{tabular}{|c|c|c|c|}
\hline Atoms & $x$ & $Y$ & Z \\
\hline C & -5.816144 & 0.482879 & 0.932317 \\
\hline 0 & -6.749814 & 0.783691 & 0.262263 \\
\hline $\mathrm{H}$ & -5.830991 & 0.440126 & 2.032500 \\
\hline C & -4.450336 & 0.110708 & 0.338677 \\
\hline 0 & -3.552204 & -0.183536 & 1.107962 \\
\hline 0 & -4.392187 & 0.150030 & -0.948730 \\
\hline $\mathrm{H}$ & -3.483117 & -0.103247 & -1.280489 \\
\hline$S$ & -0.830936 & -0.247141 & -0.886667 \\
\hline 0 & 0.381202 & -0.941147 & -1.308091 \\
\hline 0 & -1.988122 & -0.568685 & -1.683361 \\
\hline 0 & -0.616072 & 1.184459 & -0.744440 \\
\hline 0 & -1.103005 & -0.810943 & 0.558660 \\
\hline $\mathrm{H}$ & 0.316886 & 1.834560 & 0.271418 \\
\hline $\mathrm{H}$ & -2.050902 & -0.587371 & 0.815492 \\
\hline$S$ & 3.323326 & 0.005651 & 0.116650 \\
\hline 0 & 2.304829 & -0.030071 & 1.188413 \\
\hline 0 & 4.376512 & 0.943720 & 0.355077 \\
\hline 0 & 2.572967 & 0.581077 & -1.138401 \\
\hline 0 & 3.757397 & -1.346672 & -0.207635 \\
\hline $\mathrm{H}$ & 1.754982 & 0.057050 & -1.321672 \\
\hline
\end{tabular}




\begin{tabular}{|l|l|l|l|}
\hline $\mathrm{H}$ & 2.710887 & -2.368629 & -0.338401 \\
$\mathrm{O}$ & 0.988003 & 2.131120 & 0.978203 \\
$\mathrm{H}$ & 1.479251 & 1.272360 & 1.218947 \\
$\mathrm{H}$ & 1.721499 & 2.721188 & 0.563099 \\
$\mathrm{O}$ & 1.858119 & -2.964650 & -0.348498 \\
$\mathrm{H}$ & 1.228408 & -2.484318 & -0.929521 \\
$\mathrm{H}$ & 1.450241 & -2.825698 & 0.616109 \\
$\mathrm{O}$ & 3.005906 & 3.367664 & 0.076636 \\
$\mathrm{H}$ & 2.996905 & 3.585635 & -0.859432 \\
$\mathrm{H}$ & 3.683713 & 2.671502 & 0.174101 \\
$\mathrm{O}$ & 0.930056 & -2.343997 & 1.865405 \\
$\mathrm{H}$ & 0.063699 & -1.970677 & 1.644226 \\
$\mathrm{H}$ & 1.490474 & -1.556643 & 1.980245 \\
\hline
\end{tabular}

Table S39. Cartesian coordinate of $(\mathbf{G A})_{1}(\mathbf{S A})_{2} \mathbf{W}_{5}$.

\begin{tabular}{|c|c|c|c|}
\hline Atoms & $X$ & $Y$ & $Z$ \\
\hline C & 6.056714 & -0.589161 & 0.783407 \\
O & 6.963551 & -0.714271 & 0.026177 \\
H & 6.134627 & -0.733084 & 1.872268 \\
C & 4.641794 & -0.202745 & 0.331409 \\
O & 3.778269 & -0.088487 & 1.182760 \\
O & 4.510032 & -0.033694 & -0.940629 \\
H & 3.572870 & 0.222113 & -1.178357 \\
S & 0.939022 & 0.188752 & -0.637897 \\
O & -0.300135 & 0.928060 & -0.865588 \\
$O$ & 2.043029 & 0.671017 & -1.433998 \\
$O$ & 0.751271 & -1.248540 & -0.733124 \\
$O$ & 1.276346 & 0.511800 & 0.861310 \\
H & -0.234999 & -2.091432 & 0.094750 \\
\hline
\end{tabular}




\begin{tabular}{|c|c|c|c|}
\hline $\mathrm{H}$ & 2.239937 & 0.286736 & 1.039865 \\
\hline$S$ & -3.328812 & -0.365751 & 0.162010 \\
\hline 0 & -2.361598 & -0.531567 & 1.271085 \\
\hline 0 & -4.298536 & -1.419029 & 0.095124 \\
\hline 0 & -2.477643 & -0.546922 & -1.146102 \\
\hline 0 & -3.880212 & 0.976543 & 0.157585 \\
\hline $\mathrm{H}$ & -1.666349 & 0.021231 & -1.115018 \\
\hline $\mathrm{H}$ & -3.060502 & 2.234497 & 0.400761 \\
\hline 0 & -0.940422 & -2.540458 & 0.669144 \\
\hline $\mathrm{H}$ & -1.489614 & -1.767172 & 1.051042 \\
\hline $\mathrm{H}$ & -1.609933 & -3.054501 & 0.078148 \\
\hline 0 & -2.417313 & 2.987860 & 0.637304 \\
\hline $\mathrm{H}$ & -1.915133 & 3.268606 & -0.191521 \\
\hline $\mathrm{H}$ & -1.746558 & 2.544224 & 1.307161 \\
\hline 0 & -2.801654 & -3.617676 & -0.657779 \\
\hline $\mathrm{H}$ & -2.708321 & -3.601961 & -1.614004 \\
\hline $\mathrm{H}$ & -3.515716 & -2.981230 & -0.453062 \\
\hline 0 & -0.948446 & 3.523480 & -1.394780 \\
\hline $\mathrm{H}$ & -1.244298 & 3.798628 & -2.264601 \\
\hline $\mathrm{H}$ & -0.513574 & 2.659592 & -1.489018 \\
\hline 0 & -0.976861 & 1.695049 & 2.199826 \\
\hline $\mathrm{H}$ & -1.459025 & 0.853365 & 2.108338 \\
\hline $\mathrm{H}$ & -0.095239 & 1.498487 & 1.852031 \\
\hline
\end{tabular}

Table S40. Cartesian coordinate of $(\mathbf{G A})_{1}(\mathbf{S A})_{2} \mathbf{W}_{6}$.

\begin{tabular}{|c|c|c|c|}
\hline Atoms & $X$ & $Y$ & $Z$ \\
\hline C & -6.054094 & -0.356575 & -0.881206 \\
O & -6.980782 & -0.471460 & -0.146843 \\
H & -6.106584 & -0.496658 & -1.972055 \\
\hline
\end{tabular}




\begin{tabular}{|c|c|c|c|}
\hline$C$ & -4.645706 & 0.008605 & -0.392496 \\
\hline $\mathrm{O}$ & -3.758448 & 0.110681 & -1.220660 \\
\hline $\mathrm{O}$ & -4.543337 & 0.174063 & 0.882896 \\
\hline $\mathrm{H}$ & -3.608207 & 0.413749 & 1.142803 \\
\hline$S$ & -0.946651 & 0.360502 & 0.663677 \\
\hline $\mathrm{O}$ & 0.272706 & 1.129296 & 0.917475 \\
\hline $\mathrm{O}$ & -2.074882 & 0.833046 & 1.432013 \\
\hline $\mathrm{O}$ & -0.717936 & -1.069206 & 0.769224 \\
\hline 0 & -1.252575 & 0.663059 & -0.844334 \\
\hline $\mathrm{H}$ & -0.232051 & -2.079011 & -0.767871 \\
\hline $\mathrm{H}$ & -2.218871 & 0.461376 & -1.036188 \\
\hline$S$ & 3.164967 & -0.337669 & -0.428155 \\
\hline 0 & 2.103820 & -0.300785 & -1.429009 \\
\hline $\mathrm{O}$ & 3.895619 & -1.586386 & -0.400986 \\
\hline 0 & 2.436510 & -0.297294 & 0.968739 \\
\hline $\mathrm{O}$ & 4.023798 & 0.832329 & -0.490697 \\
\hline $\mathrm{H}$ & 1.610534 & 0.262174 & 0.920785 \\
\hline $\mathrm{H}$ & 3.319631 & 2.180185 & -0.467003 \\
\hline 0 & 0.374607 & -2.483432 & -1.410962 \\
\hline $\mathrm{H}$ & 0.875814 & -1.721279 & -1.746609 \\
\hline $\mathrm{H}$ & 1.411548 & -3.144742 & -0.613117 \\
\hline $\mathrm{O}$ & 2.714656 & 3.003109 & -0.515159 \\
\hline $\mathrm{H}$ & 2.332133 & 3.210026 & 0.393737 \\
\hline $\mathrm{H}$ & 1.929974 & 2.732903 & -1.153606 \\
\hline $\mathrm{O}$ & 2.170855 & -3.444430 & 0.040680 \\
\hline $\mathrm{H}$ & 1.811998 & -3.199003 & 0.966329 \\
\hline $\mathrm{H}$ & 2.943611 & -2.815268 & -0.142192 \\
\hline O & 1.484425 & 3.441738 & 1.696741 \\
\hline $\mathrm{H}$ & 1.867491 & 3.501908 & 2.573947 \\
\hline
\end{tabular}




\begin{tabular}{|c|c|c|c|}
\hline$H$ & 0.854148 & 2.703068 & 1.700826 \\
$\mathrm{O}$ & 0.963536 & 2.118448 & -2.048780 \\
$\mathrm{H}$ & 1.322121 & 1.211357 & -2.073223 \\
$\mathrm{H}$ & 0.085246 & 2.002558 & -1.662514 \\
$\mathrm{O}$ & 1.174795 & -2.590662 & 2.229481 \\
$\mathrm{H}$ & 1.805729 & -1.901697 & 2.467056 \\
$\mathrm{H}$ & 0.402550 & -2.096083 & 1.904205 \\
\hline
\end{tabular}

Table S41. Cartesian coordinate of $(\mathbf{G A})_{1}(\mathbf{S A})_{2} \mathbf{W}_{7}$.

\begin{tabular}{|c|c|c|c|}
\hline Atoms & $x$ & $Y$ & Z \\
\hline C & -6.166851 & -0.191895 & -0.934446 \\
\hline 0 & -7.119785 & -0.149262 & -0.226139 \\
\hline $\mathrm{H}$ & -6.204463 & -0.422044 & -2.010546 \\
\hline C & -4.741670 & 0.074498 & -0.430691 \\
\hline 0 & -3.829041 & 0.017176 & -1.235415 \\
\hline 0 & -4.653651 & 0.338235 & 0.828822 \\
\hline $\mathrm{H}$ & -3.703793 & 0.502970 & 1.098567 \\
\hline$S$ & -1.037760 & 0.204021 & 0.701466 \\
\hline 0 & 0.211461 & 0.924792 & 0.943221 \\
\hline 0 & -2.153464 & 0.795358 & 1.407703 \\
\hline 0 & -0.902406 & -1.225541 & 0.897091 \\
\hline 0 & -1.300321 & 0.425895 & -0.832300 \\
\hline $\mathrm{H}$ & -0.597277 & -2.300550 & -0.697170 \\
\hline $\mathrm{H}$ & -2.273708 & 0.272061 & -1.031449 \\
\hline $\mathrm{S}$ & 3.037266 & -0.764988 & -0.426424 \\
\hline 0 & 1.898627 & -0.676872 & -1.337842 \\
\hline 0 & 3.560608 & -2.113447 & -0.299890 \\
\hline 0 & 2.452335 & -0.438930 & 1.006022 \\
\hline 0 & 4.055463 & 0.216793 & -0.702907 \\
\hline
\end{tabular}




\begin{tabular}{|c|c|c|c|}
\hline $\mathrm{H}$ & 1.620502 & 0.101358 & 0.936497 \\
\hline $\mathrm{H}$ & 4.255593 & 1.753026 & -0.088810 \\
\hline 0 & -0.010661 & -2.719507 & -1.346358 \\
\hline $\mathrm{H}$ & 0.548632 & -1.980893 & -1.641988 \\
\hline $\mathrm{H}$ & 0.983600 & -3.479622 & -0.538742 \\
\hline 0 & 1.972881 & 3.529132 & -0.563869 \\
\hline $\mathrm{H}$ & 1.386739 & 3.642881 & 0.247445 \\
\hline $\mathrm{H}$ & 1.541162 & 2.796816 & -1.187519 \\
\hline 0 & 1.730373 & -3.819960 & 0.096807 \\
\hline $\mathrm{H}$ & 1.448801 & -3.493583 & 1.025277 \\
\hline $\mathrm{H}$ & 2.540009 & -3.234893 & -0.117262 \\
\hline 0 & 0.326617 & 3.616153 & 1.401069 \\
\hline $\mathrm{H}$ & 0.477973 & 3.957018 & 2.284360 \\
\hline $\mathrm{H}$ & 0.120214 & 2.668818 & 1.482023 \\
\hline 0 & 0.950446 & 1.787025 & -2.003410 \\
\hline $\mathrm{H}$ & 1.404733 & 0.927123 & -1.889522 \\
\hline $\mathrm{H}$ & 0.044151 & 1.590967 & -1.727963 \\
\hline 0 & 1.065171 & -2.681947 & 2.272520 \\
\hline $\mathrm{H}$ & 1.758538 & -2.009880 & 2.304842 \\
\hline $\mathrm{H}$ & 0.273600 & -2.184193 & 2.004860 \\
\hline 0 & 4.231930 & 2.703487 & 0.169872 \\
\hline $\mathrm{H}$ & 2.914894 & 3.236258 & -0.277881 \\
\hline $\mathrm{H}$ & 5.026478 & 3.107572 & -0.184840 \\
\hline
\end{tabular}

Table S42. Cartesian coordinate of $(\mathbf{G W})_{1} \mathbf{A}_{1}$.

\begin{tabular}{|c|c|c|c|}
\hline Atoms & $X$ & $Y$ & $Z$ \\
\hline$C$ & 0.065134 & 0.077338 & 0.1451150 \\
$O$ & 0.449325 & 1.105055 & -0.3612940 \\
$O$ & 0.827708 & -0.900381 & 0.5618290 \\
\hline
\end{tabular}




\begin{tabular}{|l|r|r|l|}
\hline $\mathrm{H}$ & 1.789455 & -0.673973 & 0.3582680 \\
$\mathrm{C}$ & -1.430931 & -0.188863 & 0.3346450 \\
$\mathrm{H}$ & -1.615803 & -0.638909 & 1.3118620 \\
$\mathrm{O}$ & -2.138632 & 0.995398 & 0.2732230 \\
$\mathrm{O}$ & -1.766729 & -1.077804 & -0.6974430 \\
$\mathrm{H}$ & -2.717843 & -1.211872 & -0.6703750 \\
$\mathrm{H}$ & -1.650346 & 1.589747 & -0.3122220 \\
$\mathrm{~N}$ & 3.264842 & -0.001907 & -0.1359680 \\
$\mathrm{H}$ & 2.862377 & 0.865724 & -0.4789930 \\
$\mathrm{H}$ & 3.738872 & -0.453503 & -0.9084890 \\
$\mathrm{H}$ & 3.960802 & 0.227143 & 0.5626360 \\
\hline
\end{tabular}

Table S43. Cartesian coordinate of $(\mathbf{G W})_{1} \mathbf{A}_{1} \mathbf{W}_{1}$.

\begin{tabular}{|c|c|c|c|}
\hline Atoms & $\mathrm{X}$ & $\mathrm{Y}$ & $\mathrm{Z}$ \\
\hline $\mathrm{C}$ & 0.416772 & -0.162910 & -0.202417 \\
$\mathrm{O}$ & -0.155125 & 0.898308 & -0.054405 \\
$\mathrm{O}$ & -0.137428 & -1.327361 & -0.318916 \\
$\mathrm{H}$ & -1.167377 & -1.290429 & -0.230664 \\
$\mathrm{C}$ & 1.948327 & -0.198496 & -0.242124 \\
$\mathrm{H}$ & 2.291987 & -0.914410 & -0.990444 \\
$\mathrm{O}$ & 2.454541 & 1.040587 & -0.582640 \\
$\mathrm{O}$ & 2.321524 & -0.601277 & 1.048833 \\
$\mathrm{H}$ & 3.280593 & -0.568944 & 1.100010 \\
$\mathrm{H}$ & 1.848316 & 1.704667 & -0.228775 \\
$\mathrm{~N}$ & -2.758548 & -1.318474 & -0.082650 \\
$\mathrm{H}$ & -3.071114 & -0.378420 & 0.163482 \\
$\mathrm{H}$ & -3.066771 & -1.955819 & 0.641767 \\
$\mathrm{H}$ & -3.227380 & -1.584698 & -0.940251 \\
$\mathrm{O}$ & -2.847421 & 1.567420 & 0.249522 \\
\hline
\end{tabular}




\begin{tabular}{|l|l|l|l|}
\hline $\mathrm{H}$ & -2.970101 & 2.310856 & 0.840191 \\
$\mathrm{H}$ & -1.887639 & 1.453540 & 0.151332 \\
\hline
\end{tabular}

Table S44. Cartesian coordinate of $(\mathbf{G W})_{1}(\mathbf{S A})_{1}$.

\begin{tabular}{|c|c|c|c|}
\hline Atoms & $x$ & $Y$ & Z \\
\hline C & 1.903852 & 0.701398 & -0.184520 \\
\hline 0 & 0.999596 & 1.274481 & -0.729725 \\
\hline $\mathrm{O}$ & 2.894162 & 1.311830 & 0.444767 \\
\hline $\mathrm{H}$ & 2.738286 & 2.265983 & 0.421979 \\
\hline C & 2.062464 & -0.821089 & -0.098219 \\
\hline $\mathrm{H}$ & 3.105514 & -1.072577 & -0.292851 \\
\hline 0 & 1.759815 & -1.287408 & 1.158790 \\
\hline 0 & 1.227675 & -1.359361 & -1.096843 \\
\hline $\mathrm{H}$ & 1.281168 & -2.318955 & -1.032538 \\
\hline $\mathrm{H}$ & 0.846992 & -1.026135 & 1.372128 \\
\hline$S$ & -1.807924 & 0.015139 & 0.171762 \\
\hline 0 & -0.807682 & -0.251725 & 1.165125 \\
\hline $\mathrm{O}$ & -3.165406 & -0.240044 & 0.439441 \\
\hline 0 & -1.410124 & -0.740690 & -1.125927 \\
\hline 0 & -1.702439 & 1.515663 & -0.241775 \\
\hline $\mathrm{H}$ & -0.432605 & -0.873868 & -1.167808 \\
\hline $\mathrm{H}$ & -0.775231 & 1.719507 & -0.463486 \\
\hline
\end{tabular}

Table S45. Cartesian coordinate of $(\mathbf{G W})_{1}(\mathbf{S A})_{1} \mathbf{W}_{1}$.

\begin{tabular}{|c|c|c|c|}
\hline Atoms & $\mathrm{X}$ & $\mathrm{Y}$ & $\mathrm{Z}$ \\
\hline $\mathrm{C}$ & 1.492559 & -0.566742 & 0.463748 \\
$\mathrm{O}$ & 0.877115 & -1.181547 & -0.381900 \\
$\mathrm{O}$ & 1.006381 & -0.062878 & 1.556938 \\
$\mathrm{H}$ & 0.003937 & -0.077698 & 1.528915 \\
\hline
\end{tabular}




\begin{tabular}{|l|l|l|l|}
\hline C & 2.969177 & -0.240681 & 0.237126 \\
H & 3.534408 & -0.300311 & 1.166620 \\
O & 3.538147 & -1.091361 & -0.673862 \\
O & 2.923558 & 1.098639 & -0.225961 \\
H & 3.799067 & 1.337993 & -0.544608 \\
H & 2.860217 & -1.351249 & -1.311981 \\
S & -2.251789 & -0.115600 & -0.012143 \\
O & -1.537771 & 0.210520 & 1.205554 \\
O & -3.658284 & -0.125356 & 0.007974 \\
O & -1.780503 & -1.513584 & -0.488227 \\
O & -1.748614 & 0.836353 & -1.114850 \\
H & -0.793676 & -1.541690 & -0.486429 \\
H & -0.916336 & 1.336972 & -0.843718 \\
H & 1.226198 & 1.938783 & -0.452969 \\
H & 0.190292 & 2.389522 & 0.593139 \\
O & 0.299233 & 2.199441 & -0.343157 \\
\hline
\end{tabular}

Table S46. Cartesian coordinate of $(\mathbf{G W})_{1}(\mathbf{S A})_{1} \mathbf{W}_{2}$.

\begin{tabular}{|c|c|c|c|}
\hline Atoms & $\mathrm{X}$ & $\mathrm{Y}$ & $\mathrm{Z}$ \\
\hline $\mathrm{C}$ & -2.363907 & -0.201274 & 0.186703 \\
$\mathrm{O}$ & -1.555112 & 0.600101 & 0.619529 \\
$\mathrm{O}$ & -2.106808 & -1.407472 & -0.209880 \\
$\mathrm{H}$ & -1.138097 & -1.617685 & -0.138951 \\
$\mathrm{C}$ & -3.835457 & 0.195812 & 0.054052 \\
$\mathrm{H}$ & -4.471443 & -0.660233 & 0.282820 \\
$\mathrm{O}$ & -4.157171 & 1.196787 & 0.946908 \\
$\mathrm{O}$ & -3.958232 & 0.587549 & -1.282999 \\
$\mathrm{H}$ & -4.869135 & 0.853043 & -1.436738 \\
$\mathrm{H}$ & -3.425496 & 1.825962 & 0.968047 \\
\hline
\end{tabular}




\begin{tabular}{|l|l|l|l|}
\hline $\mathrm{S}$ & 1.468184 & -0.831029 & 0.059309 \\
$\mathrm{O}$ & 0.484636 & -1.875235 & 0.066577 \\
$\mathrm{O}$ & 2.792313 & -1.144199 & 0.484131 \\
$\mathrm{O}$ & 0.986023 & 0.329336 & 0.962810 \\
$\mathrm{O}$ & 1.471036 & -0.242944 & -1.352877 \\
$\mathrm{H}$ & -0.006715 & 0.468521 & 0.845621 \\
$\mathrm{H}$ & 2.206460 & 0.477690 & -1.459827 \\
$\mathrm{H}$ & 3.639001 & 1.561584 & -0.570564 \\
$\mathrm{H}$ & 3.990255 & 1.351775 & -2.080286 \\
$\mathrm{O}$ & 3.259810 & 1.521348 & -1.481262 \\
$\mathrm{O}$ & 4.086052 & 1.298603 & 1.045338 \\
$\mathrm{H}$ & 3.794474 & 0.376782 & 1.118226 \\
$\mathrm{H}$ & 3.565561 & 1.780809 & 1.691976 \\
\hline
\end{tabular}

Table S47. Cartesian coordinate of $(\mathbf{G W})_{1}(\mathbf{S A})_{1} \mathbf{W}_{3}$.

\begin{tabular}{|c|c|c|c|}
\hline Atoms & $X$ & $Y$ & $Z$ \\
\hline C & -2.225667 & -1.446542 & -0.420048 \\
O & -2.096585 & -2.499899 & 0.492785 \\
H & -3.191523 & -1.467009 & -0.927531 \\
C & -2.156773 & -0.145775 & 0.399125 \\
O & -2.398630 & 0.916753 & -0.106431 \\
O & -1.804924 & -0.254033 & 1.668856 \\
H & -1.575704 & -1.182730 & 1.846696 \\
O & -1.249272 & -1.459171 & -1.397920 \\
H & -1.835130 & -3.294126 & 0.017356 \\
H & -0.385072 & -1.386866 & -0.939113 \\
S & 2.101147 & -0.516820 & 0.074095 \\
O & 0.714103 & -0.886371 & 0.297420 \\
O & 3.096613 & -1.509797 & 0.189103 \\
\hline
\end{tabular}




\begin{tabular}{|l|r|r|r|}
\hline O & 2.430782 & 0.629374 & 1.042729 \\
$\mathrm{O}$ & 2.195552 & 0.103908 & -1.332512 \\
$\mathrm{H}$ & 1.599513 & 1.161173 & 1.295145 \\
$\mathrm{H}$ & 1.334061 & 0.563860 & -1.611496 \\
$\mathrm{O}$ & -0.007655 & 1.163422 & -2.077937 \\
$\mathrm{H}$ & -0.717249 & 0.508860 & -2.038968 \\
$\mathrm{H}$ & -0.326917 & 1.943811 & -1.600427 \\
$\mathrm{O}$ & 0.313752 & 1.877386 & 1.698999 \\
$\mathrm{H}$ & -0.051988 & 2.488338 & 1.040488 \\
$\mathrm{H}$ & -0.370808 & 1.214091 & 1.852828 \\
$\mathrm{O}$ & -1.029103 & 3.222872 & -0.380241 \\
$\mathrm{H}$ & -1.831936 & 2.705175 & -0.217285 \\
$\mathrm{H}$ & -1.288021 & 4.132879 & -0.536476 \\
\hline
\end{tabular}

Table S48. Cartesian coordinate of $(\mathbf{G W})_{1}(\mathbf{S A})_{1} \mathbf{A}_{1}$.

\begin{tabular}{|c|c|c|c|}
\hline Atoms & $X$ & $Y$ & $Z$ \\
\hline C & 1.494616 & -0.535948 & 0.461054 \\
O & 0.905402 & -1.253696 & -0.319672 \\
O & 0.975115 & 0.090826 & 1.472303 \\
H & -0.035131 & 0.029342 & 1.452954 \\
C & 2.977661 & -0.231696 & 0.246460 \\
H & 3.517989 & -0.235101 & 1.193384 \\
O & 3.561189 & -1.153131 & -0.586785 \\
O & 2.960988 & 1.069198 & -0.308592 \\
H & 3.851783 & 1.271576 & -0.609225 \\
H & 2.887306 & -1.473002 & -1.201504 \\
S & -2.227118 & -0.159579 & -0.027040 \\
O & -1.564742 & 0.181988 & 1.223185 \\
O & -3.636618 & -0.147496 & -0.042968 \\
\hline
\end{tabular}




\begin{tabular}{|l|l|l|l|}
\hline O & -1.772966 & -1.589332 & -0.431679 \\
O & -1.641488 & 0.727298 & -1.118262 \\
H & -0.788319 & -1.623396 & -0.430840 \\
H & -0.875212 & 1.402839 & -0.770365 \\
N & 0.125086 & 2.364446 & -0.296403 \\
H & -0.010220 & 3.266256 & -0.739265 \\
H & 1.093637 & 2.081655 & -0.435418 \\
H & -0.012236 & 2.482610 & 0.702415 \\
\hline
\end{tabular}

Table S49. Cartesian coordinate of $(\mathbf{G W})_{1}(\mathbf{S A})_{1} \mathbf{A}_{1} \mathbf{W}_{1}$.

\begin{tabular}{|c|c|c|c|}
\hline Atoms & $\mathrm{X}$ & $\mathrm{Y}$ & $\mathrm{Z}$ \\
\hline $\mathrm{C}$ & -1.604107 & -0.449216 & -0.636632 \\
$\mathrm{O}$ & -1.249343 & -1.574872 & -0.368255 \\
$\mathrm{O}$ & -0.880916 & 0.492561 & -1.161642 \\
$\mathrm{H}$ & 0.117160 & 0.226703 & -1.208701 \\
$\mathrm{C}$ & -3.021594 & 0.016161 & -0.296140 \\
$\mathrm{H}$ & -3.505126 & 0.454755 & -1.170579 \\
$\mathrm{O}$ & -3.791655 & -1.020515 & 0.163621 \\
$\mathrm{O}$ & -2.829459 & 1.019080 & 0.693029 \\
$\mathrm{H}$ & -3.684340 & 1.183890 & 1.101689 \\
$\mathrm{H}$ & -3.199299 & -1.718762 & 0.475401 \\
$\mathrm{~S}$ & 1.927461 & -0.716236 & 0.209122 \\
$\mathrm{O}$ & 1.570486 & 0.010494 & -1.039836 \\
$\mathrm{O}$ & 3.321597 & -0.756936 & 0.461057 \\
$\mathrm{O}$ & 1.468572 & -2.195821 & -0.030274 \\
$\mathrm{O}$ & 1.090222 & -0.216092 & 1.304812 \\
$\mathrm{H}$ & 0.501852 & -2.197229 & -0.174757 \\
$\mathrm{H}$ & 0.379427 & 1.139643 & 1.238130 \\
$\mathrm{~N}$ & -0.096348 & 2.097679 & 1.110694 \\
\hline & & & \\
\hline
\end{tabular}




\begin{tabular}{|l|l|l|l|}
\hline $\mathrm{H}$ & -0.058996 & 2.624414 & 1.976734 \\
$\mathrm{H}$ & -1.068672 & 1.944878 & 0.826394 \\
$\mathrm{H}$ & 0.444765 & 2.579410 & 0.370137 \\
$\mathrm{O}$ & 1.703342 & 2.754402 & -0.892229 \\
$\mathrm{H}$ & 2.539777 & 3.220686 & -0.927632 \\
$\mathrm{H}$ & 1.899949 & 1.817539 & -1.073256 \\
\hline
\end{tabular}

Table S50. Cartesian coordinate of $(\mathbf{G W})_{1}(\mathbf{S A})_{1} \mathbf{A}_{1} \mathbf{W}_{2}$.

\begin{tabular}{|c|c|c|c|}
\hline Atoms & $x$ & $Y$ & Z \\
\hline$C$ & 2.037073 & -0.331718 & 0.553558 \\
\hline 0 & 1.855291 & -1.469000 & 0.183091 \\
\hline 0 & 1.217000 & 0.401312 & 1.246803 \\
\hline $\mathrm{H}$ & 0.299282 & -0.056802 & 1.333466 \\
\hline C & 3.313069 & 0.419190 & 0.167945 \\
\hline $\mathrm{H}$ & 3.814000 & 0.804847 & 1.058074 \\
\hline 0 & 4.183569 & -0.394392 & -0.512133 \\
\hline 0 & 2.859787 & 1.503305 & -0.629018 \\
\hline $\mathrm{H}$ & 3.628942 & 1.861403 & -1.081691 \\
\hline $\mathrm{H}$ & 3.682408 & -1.144529 & -0.860491 \\
\hline$S$ & -1.397625 & -1.124758 & -0.101480 \\
\hline 0 & -1.089688 & -0.575554 & 1.234977 \\
\hline 0 & -2.799681 & -1.320339 & -0.315681 \\
\hline 0 & -0.758297 & -2.555033 & -0.134025 \\
\hline 0 & -0.720847 & -0.352638 & -1.137151 \\
\hline $\mathrm{H}$ & 0.206331 & -2.448132 & -0.021623 \\
\hline $\mathrm{H}$ & -0.274224 & 1.189710 & -0.989448 \\
\hline$N$ & -0.050784 & 2.177167 & -0.689722 \\
\hline $\mathrm{H}$ & -0.236683 & 2.828072 & -1.444942 \\
\hline $\mathrm{H}$ & 0.933803 & 2.210923 & -0.415982 \\
\hline
\end{tabular}




\begin{tabular}{|l|l|l|l|}
\hline $\mathrm{H}$ & -0.706734 & 2.349873 & 0.120485 \\
$\mathrm{O}$ & -1.972676 & 2.178803 & 1.198368 \\
$\mathrm{H}$ & -1.798362 & 1.315612 & 1.598870 \\
$\mathrm{H}$ & -2.738260 & 1.999696 & 0.618664 \\
$\mathrm{O}$ & -3.763474 & 1.287478 & -0.691362 \\
$\mathrm{H}$ & -4.719595 & 1.353849 & -0.678100 \\
$\mathrm{H}$ & -3.562145 & 0.335074 & -0.665502 \\
\hline
\end{tabular}

Table S51. Cartesian coordinate of $(\mathbf{G W})_{1}(\mathbf{S A})_{1} \mathbf{A}_{1} \mathbf{W}_{3}$.

\begin{tabular}{|c|c|c|c|}
\hline Atoms & $x$ & $Y$ & Z \\
\hline C & -2.124681 & 0.177631 & 0.499189 \\
\hline 0 & -2.150828 & 1.322678 & 0.106635 \\
\hline 0 & -1.167167 & -0.408980 & 1.143533 \\
\hline $\mathrm{H}$ & -0.309522 & 0.163412 & 1.217019 \\
\hline C & -3.305177 & -0.756302 & 0.212701 \\
\hline $\mathrm{H}$ & -3.637505 & -1.243702 & 1.130822 \\
\hline 0 & -4.360179 & -0.068337 & -0.332112 \\
\hline 0 & -2.791890 & -1.732123 & -0.683614 \\
\hline $\mathrm{H}$ & -3.549015 & -2.172167 & -1.081464 \\
\hline $\mathrm{H}$ & -4.015208 & 0.757343 & -0.699495 \\
\hline$S$ & 1.715990 & 0.859768 & -0.045559 \\
\hline 0 & 1.044187 & 0.746524 & 1.267167 \\
\hline 0 & 2.990872 & 0.186476 & -0.062438 \\
\hline 0 & 2.021467 & 2.359681 & -0.258105 \\
\hline 0 & 0.805179 & 0.435701 & -1.106458 \\
\hline $\mathrm{H}$ & 1.150363 & 2.862729 & -0.254808 \\
\hline $\mathrm{H}$ & 0.352274 & -1.172692 & -0.991270 \\
\hline $\mathrm{N}$ & 0.133624 & -2.155847 & -0.694302 \\
\hline $\mathrm{H}$ & 0.632464 & -2.798678 & -1.302666 \\
\hline
\end{tabular}




\begin{tabular}{|l|l|l|l|}
\hline $\mathrm{H}$ & -0.879872 & -2.288053 & -0.690886 \\
$\mathrm{H}$ & 0.534958 & -2.244133 & 0.267404 \\
$\mathrm{O}$ & 1.612194 & -2.108748 & 1.630872 \\
$\mathrm{H}$ & 1.649287 & -1.167771 & 1.853495 \\
$\mathrm{H}$ & 2.432552 & -2.282637 & 1.152170 \\
$\mathrm{O}$ & 2.916975 & -2.393609 & -0.941194 \\
$\mathrm{H}$ & 3.718564 & -2.699342 & -1.368225 \\
$\mathrm{H}$ & 3.046615 & -1.437808 & -0.783161 \\
$\mathrm{O}$ & -0.344385 & 3.426977 & -0.258729 \\
$\mathrm{H}$ & -0.610387 & 3.712962 & -1.135422 \\
$\mathrm{H}$ & -0.939033 & 2.687285 & -0.040245 \\
\hline
\end{tabular}

Table S52. Cartesian coordinate of $(\mathbf{G W})_{1}(\mathbf{S A})_{1} \mathbf{A}_{1} \mathbf{W}_{4}$.

\begin{tabular}{|c|c|c|c|}
\hline Atoms & $x$ & $Y$ & Z \\
\hline$C$ & 2.099070 & -0.786610 & 0.589820 \\
\hline 0 & 1.993017 & -1.583289 & -0.319777 \\
\hline 0 & 1.323680 & -0.706451 & 1.634160 \\
\hline $\mathrm{H}$ & 0.490555 & -1.261844 & 1.502324 \\
\hline C & 3.153999 & 0.324476 & 0.540727 \\
\hline $\mathrm{H}$ & 3.639525 & 0.432556 & 1.509259 \\
\hline 0 & 4.089270 & 0.048981 & -0.431019 \\
\hline 0 & 2.465182 & 1.529758 & 0.309014 \\
\hline $\mathrm{H}$ & 2.144330 & 1.530470 & -0.613201 \\
\hline $\mathrm{H}$ & 3.722927 & -0.636292 & -1.005141 \\
\hline$S$ & -1.220662 & -1.366799 & -0.305046 \\
\hline 0 & -0.919852 & -1.835531 & 1.051896 \\
\hline 0 & -2.611900 & -1.451486 & -0.654816 \\
\hline 0 & -0.485471 & -2.346647 & -1.286246 \\
\hline 0 & -0.648884 & -0.039306 & -0.520472 \\
\hline
\end{tabular}




\begin{tabular}{|l|l|l|l|}
\hline $\mathrm{H}$ & 0.461780 & -2.347360 & -1.041257 \\
$\mathrm{H}$ & -0.639655 & 2.526537 & -1.640568 \\
$\mathrm{~N}$ & -0.201581 & 2.080256 & 1.369097 \\
$\mathrm{H}$ & -0.078533 & 2.829979 & 2.041556 \\
$\mathrm{H}$ & 0.706344 & 1.637907 & 1.180340 \\
$\mathrm{H}$ & -0.898466 & 1.385257 & 1.724843 \\
$\mathrm{O}$ & -2.305486 & 0.492203 & 2.160231 \\
$\mathrm{H}$ & -2.088125 & -0.446725 & 2.086674 \\
$\mathrm{H}$ & -2.886637 & 0.679115 & 1.406618 \\
$\mathrm{O}$ & -3.447440 & 1.141280 & -0.419740 \\
$\mathrm{H}$ & -4.362932 & 1.316586 & -0.646061 \\
$\mathrm{H}$ & -3.265442 & 0.217535 & -0.682512 \\
$\mathrm{O}$ & -1.271216 & 2.879496 & -0.999810 \\
$\mathrm{H}$ & -2.026929 & 2.267699 & -0.998883 \\
$\mathrm{H}$ & -0.580444 & 2.459047 & 0.460296 \\
$\mathrm{O}$ & 0.991647 & 1.345514 & -2.046903 \\
$\mathrm{H}$ & 1.285498 & 1.048617 & -2.910118 \\
$\mathrm{H}$ & 0.419069 & 0.644521 & -1.672539 \\
\hline & & & \\
\hline
\end{tabular}

Table S53. Cartesian coordinate of $(\mathbf{G W})_{1}(\mathbf{S A})_{1} \mathbf{A}_{1} \mathbf{W}_{5}$.

\begin{tabular}{|c|c|c|c|}
\hline Atoms & $\mathrm{X}$ & $\mathrm{Y}$ & $\mathrm{Z}$ \\
\hline $\mathrm{C}$ & 2.174590 & -1.104255 & 0.519043 \\
$\mathrm{O}$ & 2.066035 & -1.634525 & -0.567011 \\
$\mathrm{O}$ & 1.308911 & -1.174688 & 1.494313 \\
$\mathrm{H}$ & 0.444469 & -1.575631 & 1.171537 \\
$\mathrm{C}$ & 3.341702 & -0.162991 & 0.832018 \\
$\mathrm{H}$ & 3.729742 & -0.356142 & 1.830818 \\
$\mathrm{O}$ & 4.343761 & -0.320765 & -0.100307 \\
$\mathrm{O}$ & 2.811750 & 1.138117 & 0.856602 \\
\hline
\end{tabular}




\begin{tabular}{|c|c|c|c|}
\hline $\mathrm{H}$ & 2.605046 & 1.392221 & -0.061481 \\
\hline $\mathrm{H}$ & 3.966799 & -0.772405 & -0.866126 \\
\hline$S$ & -1.087543 & -1.022259 & -0.733546 \\
\hline 0 & -0.959571 & -1.854309 & 0.465467 \\
\hline 0 & -2.447090 & -0.845909 & -1.177935 \\
\hline 0 & -0.381405 & -1.817027 & -1.891047 \\
\hline 0 & -0.358446 & 0.230707 & -0.582083 \\
\hline $\mathrm{H}$ & 0.530796 & -2.006987 & -1.594220 \\
\hline $\mathrm{H}$ & 0.331696 & 2.952456 & -1.144640 \\
\hline $\mathrm{N}$ & 0.015065 & 1.754044 & 1.733631 \\
\hline $\mathrm{H}$ & 0.204835 & 2.305892 & 2.563487 \\
\hline $\mathrm{H}$ & 0.775635 & 1.086820 & 1.584841 \\
\hline $\mathrm{H}$ & -0.919416 & 1.275810 & 1.810632 \\
\hline 0 & -2.564878 & 0.988374 & 1.738012 \\
\hline $\mathrm{H}$ & -2.972394 & 0.101149 & 1.757585 \\
\hline $\mathrm{H}$ & -2.827578 & 1.372007 & 0.888773 \\
\hline 0 & -2.834400 & 1.904008 & -0.975389 \\
\hline $\mathrm{H}$ & -3.610061 & 2.246029 & -1.423519 \\
\hline $\mathrm{H}$ & -2.740067 & 0.971418 & -1.242572 \\
\hline $\mathrm{O}$ & -0.357410 & 3.243884 & -0.533239 \\
\hline $\mathrm{H}$ & -1.175534 & 2.817192 & -0.838131 \\
\hline $\mathrm{H}$ & -0.047933 & 2.374777 & 0.886811 \\
\hline 0 & 1.683758 & 1.555348 & -1.669309 \\
\hline $\mathrm{H}$ & 2.063037 & 1.330230 & -2.520614 \\
\hline $\mathrm{H}$ & 0.966009 & 0.916767 & -1.488365 \\
\hline 0 & -3.619451 & -1.571510 & 1.472526 \\
\hline $\mathrm{H}$ & -3.907737 & -1.496064 & 0.556782 \\
\hline $\mathrm{H}$ & -2.752375 & -1.987861 & 1.378568 \\
\hline
\end{tabular}


Table S54. Cartesian coordinate of $(\mathbf{G W})_{1} \mathbf{A}_{2}$.

\begin{tabular}{|c|c|c|c|}
\hline Atoms & $\mathrm{X}$ & $\mathrm{Y}$ & $\mathrm{Z}$ \\
\hline $\mathrm{C}$ & -0.754971 & 0.119275 & -0.147947 \\
$\mathrm{O}$ & -1.248187 & 1.106531 & 0.346824 \\
$\mathrm{O}$ & -1.416429 & -0.929907 & -0.568306 \\
$\mathrm{H}$ & -2.393714 & -0.798866 & -0.370441 \\
$\mathrm{C}$ & 0.760825 & -0.004814 & -0.310944 \\
$\mathrm{H}$ & 1.000281 & -0.442659 & -1.283548 \\
$\mathrm{O}$ & 1.335971 & 1.266553 & -0.283483 \\
$\mathrm{O}$ & 1.183123 & -0.818783 & 0.730921 \\
$\mathrm{H}$ & 2.144487 & -0.938542 & 0.611217 \\
$\mathrm{H}$ & 0.783099 & 1.810276 & 0.293301 \\
$\mathrm{~N}$ & -3.933884 & -0.253910 & 0.138630 \\
$\mathrm{H}$ & -3.581304 & 0.632959 & 0.487963 \\
$\mathrm{H}$ & -4.369502 & -0.744467 & 0.909788 \\
$\mathrm{H}$ & -4.650263 & -0.060453 & -0.549908 \\
$\mathrm{~N}$ & 3.891855 & -0.403739 & 0.017518 \\
$\mathrm{H}$ & 3.540276 & 0.528205 & -0.182871 \\
$\mathrm{H}$ & -362304 & -0.745299 & -0.810889 \\
$\mathrm{H}$ & -0.319527 & 0.748055 \\
\hline & & & \\
\hline
\end{tabular}

Table S55. Cartesian coordinate of $(\mathbf{G W})_{1} \mathbf{A}_{2} \mathbf{W}_{1}$.

\begin{tabular}{|c|c|c|c|}
\hline Atoms & $X$ & $Y$ & $Z$ \\
\hline C & -0.242799 & -0.138444 & -0.223019 \\
O & -0.840886 & 0.899910 & -0.020134 \\
O & -0.774692 & -1.312397 & -0.365598 \\
H & -1.795213 & -1.302022 & -0.235925 \\
C & 1.285479 & -0.135635 & -0.304062 \\
H & 1.620450 & -0.814611 & -1.091973 \\
\hline
\end{tabular}




\begin{tabular}{|l|l|l|l|}
\hline O & 1.738406 & 1.138514 & -0.648796 \\
O & 1.721372 & -0.550013 & 0.946613 \\
H & 2.694325 & -0.608255 & 0.889242 \\
H & 1.130413 & 1.770784 & -0.244446 \\
N & -3.396030 & -1.376221 & -0.016920 \\
H & -3.725397 & -0.438660 & 0.215617 \\
H & -3.650551 & -1.998509 & 0.740538 \\
H & -3.895440 & -1.683659 & -0.842774 \\
N & 4.415146 & -0.145066 & 0.178297 \\
H & 4.016545 & 0.655667 & -0.303389 \\
H & 4.922030 & -0.697361 & -0.501766 \\
H & 5.089583 & 0.203692 & 0.847937 \\
O & -3.538636 & 1.517158 & 0.276110 \\
H & -2.577472 & 1.407229 & 0.179519 \\
H & -3.663672 & 2.253803 & 0.874711 \\
\hline
\end{tabular}

Table S56. Cartesian coordinate of $(\mathbf{G W})_{1}(\mathbf{S A})_{2}$.

\begin{tabular}{|c|c|c|c|}
\hline Atoms & $\mathrm{X}$ & $\mathrm{Y}$ & $\mathrm{Z}$ \\
\hline $\mathrm{C}$ & -0.170884 & 2.445085 & -0.394854 \\
$\mathrm{O}$ & -1.194486 & 3.198631 & -0.965419 \\
$\mathrm{H}$ & 0.667685 & 3.072997 & -0.083058 \\
$\mathrm{C}$ & -0.769623 & 1.793141 & 0.865421 \\
$\mathrm{O}$ & -0.109944 & 1.044520 & 1.555163 \\
$\mathrm{O}$ & -2.002212 & 2.083757 & 1.164312 \\
$\mathrm{H}$ & -2.389463 & 2.621060 & 0.453423 \\
$\mathrm{O}$ & 0.338682 & 1.486323 & -1.256843 \\
$\mathrm{H}$ & -0.921755 & 3.519340 & -1.829688 \\
$\mathrm{H}$ & -0.297462 & 0.741064 & -1.306298 \\
$\mathrm{~S}$ & -1.967289 & -1.465979 & -0.196178 \\
\hline
\end{tabular}




\begin{tabular}{|l|l|l|l|}
\hline O & -1.655864 & -0.193504 & -0.799471 \\
$\mathrm{O}$ & -3.258555 & -2.000981 & -0.343660 \\
$\mathrm{O}$ & -1.657546 & -1.349947 & 1.326489 \\
$\mathrm{O}$ & -0.961734 & -2.524463 & -0.701240 \\
$\mathrm{H}$ & -1.016947 & -0.638604 & 1.517249 \\
$\mathrm{H}$ & -0.038165 & -2.208920 & -0.576072 \\
$\mathrm{~S}$ & 2.536756 & -0.596696 & 0.053299 \\
$\mathrm{O}$ & 1.236256 & -1.200720 & -0.071018 \\
$\mathrm{O}$ & 3.682712 & -1.393842 & 0.215893 \\
$\mathrm{O}$ & 2.447716 & 0.414816 & 1.224435 \\
$\mathrm{O}$ & 2.768399 & 0.305291 & -1.191230 \\
$\mathrm{H}$ & 1.504068 & 0.673132 & 1.384955 \\
$\mathrm{H}$ & 1.956223 & 0.834316 & -1.357148 \\
\hline
\end{tabular}

Table S57. Cartesian coordinate of $(\mathbf{G W})_{1}(\mathbf{S A})_{2} \mathbf{W}_{1}$.

\begin{tabular}{|c|c|c|c|}
\hline Atoms & $X$ & $Y$ & $Z$ \\
\hline C & -3.000065 & -1.492552 & 0.096239 \\
O & -3.307766 & -0.368227 & -0.198411 \\
O & -3.828823 & -2.365453 & 0.640072 \\
H & -4.689459 & -1.943503 & 0.774592 \\
C & -1.588157 & -2.056006 & -0.110279 \\
H & -1.610803 & -3.143390 & -0.068544 \\
O & -1.078008 & -1.691201 & -1.328335 \\
O & -0.854548 & -1.534351 & 0.975131 \\
H & 0.100849 & -1.704644 & 0.841844 \\
H & -0.973024 & -0.727479 & -1.352820 \\
S & 0.017590 & 1.725896 & 0.252579 \\
O & -0.026040 & 0.920619 & -0.952396 \\
O & 1.314126 & 2.081575 & 0.724493 \\
\hline
\end{tabular}




\begin{tabular}{|l|l|l|l|}
\hline O & -0.798707 & 1.006826 & 1.335689 \\
O & -0.770116 & 3.009334 & 0.050893 \\
H & -0.818327 & -0.001306 & 1.206503 \\
H & -1.704297 & 2.794572 & -0.324469 \\
S & 2.869147 & -0.777293 & -0.068595 \\
O & 1.725757 & -1.058963 & 0.756107 \\
O & 3.878111 & -1.740925 & -0.246028 \\
O & 3.535336 & 0.511542 & 0.479010 \\
O & 2.348228 & -0.363724 & -1.475052 \\
H & 2.843367 & 1.174138 & 0.695511 \\
H & 1.487165 & 0.102868 & -1.399791 \\
O & -3.016936 & 2.317227 & -0.858946 \\
H & -3.150069 & 1.379779 & -0.637853 \\
$\mathrm{H}$ & -3.108791 & 2.388439 & -1.812282 \\
\hline
\end{tabular}

Table S58. Cartesian coordinate of $(\mathbf{G W})_{1}(\mathbf{S A})_{2} \mathbf{W}_{2}$.

\begin{tabular}{|c|c|c|c|}
\hline Atoms & $\mathrm{X}$ & $\mathrm{Y}$ & $\mathrm{Z}$ \\
\hline $\mathrm{C}$ & -0.058462 & 2.492284 & -0.593307 \\
$\mathrm{O}$ & -1.292508 & 2.930556 & -1.055362 \\
$\mathrm{H}$ & 0.669104 & 3.303505 & -0.518597 \\
$\mathrm{C}$ & -0.292828 & 1.947442 & 0.829343 \\
$\mathrm{O}$ & 0.632246 & 1.554327 & 1.503999 \\
$\mathrm{O}$ & -1.512102 & 1.952667 & 1.293458 \\
$\mathrm{H}$ & -2.147561 & 2.179519 & 0.590299 \\
$\mathrm{O}$ & 0.502714 & 1.522377 & -1.419329 \\
$\mathrm{H}$ & -1.238471 & 3.131042 & -1.994077 \\
$\mathrm{H}$ & -0.071501 & 0.728455 & -1.352499 \\
$\mathrm{~S}$ & -2.463014 & -0.679892 & -0.198789 \\
$\mathrm{O}$ & -1.101763 & -0.311782 & -0.529731 \\
\hline
\end{tabular}




\begin{tabular}{|l|l|l|l|}
\hline O & -3.477520 & 0.286144 & -0.407041 \\
\hline O & -2.468274 & -1.157148 & 1.249765 \\
H & -2.813306 & -1.936712 & -1.024584 \\
H & -1.508517 & -1.188321 & 1.652413 \\
S & -2.016179 & -2.531069 & -1.014949 \\
O & 2.917522 & -0.507440 & -0.131530 \\
O & 1.627001 & -1.150525 & -0.072303 \\
O & 4.089625 & -1.284671 & -0.155253 \\
O & 3.009759 & 0.473771 & 1.063743 \\
H & 2.929967 & 0.416416 & -1.376626 \\
H & 2.151909 & 0.946714 & 1.212558 \\
O & 2.072102 & 0.904270 & -1.453150 \\
H & -0.147966 & -1.140134 & 2.183496 \\
H & 0.023474 & -0.235010 & 2.473960 \\
O & 0.464568 & -1.268510 & 1.439771 \\
H & -0.450828 & -3.065041 & -0.721779 \\
H & 0.256568 & -2.404863 & -0.716565 \\
\hline & -3.928724 & -0.709901 \\
\hline
\end{tabular}

Table S59. Cartesian coordinate of $(\mathbf{G W})_{1}(\mathbf{S A})_{2} \mathbf{W}_{3}$.

\begin{tabular}{|c|c|c|c|}
\hline Atoms & $\mathrm{X}$ & $\mathrm{Y}$ & $\mathrm{Z}$ \\
\hline $\mathrm{C}$ & -0.111444 & -2.447931 & -0.643890 \\
$\mathrm{O}$ & 1.030228 & -3.072018 & -1.131503 \\
$\mathrm{H}$ & -0.960707 & -3.132663 & -0.609648 \\
$\mathrm{C}$ & 0.141037 & -1.984425 & 0.804577 \\
$\mathrm{O}$ & -0.827495 & -1.661337 & 1.476650 \\
$\mathrm{O}$ & 1.325736 & -1.953715 & 1.295104 \\
$\mathrm{H}$ & 2.126574 & -2.049859 & 0.653510 \\
$\mathrm{O}$ & -0.504617 & -1.375372 & -1.448547 \\
\hline
\end{tabular}




\begin{tabular}{|c|c|c|c|}
\hline $\mathrm{H}$ & 0.826828 & -3.449141 & -1.992486 \\
\hline $\mathrm{H}$ & 0.131576 & -0.639874 & -1.329380 \\
\hline$S$ & 2.277175 & 1.275409 & -0.181659 \\
\hline 0 & 1.136871 & 0.456168 & -0.524710 \\
\hline 0 & 3.570507 & 0.766072 & -0.497746 \\
\hline $\mathrm{O}$ & 2.200731 & 1.581925 & 1.307091 \\
\hline 0 & 2.133604 & 2.627599 & -0.886846 \\
\hline $\mathrm{H}$ & 1.284648 & 1.298180 & 1.737568 \\
\hline $\mathrm{H}$ & 1.163922 & 2.928354 & -0.845842 \\
\hline$S$ & -3.072044 & 0.350474 & -0.101486 \\
\hline 0 & -1.833961 & 1.034484 & 0.214180 \\
\hline 0 & -4.279707 & 1.072290 & -0.089446 \\
\hline 0 & -3.188933 & -0.853472 & 0.851540 \\
\hline 0 & -2.909828 & -0.283372 & -1.504847 \\
\hline $\mathrm{H}$ & -2.285584 & -1.231875 & 1.083303 \\
\hline $\mathrm{H}$ & -2.029248 & -0.737558 & -1.570794 \\
\hline 0 & 0.036091 & 0.916711 & 2.317736 \\
\hline $\mathrm{H}$ & 0.041597 & -0.015193 & 2.572792 \\
\hline $\mathrm{H}$ & -0.664701 & 0.970784 & 1.644787 \\
\hline 0 & -0.352465 & 3.187882 & -0.752386 \\
\hline $\mathrm{H}$ & -0.909156 & 2.425548 & -0.518512 \\
\hline $\mathrm{H}$ & -0.703039 & 3.944248 & -0.277713 \\
\hline 0 & 3.418349 & -1.986145 & -0.098489 \\
\hline $\mathrm{H}$ & 3.201293 & -2.335365 & -0.966975 \\
\hline $\mathrm{H}$ & 3.675424 & -1.057167 & -0.236648 \\
\hline
\end{tabular}

Table S60. Cartesian coordinate of $(\mathbf{G W})_{1}(\mathbf{S A})_{2} \mathbf{W}_{4}$.

\begin{tabular}{|c|c|c|c|}
\hline Atoms & $X$ & $Y$ & $Z$ \\
\hline$C$ & 0.255851 & -2.263394 & -0.569567 \\
\hline
\end{tabular}




\begin{tabular}{|c|c|c|c|}
\hline 0 & 1.456077 & -2.391871 & -1.235809 \\
\hline $\mathrm{H}$ & -0.165064 & -3.264804 & -0.469046 \\
\hline$C$ & 0.421697 & -1.711557 & 0.866139 \\
\hline $\mathrm{O}$ & -0.537797 & -1.341776 & 1.516342 \\
\hline 0 & 1.605283 & -1.695813 & 1.408718 \\
\hline $\mathrm{H}$ & 2.418735 & -1.879818 & 0.823793 \\
\hline $\mathrm{O}$ & -0.680060 & -1.487684 & -1.253161 \\
\hline $\mathrm{H}$ & 1.813289 & -1.516632 & -1.465910 \\
\hline $\mathrm{H}$ & -0.396578 & -0.557622 & -1.165366 \\
\hline$S$ & 2.087097 & 1.059798 & -0.647069 \\
\hline 0 & 0.722344 & 0.614426 & -0.384702 \\
\hline $\mathrm{O}$ & 2.857036 & 0.131210 & -1.442588 \\
\hline $\mathrm{O}$ & 2.763921 & 1.416637 & 0.602013 \\
\hline 0 & 1.963301 & 2.358308 & -1.499317 \\
\hline $\mathrm{H}$ & 2.023843 & 1.103209 & 1.798725 \\
\hline $\mathrm{H}$ & 1.109889 & 2.795330 & -1.275901 \\
\hline$S$ & -3.323268 & -0.087470 & -0.172544 \\
\hline 0 & -2.211316 & 0.837788 & -0.228112 \\
\hline $\mathrm{O}$ & -4.631866 & 0.415369 & -0.048120 \\
\hline 0 & -3.040626 & -1.074126 & 0.975452 \\
\hline $\mathrm{O}$ & -3.278979 & -0.967662 & -1.450085 \\
\hline $\mathrm{H}$ & -2.053703 & -1.219706 & 1.124069 \\
\hline $\mathrm{H}$ & -2.376256 & -1.351987 & -1.540820 \\
\hline $\mathrm{O}$ & 1.390268 & 0.864931 & 2.594980 \\
\hline $\mathrm{H}$ & 1.317192 & -0.104848 & 2.595840 \\
\hline $\mathrm{H}$ & 0.442945 & 1.279156 & 2.337943 \\
\hline $\mathrm{O}$ & -0.535389 & 3.125797 & -0.678988 \\
\hline $\mathrm{H}$ & -1.052689 & 2.312319 & -0.798155 \\
\hline $\mathrm{H}$ & -1.083997 & 3.845196 & -1.001237 \\
\hline
\end{tabular}




\begin{tabular}{|l|l|l|l|}
\hline O & -0.740185 & 1.911933 & 1.929470 \\
H & -1.271216 & 1.302906 & 1.388182 \\
H & -0.535431 & 2.623896 & 1.304284 \\
O & 3.862219 & -1.887709 & 0.240000 \\
H & 3.873306 & -1.089867 & -0.314558 \\
H & 3.935331 & -2.622354 & -0.376226 \\
\hline
\end{tabular}

Table S61. Cartesian coordinate of $(\mathbf{G W})_{1}(\mathbf{S A})_{2} \mathbf{W}_{5}$.

\begin{tabular}{|c|c|c|c|}
\hline Atoms & $x$ & $Y$ & Z \\
\hline C & 0.677070 & -2.410441 & 0.015926 \\
\hline 0 & 1.806521 & -2.475771 & -0.778208 \\
\hline $\mathrm{H}$ & 0.526532 & -3.410897 & 0.425819 \\
\hline C & 0.866236 & -1.467907 & 1.222662 \\
\hline 0 & -0.104361 & -0.962278 & 1.785063 \\
\hline 0 & 2.043712 & -1.253057 & 1.685647 \\
\hline $\mathrm{H}$ & 2.863911 & -1.584274 & 1.160205 \\
\hline 0 & -0.482879 & -2.076087 & -0.670420 \\
\hline $\mathrm{H}$ & 2.001854 & -1.598982 & -1.144675 \\
\hline $\mathrm{H}$ & -0.464626 & -1.129720 & -0.894349 \\
\hline$S$ & 2.215893 & 1.020214 & -0.747453 \\
\hline 0 & 0.914604 & 0.368244 & -0.654134 \\
\hline 0 & 3.267539 & 0.142041 & -1.199864 \\
\hline 0 & 2.544806 & 1.711258 & 0.502719 \\
\hline $\mathrm{O}$ & 2.086327 & 2.112283 & -1.855682 \\
\hline $\mathrm{H}$ & 1.575904 & 1.621290 & 1.540012 \\
\hline $\mathrm{H}$ & 1.177644 & 2.485159 & -1.817152 \\
\hline$S$ & -2.978677 & 0.201052 & 0.088560 \\
\hline 0 & -1.925712 & 0.312824 & -0.891375 \\
\hline 0 & -3.419012 & 1.417202 & 0.688243 \\
\hline
\end{tabular}




\begin{tabular}{|c|c|c|c|}
\hline 0 & -2.557752 & -0.821099 & 1.155672 \\
\hline 0 & -4.193689 & -0.494366 & -0.526819 \\
\hline $\mathrm{H}$ & -1.556034 & -0.898239 & 1.313226 \\
\hline $\mathrm{H}$ & -3.889394 & -1.395230 & -0.889923 \\
\hline 0 & 0.800923 & 1.564234 & 2.252143 \\
\hline $\mathrm{H}$ & 0.509505 & 0.626352 & 2.294811 \\
\hline $\mathrm{H}$ & 0.000866 & 2.121052 & 1.891909 \\
\hline 0 & -0.552958 & 2.688981 & -1.469851 \\
\hline $\mathrm{H}$ & -0.880867 & 1.776380 & -1.358088 \\
\hline $\mathrm{H}$ & -1.147358 & 3.117404 & -2.090683 \\
\hline 0 & -1.076448 & 2.910544 & 1.286710 \\
\hline $\mathrm{H}$ & -1.963346 & 2.516166 & 1.289856 \\
\hline $\mathrm{H}$ & -0.872331 & 3.050688 & 0.346867 \\
\hline 0 & 4.232304 & -1.763126 & 0.540442 \\
\hline $\mathrm{H}$ & 4.216321 & -0.994206 & -0.057710 \\
\hline $\mathrm{H}$ & 4.161702 & -2.524945 & -0.042919 \\
\hline 0 & -3.134031 & -2.696359 & -1.300465 \\
\hline $\mathrm{H}$ & -2.203871 & -2.627670 & -1.017690 \\
\hline $\mathrm{H}$ & -3.110861 & -2.904253 & -2.237328 \\
\hline
\end{tabular}

Table S62. Cartesian coordinate of $(\mathbf{G W})_{1}(\mathbf{S A})_{2} \mathbf{W}_{6}$.

\begin{tabular}{|c|c|c|c|}
\hline Atoms & $X$ & $Y$ & $Z$ \\
\hline C & -0.478912 & 2.385900 & -0.117952 \\
O & -1.590565 & 2.404655 & -0.941379 \\
H & -0.342162 & 3.406280 & 0.244459 \\
C & -0.694188 & 1.496386 & 1.125518 \\
O & 0.268632 & 1.001971 & 1.721766 \\
O & -1.875887 & 1.297188 & 1.560863 \\
H & -2.716208 & 1.722260 & 1.063319 \\
\hline
\end{tabular}




\begin{tabular}{|c|c|c|c|}
\hline 0 & 0.698256 & 2.026775 & -0.763587 \\
\hline $\mathrm{H}$ & -1.802796 & 1.509029 & -1.252601 \\
\hline $\mathrm{H}$ & 0.683670 & 1.072245 & -0.949749 \\
\hline$S$ & -1.870575 & -1.090786 & -0.816099 \\
\hline 0 & -0.567374 & -0.477742 & -0.614604 \\
\hline $\mathrm{O}$ & -2.833305 & -0.211331 & -1.441078 \\
\hline $\mathrm{O}$ & -2.379416 & -1.674794 & 0.431232 \\
\hline $\mathrm{O}$ & -1.657483 & -2.267797 & -1.818986 \\
\hline $\mathrm{H}$ & -1.419728 & -1.550709 & 1.556876 \\
\hline $\mathrm{H}$ & -0.724374 & -2.576151 & -1.749930 \\
\hline$S$ & 3.222084 & -0.166620 & 0.162614 \\
\hline $\mathrm{O}$ & 2.245170 & -0.321708 & -0.888188 \\
\hline $\mathrm{O}$ & 3.632500 & -1.362224 & 0.823818 \\
\hline 0 & 2.719225 & 0.876270 & 1.167453 \\
\hline $\mathrm{O}$ & 4.468431 & 0.529993 & -0.388311 \\
\hline $\mathrm{H}$ & 1.704393 & 0.936006 & 1.288122 \\
\hline $\mathrm{H}$ & 4.169305 & 1.412759 & -0.794631 \\
\hline 0 & -0.670707 & -1.463600 & 2.267138 \\
\hline $\mathrm{H}$ & -0.377707 & -0.520128 & 2.264356 \\
\hline $\mathrm{H}$ & 0.146105 & -2.027650 & 1.935896 \\
\hline $\mathrm{O}$ & 0.973839 & -2.739571 & -1.389983 \\
\hline $\mathrm{H}$ & 1.325008 & -1.830092 & -1.316178 \\
\hline $\mathrm{H}$ & 1.586332 & -3.227839 & -1.945327 \\
\hline $\mathrm{O}$ & 1.249087 & -2.798781 & 1.401503 \\
\hline $\mathrm{H}$ & 2.133901 & -2.402048 & 1.452111 \\
\hline $\mathrm{H}$ & 1.111608 & -2.964029 & 0.452843 \\
\hline 0 & -3.962105 & 2.161427 & 0.600712 \\
\hline $\mathrm{H}$ & -4.510337 & 1.347476 & 0.466833 \\
\hline $\mathrm{H}$ & -3.791114 & 2.535673 & -0.269384 \\
\hline
\end{tabular}




\begin{tabular}{|l|l|l|l|}
\hline O & 3.379697 & 2.679230 & -1.277468 \\
H & 2.440058 & 2.583211 & -1.036551 \\
H & 3.392048 & 2.863266 & -2.219442 \\
O & -5.125262 & -0.192909 & 0.166289 \\
H & -4.859219 & -0.818689 & 0.846347 \\
H & -4.516165 & -0.382516 & -0.564534 \\
\hline
\end{tabular}

Table S63. Cartesian coordinate of $(\mathbf{G W})_{1}(\mathbf{S A})_{2} \mathbf{W}_{7}$.

\begin{tabular}{|c|c|c|c|}
\hline Atoms & $x$ & $Y$ & Z \\
\hline$C$ & -0.317957 & 2.408380 & -0.152723 \\
\hline 0 & -1.367569 & 2.460594 & -1.061997 \\
\hline $\mathrm{H}$ & -0.211981 & 3.412293 & 0.259917 \\
\hline C & -0.638706 & 1.446382 & 1.017323 \\
\hline 0 & 0.338820 & 0.868842 & 1.590867 \\
\hline 0 & -1.815632 & 1.298669 & 1.351875 \\
\hline $\mathrm{H}$ & -3.084353 & 2.284549 & 0.644423 \\
\hline 0 & 0.898015 & 2.085875 & -0.752057 \\
\hline $\mathrm{H}$ & -1.495446 & 1.565435 & -1.407852 \\
\hline $\mathrm{H}$ & 0.933511 & 1.132394 & -0.936653 \\
\hline$S$ & -1.741059 & -1.232237 & -1.082696 \\
\hline 0 & -0.601499 & -0.340333 & -1.006029 \\
\hline 0 & -2.940480 & -0.612410 & -1.614148 \\
\hline 0 & -1.993745 & -1.903478 & 0.199710 \\
\hline 0 & -1.379588 & -2.341117 & -2.113034 \\
\hline $\mathrm{H}$ & -0.939594 & -1.713293 & 1.283415 \\
\hline $\mathrm{H}$ & -0.431852 & -2.586896 & -1.975848 \\
\hline$S$ & 3.352894 & -0.067342 & 0.275553 \\
\hline 0 & 2.355719 & -0.320701 & -0.744415 \\
\hline 0 & 3.881681 & -1.225350 & 0.923700 \\
\hline
\end{tabular}




\begin{tabular}{|c|c|c|c|}
\hline 0 & 2.778118 & 0.924358 & 1.277620 \\
\hline 0 & 4.529190 & 0.703586 & -0.322917 \\
\hline $\mathrm{H}$ & 1.724111 & 0.953090 & 1.324418 \\
\hline $\mathrm{H}$ & 4.195631 & 1.597606 & -0.690164 \\
\hline 0 & -0.250494 & -1.545422 & 2.012420 \\
\hline $\mathrm{H}$ & -0.011714 & -0.549605 & 1.956087 \\
\hline $\mathrm{H}$ & 0.587864 & -2.115484 & 1.779348 \\
\hline 0 & 1.218776 & -2.704503 & -1.484749 \\
\hline $\mathrm{H}$ & 1.555269 & -1.799230 & -1.323166 \\
\hline $\mathrm{H}$ & 1.830897 & -3.112388 & -2.102697 \\
\hline 0 & 1.710470 & -2.936581 & 1.315822 \\
\hline $\mathrm{H}$ & 2.562736 & -2.467160 & 1.340945 \\
\hline $\mathrm{H}$ & 1.559924 & -3.126267 & 0.376420 \\
\hline 0 & -3.928359 & 2.575397 & 0.233047 \\
\hline $\mathrm{H}$ & -4.517129 & 1.234235 & 0.029994 \\
\hline $\mathrm{H}$ & -3.642318 & 3.011607 & -0.575744 \\
\hline 0 & 3.477494 & 2.875126 & -1.133996 \\
\hline $\mathrm{H}$ & 2.522401 & 2.748124 & -0.967575 \\
\hline $\mathrm{H}$ & 3.561809 & 3.122948 & -2.057218 \\
\hline $\mathrm{O}$ & -4.738789 & 0.220945 & 0.004112 \\
\hline $\mathrm{H}$ & -4.412202 & -0.147602 & 0.911400 \\
\hline $\mathrm{H}$ & -4.104926 & -0.171420 & -0.678331 \\
\hline 0 & -3.675183 & -0.607018 & 2.141316 \\
\hline $\mathrm{H}$ & -3.281749 & -1.436748 & 1.843572 \\
\hline $\mathrm{H}$ & -2.925850 & 0.016679 & 2.154810 \\
\hline
\end{tabular}

Table S64. Cartesian coordinate of $(\mathbf{S A})_{1} \mathbf{A}_{1}$.

\begin{tabular}{|c|c|c|c|}
\hline Atoms & $X$ & $Y$ & $Z$ \\
\hline$S$ & 0.598701 & -0.112566 & 0.085247 \\
\hline
\end{tabular}




\begin{tabular}{|l|l|l|l|}
\hline O & 1.008723 & 1.400067 & -0.155157 \\
O & -0.387978 & -0.388928 & -1.057140 \\
O & 1.757160 & -0.906230 & -0.109542 \\
O & -0.102665 & -0.107432 & 1.324252 \\
H & 1.743999 & 1.429704 & -0.779566 \\
H & -1.367686 & -0.203503 & -0.740444 \\
N & -2.731944 & 0.043273 & -0.051253 \\
H & -2.432364 & 0.084441 & 0.919179 \\
H & -3.405948 & -0.706646 & -0.145142 \\
H & -3.195540 & 0.914342 & -0.278505 \\
\hline
\end{tabular}

Table S65. Cartesian coordinate of $(\mathbf{S A})_{1} \mathbf{A}_{1} \mathbf{W}_{1}$.

\begin{tabular}{|c|c|c|c|}
\hline Atoms & X & Y & Z \\
\hline S & 0.029350 & 0.441626 & -0.025850 \\
O & -0.987181 & 1.276040 & 0.519351 \\
O & 1.093457 & 1.010728 & -0.790215 \\
O & -0.620434 & -0.658931 & -0.885415 \\
O & 0.648687 & -0.341693 & 1.172007 \\
H & -1.601887 & -0.808830 & -0.579182 \\
H & 1.545642 & -0.674998 & 0.910661 \\
N & -3.067373 & -0.785780 & -0.012617 \\
H & -3.037943 & 0.107606 & 0.471473 \\
H & -3.301177 & -1.503564 & 0.662167 \\
H & -3.807254 & -0.749736 & -0.702804 \\
O & 3.021078 & -0.817239 & 0.076781 \\
H & 3.185121 & -1.586031 & -0.472995 \\
H & 2.774652 & -0.101249 & -0.527467 \\
\hline
\end{tabular}

Table S66. Cartesian coordinate of $(\mathbf{S A})_{1} \mathbf{A}_{1} \mathbf{W}_{2}$. 


\begin{tabular}{|c|c|c|c|}
\hline Atoms & $\mathrm{X}$ & $\mathrm{Y}$ & $\mathrm{Z}$ \\
\hline $\mathrm{N}$ & 2.044363 & 0.277479 & 1.105483 \\
$\mathrm{H}$ & 1.103437 & -0.004915 & 1.467715 \\
$\mathrm{H}$ & 1.874615 & 1.084854 & 0.455997 \\
$\mathrm{H}$ & 2.382947 & -0.531876 & 0.549562 \\
$\mathrm{O}$ & 1.226768 & 2.238229 & -0.635138 \\
$\mathrm{H}$ & 0.268176 & 2.127766 & -0.476990 \\
$\mathrm{H}$ & 1.346110 & 1.934708 & -1.539087 \\
$\mathrm{O}$ & 2.205669 & -1.780625 & -0.692199 \\
$\mathrm{H}$ & 2.197148 & -2.731129 & -0.571648 \\
$\mathrm{H}$ & 1.286980 & -1.519607 & -0.909225 \\
$\mathrm{~S}$ & -1.023406 & -0.086326 & 0.061649 \\
$\mathrm{O}$ & -2.411290 & -0.850412 & -0.046405 \\
$\mathrm{O}$ & -0.536676 & -0.483103 & 1.369583 \\
$\mathrm{O}$ & -1.323639 & 1.324624 & -0.068977 \\
$\mathrm{O}$ & -0.181819 & -0.569818 & -1.022982 \\
$\mathrm{H}$ & -2.911451 & -0.478974 & -0.782679 \\
$\mathrm{H}$ & 2.683886 & 0.526872 & 1.850543 \\
\hline & & & \\
\hline & & & \\
\hline
\end{tabular}

Table S67. Cartesian coordinate of $(\mathbf{S A})_{1} \mathbf{A}_{1} \mathbf{W}_{3}$.

\begin{tabular}{|c|c|c|c|}
\hline Atoms & $\mathrm{X}$ & $\mathrm{Y}$ & $\mathrm{Z}$ \\
\hline $\mathrm{S}$ & 0.254957 & 1.059105 & 0.012842 \\
$\mathrm{O}$ & -0.604198 & 0.360729 & -0.927425 \\
$\mathrm{O}$ & -0.315307 & 2.545020 & -0.032655 \\
$\mathrm{O}$ & 1.639067 & 1.146017 & -0.385212 \\
$\mathrm{O}$ & 0.078929 & 0.594079 & 1.381843 \\
$\mathrm{H}$ & 0.316123 & 3.133823 & 0.397211 \\
$\mathrm{~N}$ & -1.259210 & -1.748181 & 1.089926 \\
$\mathrm{H}$ & -2.147853 & -1.463584 & 0.645664 \\
\hline
\end{tabular}




\begin{tabular}{|l|l|l|l|}
\hline $\mathrm{H}$ & -0.801133 & -0.876407 & 1.436853 \\
$\mathrm{H}$ & -1.412278 & -2.419494 & 1.832896 \\
$\mathrm{H}$ & -0.599145 & -2.122926 & 0.347685 \\
$\mathrm{O}$ & -3.163124 & -0.479953 & -0.485047 \\
$\mathrm{H}$ & -3.884454 & 0.141533 & -0.380806 \\
$\mathrm{H}$ & -2.390236 & 0.031596 & -0.784293 \\
$\mathrm{O}$ & 2.887350 & -1.299626 & 0.023415 \\
$\mathrm{H}$ & 3.238317 & -1.349901 & 0.913580 \\
$\mathrm{H}$ & 2.604368 & -0.375376 & -0.095691 \\
$\mathrm{O}$ & 0.496228 & -2.331211 & -0.853770 \\
$\mathrm{H}$ & 0.259616 & -1.555073 & -1.379264 \\
$\mathrm{H}$ & 1.400282 & -2.133042 & -0.537975 \\
\hline
\end{tabular}

Table S68. Cartesian coordinate of $(\mathbf{S A})_{1} \mathbf{A}_{1} \mathbf{W}_{4}$.

\begin{tabular}{|c|c|c|c|}
\hline Atoms & $\mathrm{X}$ & $\mathrm{Y}$ & $\mathrm{Z}$ \\
\hline $\mathrm{S}$ & 1.021668 & 0.729642 & 0.029082 \\
$\mathrm{O}$ & 2.424803 & 1.461399 & 0.172442 \\
$\mathrm{O}$ & 0.955900 & -0.079209 & 1.225014 \\
$\mathrm{O}$ & 0.023902 & 1.779591 & -0.041935 \\
$\mathrm{O}$ & 1.094544 & -0.049191 & -1.199728 \\
$\mathrm{H}$ & 2.562715 & 2.033371 & -0.592010 \\
$\mathrm{~N}$ & -1.141825 & -2.191564 & -0.020662 \\
$\mathrm{H}$ & -0.118645 & -2.398894 & 0.005706 \\
$\mathrm{H}$ & -1.672658 & -3.054754 & -0.058663 \\
$\mathrm{H}$ & -1.412225 & -1.624328 & 0.819362 \\
$\mathrm{H}$ & -1.352480 & -1.591250 & -0.856187 \\
$\mathrm{H}$ & 1.817074 & -2.094100 & 0.779248 \\
$\mathrm{O}$ & 1.622129 & -2.581027 & -0.030966 \\
$\mathrm{H}$ & 1.739237 & -1.895215 & -0.705764 \\
\hline
\end{tabular}




\begin{tabular}{|l|l|l|l|}
\hline$H$ & -3.167239 & 2.247406 & -0.028664 \\
$\mathrm{O}$ & -2.627055 & 1.455392 & -0.032744 \\
$\mathrm{H}$ & -1.691335 & 1.744539 & -0.017268 \\
$\mathrm{H}$ & -2.249781 & 0.285253 & 1.476482 \\
$\mathrm{O}$ & -1.762844 & -0.401254 & 1.956682 \\
$\mathrm{H}$ & -0.856950 & -0.063828 & 2.026378 \\
$\mathrm{H}$ & -2.133967 & 0.324638 & -1.549780 \\
$\mathrm{O}$ & -1.619694 & -0.363169 & -1.997963 \\
$\mathrm{H}$ & -0.711140 & -0.026423 & -2.025949 \\
\hline
\end{tabular}

Table S69. Cartesian coordinate of $(\mathbf{S A})_{1} \mathbf{A}_{1} \mathbf{W}_{5}$.

\begin{tabular}{|c|c|c|c|}
\hline Atoms & $x$ & $Y$ & $Z$ \\
\hline$S$ & 0.410341 & 1.108914 & -0.187593 \\
\hline 0 & -0.622145 & 0.414410 & 0.561478 \\
\hline 0 & -0.248661 & 2.547644 & -0.394768 \\
\hline 0 & 0.597709 & 0.557015 & -1.523415 \\
\hline 0 & 1.647599 & 1.312455 & 0.523557 \\
\hline $\mathrm{H}$ & 0.399496 & 3.140756 & -0.793452 \\
\hline$N$ & -0.707533 & -1.929660 & -1.149203 \\
\hline $\mathrm{H}$ & -0.454511 & -1.024529 & -1.566777 \\
\hline $\mathrm{H}$ & -0.719436 & -2.654196 & -1.858892 \\
\hline $\mathrm{H}$ & -1.647174 & -1.850805 & -0.709933 \\
\hline $\mathrm{H}$ & 0.062944 & -2.122752 & -0.431072 \\
\hline 0 & -3.317952 & 0.974567 & 0.450887 \\
\hline $\mathrm{H}$ & -2.351528 & 0.989555 & 0.556207 \\
\hline $\mathrm{H}$ & -3.536943 & 1.731076 & -0.095606 \\
\hline 0 & 1.096082 & -0.590674 & 2.656633 \\
\hline $\mathrm{H}$ & 1.631621 & 0.125990 & 2.293656 \\
\hline $\mathrm{H}$ & 0.209940 & -0.323226 & 2.385103 \\
\hline
\end{tabular}




\begin{tabular}{|l|l|l|l|}
\hline O & 1.368854 & -2.284242 & 0.462806 \\
$H$ & 1.312331 & -1.798372 & 1.308580 \\
$H$ & 2.103286 & -1.865445 & -0.023700 \\
O & 3.136935 & -0.726134 & -1.054758 \\
$H$ & 3.420663 & -0.080032 & -0.400541 \\
$H$ & 2.395357 & -0.272931 & -1.481146 \\
O & -3.317032 & -1.632853 & -0.252883 \\
$H$ & -3.446711 & -0.685183 & -0.037151 \\
$H$ & -3.723182 & -2.122409 & 0.464325 \\
\hline
\end{tabular}

Table S70. Cartesian coordinate of $(\mathbf{S A})_{2}$.

\begin{tabular}{|c|c|c|c|}
\hline Atoms & $X$ & $Y$ & $Z$ \\
\hline S & 2.035365 & 0.073179 & -0.114230 \\
O & 1.423634 & 0.890121 & 1.038482 \\
O & 1.067003 & -0.084348 & -1.156776 \\
O & 2.180490 & -1.369640 & 0.491648 \\
O & 3.310912 & 0.603152 & -0.396157 \\
H & 0.462447 & 0.657267 & 1.158023 \\
H & 2.959353 & -1.415425 & 1.062894 \\
S & -2.035370 & -0.073179 & 0.114229 \\
O & -2.180420 & 1.369656 & -0.491629 \\
O & -1.067011 & 0.084289 & 1.156788 \\
$O$ & -1.423664 & -0.890133 & -1.038488 \\
O & -3.310943 & -0.603099 & 0.396135 \\
H & -2.959260 & 1.415482 & -1.062902 \\
$\mathrm{H}$ & -0.462472 & -0.657301 & -1.158027 \\
\hline
\end{tabular}

Table S71. Cartesian coordinate of $(\mathbf{S A})_{2} \mathbf{W}_{1}$.

\begin{tabular}{|c|c|c|c|}
\hline Atoms & $\mathrm{X}$ & $\mathrm{Y}$ & $\mathrm{Z}$ \\
\hline
\end{tabular}




\begin{tabular}{|l|l|l|l|}
\hline S & -1.460321 & -0.680851 & -0.062989 \\
O & -1.136151 & -0.665008 & 1.445079 \\
O & -0.472080 & -1.435517 & -0.763813 \\
O & -1.230284 & 0.803924 & -0.498788 \\
O & -2.835220 & -0.998806 & -0.195667 \\
H & -0.178088 & -0.423888 & 1.558214 \\
H & -2.069308 & 1.338574 & -0.309121 \\
S & 2.227427 & 0.247502 & 0.099560 \\
O & 1.562981 & 1.233111 & -0.910716 \\
O & 3.561877 & 0.645549 & 0.279774 \\
O & 2.234237 & -1.106578 & -0.654776 \\
O & 1.349547 & 0.136318 & 1.234572 \\
H & 0.590132 & 1.204536 & -0.844447 \\
H & 1.315188 & -1.433923 & -0.760740 \\
O & -3.552470 & 1.774271 & -0.054426 \\
H & -3.803513 & 2.238350 & 0.748015 \\
H & -3.987598 & 0.911817 & -0.026967 \\
\hline
\end{tabular}

Table S72. Cartesian coordinate of $(\mathbf{S A})_{2} \mathbf{W}_{2}$.

\begin{tabular}{|c|c|c|c|}
\hline Atoms & $X$ & $Y$ & $Z$ \\
\hline S & 2.168579 & -0.112973 & -0.007448 \\
O & 1.940366 & -1.621859 & 0.214116 \\
O & 1.514025 & 0.180884 & -1.382703 \\
O & 1.416872 & 0.595317 & 1.002887 \\
O & 3.550011 & 0.121570 & -0.119939 \\
H & 0.973769 & -1.812646 & 0.341597 \\
H & 0.524216 & 0.111239 & -1.331587 \\
S & -1.603000 & -0.780715 & 0.014263 \\
O & -2.877166 & -1.434309 & -0.637785 \\
\hline
\end{tabular}




\begin{tabular}{|l|l|l|l|}
\hline O & -0.698083 & -1.853290 & 0.346916 \\
$\mathrm{O}$ & -1.110572 & 0.095335 & -1.044099 \\
$\mathrm{O}$ & -2.073729 & -0.033428 & 1.172412 \\
$\mathrm{H}$ & -3.235365 & -2.115325 & -0.053316 \\
$\mathrm{O}$ & -0.654928 & 2.027842 & 1.398255 \\
$\mathrm{H}$ & -0.884292 & 2.503744 & 0.529457 \\
$\mathrm{H}$ & -1.298818 & 1.225178 & 1.432128 \\
$\mathrm{H}$ & 0.242212 & 1.592962 & 1.272083 \\
$\mathrm{H}$ & -1.441827 & 1.961393 & -1.277186 \\
$\mathrm{O}$ & -1.385668 & 2.850406 & -0.889942 \\
$\mathrm{H}$ & -0.898160 & 3.404724 & -1.503149 \\
\hline
\end{tabular}

Table S73. Cartesian coordinate of $(\mathbf{S A})_{2} \mathbf{W}_{3}$.

\begin{tabular}{|c|c|c|c|}
\hline Atoms & $\mathrm{X}$ & $\mathrm{Y}$ & $\mathrm{Z}$ \\
\hline $\mathrm{S}$ & 1.658037 & -0.904255 & -0.000025 \\
$\mathrm{O}$ & 0.838390 & -0.888392 & -1.207484 \\
$\mathrm{O}$ & 0.838468 & -0.888418 & 1.207495 \\
$\mathrm{O}$ & 2.442101 & -2.267695 & -0.000040 \\
$\mathrm{O}$ & 2.714768 & 0.073775 & -0.000068 \\
$\mathrm{H}$ & 1.222953 & 2.380545 & 0.828867 \\
$\mathrm{~S}$ & -2.264842 & -0.178316 & 0.000009 \\
$\mathrm{O}$ & -1.775428 & -0.991869 & -1.221765 \\
$\mathrm{O}$ & -1.775326 & -0.991974 & 1.221667 \\
$\mathrm{O}$ & -1.550522 & 1.082079 & 0.000035 \\
$\mathrm{O}$ & -3.670289 & -0.170368 & 0.000065 \\
$\mathrm{H}$ & -0.777973 & -1.005838 & 1.261875 \\
$\mathrm{H}$ & -0.778083 & -1.005806 & -1.261979 \\
$\mathrm{O}$ & 0.322950 & 1.907600 & -1.904016 \\
$\mathrm{H}$ & -0.523423 & 1.732554 & -1.458032 \\
\hline & & & \\
\hline
\end{tabular}




\begin{tabular}{|l|l|l|l|}
\hline $\mathrm{H}$ & 0.641774 & 1.027273 & -2.148208 \\
$\mathrm{O}$ & 1.836727 & 2.523434 & -0.000012 \\
$\mathrm{H}$ & 2.394823 & 1.699606 & -0.000033 \\
$\mathrm{H}$ & 1.222896 & 2.380540 & -0.828872 \\
$\mathrm{H}$ & 1.828535 & -3.013516 & 0.000466 \\
$\mathrm{O}$ & 0.323018 & 1.907579 & 1.904090 \\
$\mathrm{H}$ & -0.523369 & 1.732502 & 1.458149 \\
$\mathrm{H}$ & 0.641872 & 1.027265 & 2.148286 \\
\hline
\end{tabular}

Table S74. Cartesian coordinate of $(\mathbf{S A})_{2} \mathbf{W}_{4}$.

\begin{tabular}{|c|c|c|c|}
\hline Atoms & $\mathrm{X}$ & $\mathrm{Y}$ & $\mathrm{Z}$ \\
\hline $\mathrm{S}$ & -1.856780 & 0.565211 & -0.179448 \\
$\mathrm{O}$ & -1.089496 & 0.348264 & 1.093117 \\
$\mathrm{O}$ & -0.651010 & -2.155711 & 1.247215 \\
$\mathrm{H}$ & -0.839520 & -1.161273 & 1.349591 \\
$\mathrm{O}$ & -3.174895 & 0.006770 & -0.093148 \\
$\mathrm{O}$ & -1.130336 & -0.332177 & -1.234322 \\
$\mathrm{O}$ & -2.693953 & -2.715575 & -0.126691 \\
$\mathrm{H}$ & -1.430980 & -2.498295 & 0.664192 \\
$\mathrm{O}$ & 0.842681 & 1.943739 & 1.520908 \\
$\mathrm{H}$ & 1.599854 & 1.290926 & 1.351121 \\
$\mathrm{~S}$ & 2.136868 & -0.625984 & -0.183789 \\
$\mathrm{O}$ & 1.395848 & 0.229068 & -1.096981 \\
$\mathrm{O}$ & 1.536258 & -1.944854 & -0.045200 \\
$\mathrm{O}$ & 2.443307 & 0.020849 & 1.074947 \\
$\mathrm{H}$ & -0.157231 & -0.104742 & -1.284024 \\
$\mathrm{O}$ & -1.754479 & 1.941623 & -0.582383 \\
$\mathrm{H}$ & -2.517389 & -2.938576 & -1.044903 \\
$\mathrm{H}$ & -3.089280 & -1.820655 & -0.154284 \\
\hline & & & \\
\hline
\end{tabular}




\begin{tabular}{|l|l|l|l|}
\hline $\mathrm{H}$ & 0.224191 & -2.178546 & 0.732974 \\
$\mathrm{H}$ & 0.007917 & 1.367084 & 1.423831 \\
$\mathrm{H}$ & 0.784161 & 2.652394 & 0.777463 \\
$\mathrm{O}$ & 3.557789 & -0.863657 & -0.818833 \\
$\mathrm{H}$ & 3.469894 & -1.346774 & -1.650175 \\
$\mathrm{H}$ & 0.189432 & 4.453139 & -0.187537 \\
$\mathrm{O}$ & 0.426665 & 3.542253 & -0.375567 \\
$\mathrm{H}$ & -0.389494 & 3.092955 & -0.670967 \\
\hline
\end{tabular}

Table S75. Cartesian coordinate of $(\mathbf{S A})_{2} \mathbf{W}_{5}$.

\begin{tabular}{|c|c|c|c|}
\hline Atoms & $\mathrm{X}$ & $\mathrm{Y}$ & $\mathrm{Z}$ \\
\hline $\mathrm{S}$ & 2.536717 & 0.064430 & -0.144640 \\
$\mathrm{O}$ & 1.752639 & -0.479030 & 0.944371 \\
$\mathrm{O}$ & 4.025402 & -0.184773 & 0.318889 \\
$\mathrm{O}$ & 2.403568 & 1.498181 & -0.318429 \\
$\mathrm{O}$ & 2.373718 & -0.665927 & -1.393042 \\
$\mathrm{H}$ & 4.636287 & 0.164279 & -0.342804 \\
$\mathrm{~S}$ & -2.363018 & -0.285407 & 0.029788 \\
$\mathrm{O}$ & -1.551791 & -0.284529 & -1.183643 \\
$\mathrm{O}$ & -3.674454 & -1.033113 & -0.450936 \\
$\mathrm{O}$ & -2.751258 & 1.038808 & 0.454585 \\
$\mathrm{O}$ & -1.799157 & -1.076146 & 1.106822 \\
$\mathrm{H}$ & -4.315492 & -1.056725 & 0.270701 \\
$\mathrm{O}$ & 0.262195 & -2.073250 & -1.476572 \\
$\mathrm{H}$ & 1.123359 & -1.535490 & -1.533376 \\
$\mathrm{H}$ & 0.276135 & -2.518797 & -0.530250 \\
$\mathrm{H}$ & -0.493332 & -1.403487 & -1.443762 \\
$\mathrm{O}$ & 0.250077 & 2.712194 & 0.388380 \\
$\mathrm{H}$ & 1.112805 & 2.319203 & 0.057005 \\
\hline & & & \\
\hline
\end{tabular}




\begin{tabular}{|l|r|r|r|}
\hline $\mathrm{H}$ & -0.039397 & 2.095425 & 1.175056 \\
$\mathrm{H}$ & -0.449489 & 2.701732 & -0.366251 \\
$\mathrm{O}$ & -0.178456 & 1.091704 & 2.242098 \\
$\mathrm{H}$ & -1.001142 & 0.590814 & 2.158559 \\
$\mathrm{H}$ & 0.523319 & 0.457527 & 2.016926 \\
$\mathrm{O}$ & -1.524406 & 2.634803 & -1.414624 \\
$\mathrm{H}$ & -2.259128 & 2.325400 & -0.857404 \\
$\mathrm{H}$ & -1.340823 & 1.860654 & -1.961264 \\
$\mathrm{O}$ & 0.285602 & -2.892285 & 0.884427 \\
$\mathrm{H}$ & -0.531905 & -2.449338 & 1.171112 \\
$\mathrm{H}$ & 0.990189 & -2.308650 & 1.204776 \\
\hline
\end{tabular}

Table S76. Cartesian coordinate of $(\mathbf{S A})_{2} \mathbf{W}_{6}$.

\begin{tabular}{|c|c|c|c|}
\hline Atoms & $\mathrm{X}$ & $\mathrm{Y}$ & $\mathrm{Z}$ \\
\hline $\mathrm{S}$ & 2.388277 & -0.052619 & -0.085444 \\
$\mathrm{O}$ & 1.703805 & -0.687203 & 1.017423 \\
$\mathrm{O}$ & 3.912168 & -0.222106 & 0.292051 \\
$\mathrm{O}$ & 2.149133 & 1.375526 & -0.203850 \\
$\mathrm{O}$ & 2.203069 & -0.730280 & -1.360713 \\
$\mathrm{H}$ & 4.466298 & 0.186319 & -0.385422 \\
$\mathrm{~S}$ & -2.268250 & -0.437175 & -0.033414 \\
$\mathrm{O}$ & -1.489260 & -0.584267 & -1.260093 \\
$\mathrm{O}$ & -3.668069 & -1.032379 & -0.470677 \\
$\mathrm{O}$ & -2.484481 & 0.930074 & 0.363337 \\
$\mathrm{O}$ & -1.782157 & -1.265632 & 1.054985 \\
$\mathrm{H}$ & -4.297093 & -0.949952 & 0.257157 \\
$\mathrm{O}$ & 0.288934 & -2.413613 & -1.430647 \\
$\mathrm{H}$ & 1.103099 & -1.813692 & -1.483694 \\
$\mathrm{H}$ & 0.295839 & -2.816106 & -0.462177 \\
\hline & & & \\
\hline & & & \\
\hline
\end{tabular}




\begin{tabular}{|l|l|l|l|}
\hline $\mathrm{H}$ & -0.491639 & -1.772193 & -1.443715 \\
$\mathrm{O}$ & 0.336158 & 2.692702 & 1.235845 \\
$\mathrm{H}$ & 1.091062 & 2.305939 & 0.729257 \\
$\mathrm{H}$ & 0.001695 & 1.920088 & 1.825241 \\
$\mathrm{H}$ & -0.418791 & 2.992542 & 0.514302 \\
$\mathrm{O}$ & -0.225593 & 0.638031 & 2.592955 \\
$\mathrm{H}$ & -1.025297 & 0.179647 & 2.299102 \\
$\mathrm{H}$ & 0.496682 & 0.084739 & 2.254862 \\
$\mathrm{O}$ & -1.314561 & 3.281850 & -0.472987 \\
$\mathrm{H}$ & -2.044288 & 2.673354 & -0.275767 \\
$\mathrm{H}$ & -0.876284 & 2.861806 & -1.252582 \\
$\mathrm{O}$ & 0.270826 & -3.123941 & 0.960341 \\
$\mathrm{H}$ & -0.548295 & -2.659113 & 1.206367 \\
$\mathrm{H}$ & 0.972768 & -2.538095 & 1.281248 \\
$\mathrm{O}$ & -0.025410 & 1.728955 & -2.221613 \\
$\mathrm{H}$ & -0.505097 & 0.904999 & -2.057117 \\
$\mathrm{H}$ & 0.862413 & 1.574680 & -1.876178 \\
\hline & & & \\
\hline
\end{tabular}

Table S77. Cartesian coordinate of $(\mathbf{S A})_{2} \mathbf{W}_{7}$.

\begin{tabular}{|c|c|c|c|}
\hline Atoms & $X$ & $Y$ & $Z$ \\
\hline S & 2.624290 & -0.104566 & -0.155364 \\
$O$ & 1.830762 & -0.303430 & -1.345772 \\
O & 4.087096 & -0.452718 & -0.632579 \\
O & 2.307579 & -1.038104 & 0.920956 \\
O & 2.662739 & 1.263628 & 0.325012 \\
H & 4.712441 & -0.304857 & 0.088436 \\
S & -2.449013 & -0.052676 & -0.454121 \\
O & -1.624871 & 0.837081 & 0.343279 \\
$O$ & -3.853412 & 0.683238 & -0.432409 \\
\hline
\end{tabular}




\begin{tabular}{|c|c|c|c|}
\hline 0 & -2.645053 & -1.364324 & 0.116808 \\
\hline $\mathrm{O}$ & -2.032020 & -0.109189 & -1.847561 \\
\hline $\mathrm{H}$ & -4.497299 & 0.165191 & -0.931514 \\
\hline 0 & 0.715761 & 2.926322 & -0.121546 \\
\hline $\mathrm{H}$ & 1.506231 & 2.365725 & 0.113620 \\
\hline $\mathrm{H}$ & 0.297954 & 2.453583 & -0.969502 \\
\hline $\mathrm{H}$ & 0.083742 & 2.960432 & 0.684184 \\
\hline $\mathrm{O}$ & 0.406287 & -2.818690 & 0.602603 \\
\hline $\mathrm{H}$ & 1.190737 & -2.219732 & 0.730950 \\
\hline $\mathrm{H}$ & 0.059283 & -2.639914 & -0.359909 \\
\hline $\mathrm{H}$ & -0.309796 & -2.577659 & 1.335169 \\
\hline 0 & -0.165552 & -2.264179 & -1.770742 \\
\hline $\mathrm{H}$ & -0.965272 & -1.732208 & -1.912059 \\
\hline $\mathrm{H}$ & 0.570569 & -1.653656 & -1.928057 \\
\hline $\mathrm{O}$ & -1.255734 & -2.140008 & 2.326160 \\
\hline $\mathrm{H}$ & -1.985347 & -1.846578 & 1.750340 \\
\hline $\mathrm{H}$ & -0.871575 & -1.324138 & 2.688735 \\
\hline 0 & 0.032136 & 1.718980 & -2.167675 \\
\hline $\mathrm{H}$ & -0.783372 & 1.184951 & -2.120770 \\
\hline $\mathrm{H}$ & 0.747540 & 1.064021 & -2.179213 \\
\hline $\mathrm{O}$ & 0.160058 & 0.304444 & 2.379040 \\
\hline $\mathrm{H}$ & -0.304713 & 0.376084 & 1.527691 \\
\hline $\mathrm{H}$ & 1.058484 & 0.024001 & 2.160072 \\
\hline 0 & -0.794821 & 2.939868 & 1.928861 \\
\hline $\mathrm{H}$ & -1.579553 & 2.490656 & 1.585923 \\
\hline $\mathrm{H}$ & -0.382129 & 2.266620 & 2.492183 \\
\hline
\end{tabular}

Table S78. Cartesian coordinate of $(\mathbf{S A})_{2} \mathbf{A}_{1}$.

\begin{tabular}{|c|c|c|c|}
\hline Atoms & $\mathrm{X}$ & $\mathrm{Y}$ & $\mathrm{Z}$ \\
\hline
\end{tabular}




\begin{tabular}{|l|l|l|l|}
\hline S & 1.780537 & -0.350791 & -0.049136 \\
O & 2.395462 & 0.755681 & -0.764877 \\
O & 1.080155 & 0.142334 & 1.140082 \\
O & 2.963043 & -1.265437 & 0.453993 \\
H & 0.987268 & -1.244923 & -0.848949 \\
H & 1.426284 & 2.076968 & -0.427545 \\
S & 3.664760 & -0.718983 & 0.827862 \\
O & -2.076443 & -0.119175 & -0.011823 \\
O & -1.445033 & -0.409398 & 1.369034 \\
O & -3.477924 & -0.145654 & 0.115487 \\
O & -1.644788 & -1.308567 & -0.893008 \\
H & -1.454162 & 1.076500 & -0.540365 \\
$\mathrm{H}$ & -0.452948 & -0.256205 & 1.349764 \\
$\mathrm{~N}$ & -0.652732 & -1.340057 & -0.972091 \\
$\mathrm{H}$ & 0.631706 & 2.651699 & -0.045424 \\
$\mathrm{H}$ & -0.240934 & 2.080913 & -0.198980 \\
$\mathrm{H}$ & 0.560393 & 3.559485 & -0.492964 \\
\hline & 0.775564 & 2.751155 & 0.956084 \\
\hline
\end{tabular}

Table S79. Cartesian coordinate of $(\mathbf{S A})_{2} \mathbf{A}_{1} \mathbf{W}_{1}$.

\begin{tabular}{|c|c|c|c|}
\hline Atoms & $\mathrm{X}$ & $\mathrm{Y}$ & $\mathrm{Z}$ \\
\hline $\mathrm{S}$ & -2.230852 & 0.044075 & 0.037008 \\
$\mathrm{O}$ & -2.132880 & -1.454101 & -0.328047 \\
$\mathrm{O}$ & -1.460518 & 0.791013 & -0.926694 \\
$\mathrm{O}$ & -1.509323 & 0.151411 & 1.405004 \\
$\mathrm{O}$ & -3.589610 & 0.362270 & 0.224289 \\
$\mathrm{H}$ & -1.180090 & -1.718773 & -0.419229 \\
$\mathrm{H}$ & -0.520870 & 0.115357 & 1.285288 \\
$\mathrm{~S}$ & 1.503940 & -0.919523 & -0.063789 \\
\hline
\end{tabular}




\begin{tabular}{|l|l|l|l|}
\hline O & 0.477492 & -1.895383 & -0.348176 \\
$\mathrm{O}$ & 2.629944 & -1.669478 & 0.753814 \\
$\mathrm{O}$ & 1.070545 & 0.112166 & 0.880012 \\
$\mathrm{O}$ & 2.134939 & -0.362437 & -1.239516 \\
$\mathrm{H}$ & 2.966321 & -2.411618 & 0.235801 \\
$\mathrm{~N}$ & 0.890747 & 2.083506 & -1.405521 \\
$\mathrm{H}$ & -0.065411 & 1.722676 & -1.216725 \\
$\mathrm{H}$ & 1.243974 & 2.570076 & -0.560303 \\
$\mathrm{H}$ & 1.486782 & 1.244484 & -1.553070 \\
$\mathrm{O}$ & 1.995546 & 2.730985 & 1.032331 \\
$\mathrm{H}$ & 1.843375 & 1.808214 & 1.289401 \\
$\mathrm{H}$ & 1.796950 & 3.270161 & 1.799607 \\
$\mathrm{H}$ & 0.895236 & 2.690484 & -2.217760 \\
\hline
\end{tabular}

Table S80. Cartesian coordinate of $(\mathbf{S A})_{2} \mathbf{A}_{1} \mathbf{W}_{2}$.

\begin{tabular}{|c|c|c|c|}
\hline Atoms & $X$ & $Y$ & $Z$ \\
\hline S & -2.439383 & -0.166384 & -0.148815 \\
$O$ & -2.303218 & 1.113452 & -0.796023 \\
O & -3.981338 & -0.353243 & 0.157645 \\
O & -2.118886 & -1.325613 & -0.950125 \\
H & -4.358607 & 0.494614 & 0.421208 \\
S & 2.438468 & -0.153992 & -0.135130 \\
$O$ & 2.213447 & 1.057162 & -0.881832 \\
$O$ & 1.749336 & -0.198447 & 1.153183 \\
$O$ & 3.962905 & -0.148779 & 0.296145 \\
$O$ & 2.225278 & -1.371211 & -0.897346 \\
H & 0.897224 & -2.168100 & -0.281492 \\
$O$ & -1.755410 & -0.211648 & 1.151297 \\
$O$ & -0.017507 & 1.611482 & 1.538094 \\
\hline
\end{tabular}




\begin{tabular}{|l|r|r|r|}
\hline $\mathrm{N}$ & 0.024068 & -2.454620 & 0.227802 \\
$\mathrm{O}$ & -0.015328 & 2.688398 & -0.689642 \\
$\mathrm{H}$ & 0.024545 & -1.955005 & 1.117965 \\
$\mathrm{H}$ & -0.825126 & -2.130545 & -0.300859 \\
$\mathrm{H}$ & 4.512110 & 0.004725 & -0.482602 \\
$\mathrm{H}$ & -0.803200 & 2.261794 & -1.065128 \\
$\mathrm{H}$ & 0.758501 & 2.238994 & -1.069754 \\
$\mathrm{H}$ & -0.795805 & 0.966091 & 1.462921 \\
$\mathrm{H}$ & -0.017026 & 2.162366 & 0.630018 \\
$\mathrm{H}$ & 0.780805 & 0.997433 & 1.481615 \\
$\mathrm{H}$ & -0.001487 & -3.456429 & 0.383454 \\
\hline
\end{tabular}

Table S81. Cartesian coordinate of $(\mathbf{S A})_{2} \mathbf{A}_{1} \mathbf{W}_{3}$.

\begin{tabular}{|c|c|c|c|}
\hline Atoms & $\mathrm{X}$ & $\mathrm{Y}$ & $\mathrm{Z}$ \\
\hline $\mathrm{S}$ & 2.111424 & -0.795585 & -0.162589 \\
$\mathrm{O}$ & 3.576930 & -1.030117 & -0.703513 \\
$\mathrm{O}$ & 1.423566 & -2.063947 & -0.271836 \\
$\mathrm{O}$ & 2.334450 & -0.342506 & 1.195481 \\
$\mathrm{O}$ & 1.512193 & 0.233219 & -1.009120 \\
$\mathrm{H}$ & 3.538353 & -1.479843 & -1.556887 \\
$\mathrm{~S}$ & -1.782808 & 0.841269 & -0.124000 \\
$\mathrm{O}$ & -1.137087 & -0.201916 & -1.116391 \\
$\mathrm{O}$ & -3.174308 & 0.492807 & -0.134006 \\
$\mathrm{O}$ & -1.147995 & 0.537831 & 1.190746 \\
$\mathrm{O}$ & -1.436976 & 2.165144 & -0.566668 \\
$\mathrm{H}$ & -0.167682 & -0.035574 & -1.206409 \\
$\mathrm{~N}$ & -1.028694 & -2.233870 & 1.010858 \\
$\mathrm{H}$ & -1.897138 & -2.415723 & 0.459925 \\
$\mathrm{H}$ & -0.963961 & -2.860319 & 1.806074 \\
\hline
\end{tabular}




\begin{tabular}{|l|l|l|l|}
\hline $\mathrm{H}$ & -0.174724 & -2.310606 & 0.427070 \\
$\mathrm{H}$ & -1.075592 & -1.244822 & 1.324310 \\
$\mathrm{O}$ & 0.991070 & 1.804851 & 1.657194 \\
$\mathrm{H}$ & 1.623568 & 1.027434 & 1.566538 \\
$\mathrm{H}$ & 1.121455 & 2.393767 & 0.803377 \\
$\mathrm{H}$ & 0.070674 & 1.368818 & 1.541836 \\
$\mathrm{H}$ & -3.477670 & -1.276993 & -0.430696 \\
$\mathrm{O}$ & -3.390245 & -2.248016 & -0.383006 \\
$\mathrm{H}$ & -4.260398 & -2.588009 & -0.169890 \\
$\mathrm{H}$ & 1.578234 & 2.326608 & -1.042924 \\
$\mathrm{O}$ & 1.173619 & 3.031209 & -0.517968 \\
$\mathrm{H}$ & 0.226131 & 2.972944 & -0.740205 \\
\hline
\end{tabular}

Table S82. Cartesian coordinate of $(\mathbf{S A})_{2} \mathbf{A}_{1} \mathbf{W}_{4}$.

\begin{tabular}{|c|c|c|c|}
\hline Atoms & $\mathrm{X}$ & $\mathrm{Y}$ & $\mathrm{Z}$ \\
\hline $\mathrm{S}$ & -1.861767 & 0.740978 & -0.022891 \\
$\mathrm{O}$ & -0.981151 & 0.498306 & 1.128133 \\
$\mathrm{O}$ & -3.033826 & -0.115978 & -0.006037 \\
$\mathrm{O}$ & -1.047503 & 0.235867 & -1.279426 \\
$\mathrm{O}$ & -2.133015 & 2.139164 & -0.218836 \\
$\mathrm{H}$ & -0.066415 & 0.384458 & -1.145889 \\
$\mathrm{O}$ & -0.409036 & -2.058327 & 2.002146 \\
$\mathrm{H}$ & 0.427782 & -2.207378 & 1.537107 \\
$\mathrm{H}$ & -1.518537 & -2.411691 & 1.082293 \\
$\mathrm{H}$ & -0.514729 & -1.091497 & 1.955617 \\
$\mathrm{O}$ & -2.233493 & -2.511813 & 0.333750 \\
$\mathrm{H}$ & -1.685681 & -2.646588 & -0.518988 \\
$\mathrm{H}$ & -2.667725 & -1.592435 & 0.230257 \\
$\mathrm{O}$ & 0.279527 & 3.333705 & -1.032289 \\
\hline
\end{tabular}




\begin{tabular}{|l|l|l|l|}
\hline $\mathrm{H}$ & 0.698681 & 2.723828 & -1.647299 \\
$\mathrm{H}$ & 0.809390 & 2.870691 & 0.502496 \\
$\mathrm{H}$ & -0.654755 & 3.065039 & -0.986613 \\
$\mathrm{~S}$ & 2.126457 & -0.429454 & -0.069624 \\
$\mathrm{O}$ & 1.493676 & 0.569581 & -0.934273 \\
$\mathrm{O}$ & 1.455944 & -1.716377 & -0.145334 \\
$\mathrm{O}$ & 2.369366 & 0.048650 & 1.264734 \\
$\mathrm{O}$ & 3.581004 & -0.647374 & -0.654054 \\
$\mathrm{H}$ & 3.528971 & -1.088708 & -1.510597 \\
$\mathrm{~N}$ & 0.910172 & 2.425321 & 1.450200 \\
$\mathrm{H}$ & 0.064772 & 1.832610 & 1.567997 \\
$\mathrm{H}$ & 1.700693 & 1.762643 & 1.454490 \\
$\mathrm{H}$ & -0.977569 & -1.780130 & -2.141735 \\
$\mathrm{O}$ & -0.702152 & -2.594312 & -1.703181 \\
$\mathrm{H}$ & 0.139566 & -2.350980 & -1.276538 \\
$\mathrm{H}$ & 0.994580 & 3.119800 & 2.183595 \\
\hline
\end{tabular}

Table S83. Cartesian coordinate of $(\mathbf{S A})_{2} \mathbf{A}_{1} \mathbf{W}_{5}$.

\begin{tabular}{|c|c|c|c|}
\hline Atoms & $\mathrm{X}$ & $\mathrm{Y}$ & $\mathrm{Z}$ \\
\hline $\mathrm{S}$ & 1.774147 & 0.949562 & -0.495563 \\
$\mathrm{O}$ & 3.141241 & 0.696707 & -0.118981 \\
$\mathrm{O}$ & 1.428582 & -0.275287 & -1.434493 \\
$\mathrm{O}$ & 0.828721 & 0.866380 & 0.625362 \\
$\mathrm{H}$ & 0.493090 & -0.199582 & -1.730927 \\
$\mathrm{~S}$ & -1.918521 & -0.766108 & -0.856314 \\
$\mathrm{O}$ & -1.217290 & 0.059056 & -1.826592 \\
$\mathrm{O}$ & -3.045476 & -1.441979 & -1.743219 \\
$\mathrm{O}$ & -2.599857 & 0.023771 & 0.152484 \\
$\mathrm{H}$ & -3.522158 & -2.094602 & -1.216294 \\
\hline
\end{tabular}




\begin{tabular}{|c|c|c|c|}
\hline $\mathrm{N}$ & -1.091681 & 2.694303 & -0.704135 \\
\hline $\mathrm{H}$ & -1.468731 & 1.836303 & -1.125360 \\
\hline 0 & 3.496002 & -1.971344 & -0.066112 \\
\hline $\mathrm{H}$ & 2.110756 & -2.326822 & 0.361888 \\
\hline $\mathrm{H}$ & 3.682713 & -2.234898 & -0.970607 \\
\hline $\mathrm{H}$ & 3.517993 & -0.987831 & -0.072516 \\
\hline $\mathrm{O}$ & 0.745960 & -0.961111 & 2.579862 \\
\hline $\mathrm{H}$ & -0.197747 & -0.872065 & 2.802178 \\
\hline $\mathrm{H}$ & 0.914907 & -0.183278 & 2.007542 \\
\hline 0 & 1.179240 & -2.541252 & 0.750195 \\
\hline $\mathrm{H}$ & 0.994115 & -1.936882 & 1.610866 \\
\hline 0 & -1.898918 & -0.107273 & 2.748681 \\
\hline $\mathrm{H}$ & -2.624619 & -0.401789 & 3.301981 \\
\hline $\mathrm{H}$ & -2.204375 & -0.175446 & 1.819824 \\
\hline $\mathrm{O}$ & -0.954786 & 2.505288 & 2.025717 \\
\hline $\mathrm{H}$ & -0.077665 & 2.127531 & 1.870907 \\
\hline $\mathrm{H}$ & -1.440887 & 1.775067 & 2.436145 \\
\hline 0 & 1.545643 & 2.159278 & -1.260077 \\
\hline $\mathrm{H}$ & -0.073831 & 2.697282 & -0.940134 \\
\hline $\mathrm{O}$ & -1.131253 & -1.847393 & -0.306368 \\
\hline $\mathrm{H}$ & 0.460537 & -2.285653 & 0.126117 \\
\hline $\mathrm{H}$ & -1.559899 & 3.514284 & -1.073139 \\
\hline $\mathrm{H}$ & -1.194897 & 2.634280 & 0.338841 \\
\hline
\end{tabular}

Table S84. AIM topological parameters for the stable clusters obtained at the M06-2X/6-311++G(3df,3pd) level (in a.u.).

\begin{tabular}{|l|l|c|c|c|}
\hline Clusters & Bonds & $\mathrm{r}$ & $\rho$ & $\nabla^{2} \rho$ \\
\hline$(\mathbf{G A})_{1} \cdot \mathbf{A}_{1}$ & $\mathrm{C}=\mathrm{O} \ldots \mathrm{H}-\mathrm{N}$ & 2.460 & 0.0115 & 0.0392 \\
\hline
\end{tabular}




\begin{tabular}{|c|c|c|c|c|}
\hline & C-O-H...N-H & 1.683 & 0.0548 & 0.1281 \\
\hline \multirow[t]{2}{*}{$(\mathbf{G W})_{1} \cdot \mathbf{A}_{1}$} & $\mathrm{C}=\mathrm{O} \ldots \mathrm{H}-\mathrm{N}$ & 2.428 & 0.0120 & 0.0403 \\
\hline & C-O-H...N-H & 1.695 & 0.0531 & 0.1277 \\
\hline \multirow[t]{2}{*}{$(\mathbf{G A})_{1} \cdot \mathbf{A}_{1} \cdot \mathbf{W}_{1}$} & $\mathrm{C}=\mathrm{O} \ldots \mathrm{H}-\mathrm{O}-\mathrm{H}$ & 1.840 & 0.0293 & 0.1139 \\
\hline & C-O-H...N-H & 1.582 & 0.0712 & 0.1084 \\
\hline \multirow[t]{2}{*}{$(\mathbf{G W})_{1} \cdot \mathbf{A}_{1} \cdot \mathbf{W}_{1}$} & $\mathrm{C}=\mathrm{O} \ldots \mathrm{H}-\mathrm{O}-\mathrm{H}$ & 1.831 & 0.0298 & 0.1164 \\
\hline & C-O-H...N-H & 1.598 & 0.0684 & 0.1134 \\
\hline \multirow[t]{2}{*}{$(\mathbf{G A})_{1} \cdot(\mathbf{S A})_{1}$} & $\mathrm{C}=\mathrm{O} \ldots \mathrm{H}-\mathrm{O}-\mathrm{S}$ & 1.571 & 0.0582 & 0.1761 \\
\hline & $\mathrm{C}-\mathrm{O}-\mathrm{H} \ldots \mathrm{O}=\mathrm{S}$ & 1.668 & 0.0432 & 0.1619 \\
\hline \multirow[t]{3}{*}{$(\mathbf{G W})_{1} \cdot(\mathbf{S A})_{1}$} & $\mathrm{C}=\mathrm{O} \ldots \mathrm{H}-\mathrm{O}-\mathrm{S}$ & 1.849 & 0.0274 & 0.1102 \\
\hline & $\mathrm{O}-\mathrm{H} \ldots \mathrm{O}=\mathrm{S}$ & 1.839 & 0.0289 & 0.1127 \\
\hline & $\mathrm{H}-\mathrm{O} \ldots \mathrm{H}-\mathrm{O}-\mathrm{S}$ & 1.731 & 0.0398 & 0.1400 \\
\hline \multirow[t]{2}{*}{$(\mathbf{G A})_{1} \cdot(\mathbf{S A})_{1} \cdot \mathbf{W}_{1}$} & $\mathrm{C}=\mathrm{O} \ldots \mathrm{H}-\mathrm{O}-\mathrm{S}$ & 1.592 & 0.0552 & 0.1744 \\
\hline & $\mathrm{C}-\mathrm{O}-\mathrm{H} \ldots \mathrm{O}=\mathrm{S}$ & 1.654 & 0.0451 & 0.1652 \\
\hline \multirow[t]{3}{*}{$(\mathbf{G W})_{1} \cdot(\mathbf{S A})_{1} \cdot \mathbf{W}_{1}$} & $\mathrm{C}=\mathrm{O} \ldots \mathrm{H}-\mathrm{O}-\mathrm{S}$ & 1.712 & 0.0410 & 0.1479 \\
\hline & $\mathrm{C}-\mathrm{O}-\mathrm{H} \ldots \mathrm{O}=\mathrm{S}$ & 1.601 & 0.0531 & 0.1773 \\
\hline & $\mathrm{H}-\mathrm{O} \ldots \mathrm{H}-\mathrm{O}-\mathrm{H}$ & 1.907 & 0.0253 & 0.0959 \\
\hline \multirow[t]{2}{*}{$(\mathbf{G A})_{1} \cdot(\mathbf{S A})_{1} \cdot \mathbf{W}_{2}$} & $\mathrm{C}=\mathrm{O} \ldots \mathrm{H}-\mathrm{O}-\mathrm{S}$ & 1.603 & 0.0537 & 0.1728 \\
\hline & $\mathrm{C}-\mathrm{O}-\mathrm{H} \ldots \mathrm{O}=\mathrm{S}$ & 1.640 & 0.0472 & 0.1685 \\
\hline \multirow[t]{2}{*}{$(\mathbf{G W})_{1} \cdot(\mathbf{S A})_{1} \cdot \mathbf{W}_{2}$} & $\mathrm{C}=\mathrm{O} \ldots \mathrm{H}-\mathrm{O}-\mathrm{S}$ & 1.570 & 0.0584 & 0.1771 \\
\hline & $\mathrm{C}-\mathrm{O}-\mathrm{H} \ldots \mathrm{O}=\mathrm{S}$ & 1.656 & 0.0455 & 0.1640 \\
\hline \multirow[t]{2}{*}{$(\mathbf{G A})_{1} \cdot(\mathbf{S A})_{1} \cdot \mathbf{W}_{3}$} & $\mathrm{C}=\mathrm{O} \ldots \mathrm{H}-\mathrm{O}-\mathrm{S}$ & 1.736 & 0.0366 & 0.1446 \\
\hline & $\mathrm{C}-\mathrm{O}-\mathrm{H} \ldots \mathrm{O}=\mathrm{S}$ & 1.664 & 0.0447 & 0.1620 \\
\hline \multirow[t]{3}{*}{$(\mathbf{G W})_{1} \cdot(\mathbf{S A})_{1} \cdot \mathbf{W}_{3}$} & $\mathrm{C}=\mathrm{O} \ldots \mathrm{H}-\mathrm{O}-\mathrm{H}$ & 1.879 & 0.0267 & 0.1075 \\
\hline & $\mathrm{C}-\mathrm{O}(\mathrm{H}) \ldots \mathrm{H}-\mathrm{O}-\mathrm{H}$ & 2.061 & 0.0181 & 0.0630 \\
\hline & $\mathrm{O}-\mathrm{H} \ldots \mathrm{O}=\mathrm{S}$ & 1.728 & 0.0400 & 0.1484 \\
\hline \multirow[t]{2}{*}{$(\mathbf{G A})_{1} \cdot(\mathbf{S A})_{1} \cdot \mathbf{N}_{1}$} & $\mathrm{C}=\mathrm{O} \ldots \mathrm{H}-\mathrm{O}-\mathrm{S}$ & 1.622 & 0.0511 & 0.1694 \\
\hline & $\mathrm{C}-\mathrm{O}-\mathrm{H} \ldots \mathrm{O}=\mathrm{S}$ & 1.620 & 0.0495 & 0.1740 \\
\hline$(\mathbf{G W})_{1} \cdot(\mathbf{S A})_{1} \cdot \mathbf{N}_{1}$ & $\mathrm{C}=\mathrm{O} \ldots \mathrm{H}-\mathrm{O}-\mathrm{S}$ & 1.737 & 0.0387 & 0.1402 \\
\hline
\end{tabular}




\begin{tabular}{|c|c|c|c|c|}
\hline & $\mathrm{C}-\mathrm{O}-\mathrm{H} \ldots \mathrm{O}=\mathrm{S}$ & 1.554 & 0.0609 & 0.1815 \\
\hline & $\mathrm{H}-\mathrm{O} \ldots \mathrm{H}-\mathrm{N}-\mathrm{H}$ & 2.128 & 0.0165 & 0.0545 \\
\hline \multirow[t]{2}{*}{$(\mathbf{G A})_{1} \cdot(\mathbf{S A})_{1} \cdot \mathbf{N}_{1} \cdot \mathbf{W}_{1}$} & $\mathrm{C}=\mathrm{O} \ldots \mathrm{H}-\mathrm{O}-\mathrm{S}$ & 1.675 & 0.0451 & 0.1576 \\
\hline & $\mathrm{C}-\mathrm{O}-\mathrm{H} \ldots \mathrm{O}=\mathrm{S}$ & 1.571 & 0.0578 & 0.1790 \\
\hline \multirow[t]{3}{*}{$(\mathbf{G W})_{1} \cdot(\mathbf{S A})_{1} \cdot \mathbf{N}_{1} \cdot \mathbf{W}_{1}$} & $\mathrm{C}=\mathrm{O} \ldots \mathrm{H}-\mathrm{O}-\mathrm{S}$ & 1.869 & 0.0285 & 0.1020 \\
\hline & $\mathrm{C}-\mathrm{O}-\mathrm{H} \ldots \mathrm{O}=\mathrm{S}$ & 1.479 & 0.0775 & 0.1639 \\
\hline & $\mathrm{H}-\mathrm{O} \ldots \mathrm{H}-\mathrm{N}-\mathrm{H}$ & 1.994 & 0.0219 & 0.0760 \\
\hline \multirow[t]{2}{*}{$(\mathbf{G A})_{1} \cdot(\mathbf{S A})_{1} \cdot \mathbf{N}_{1} \cdot \mathbf{W}_{2}$} & $\mathrm{C}=\mathrm{O} \ldots \mathrm{H}-\mathrm{O}-\mathrm{H}$ & 1.889 & 0.0259 & 0.0988 \\
\hline & $\mathrm{C}-\mathrm{O}-\mathrm{H} \ldots \mathrm{O}=\mathrm{S}$ & 1.407 & 0.0927 & 0.1261 \\
\hline \multirow[t]{3}{*}{$(\mathbf{G W})_{1} \cdot(\mathbf{S A})_{1} \cdot \mathbf{N}_{1} \cdot \mathbf{W}_{2}$} & $\mathrm{C}=\mathrm{O} \ldots \mathrm{H}-\mathrm{O}-\mathrm{S}$ & 1.929 & 0.0250 & 0.0868 \\
\hline & $\mathrm{C}-\mathrm{O}-\mathrm{H} \ldots \mathrm{O}=\mathrm{S}$ & 1.486 & 0.0758 & 0.1695 \\
\hline & $\mathrm{H}-\mathrm{O} \ldots \mathrm{H}-\mathrm{N}-\mathrm{H}$ & 2.063 & 0.0192 & 0.0648 \\
\hline \multirow[t]{2}{*}{$(\mathbf{G A})_{1} \cdot(\mathbf{S A})_{1} \cdot \mathbf{N}_{1} \cdot \mathbf{W}_{3}$} & $\mathrm{C}=\mathrm{O} \ldots \mathrm{H}-\mathrm{O}-\mathrm{H}$ & 1.843 & 0.0287 & 0.1119 \\
\hline & $\mathrm{C}-\mathrm{O}-\mathrm{H} \ldots \mathrm{O}=\mathrm{S}$ & 1.461 & 0.0803 & 0.1589 \\
\hline \multirow{3}{*}{$(\mathbf{G W})_{1} \cdot(\mathbf{S A})_{1} \cdot \mathbf{N}_{1} \cdot \mathbf{W}_{3}$} & $\mathrm{C}=\mathrm{O} \ldots \mathrm{H}-\mathrm{O}-\mathrm{H}$ & 1.831 & 0.0295 & 0.1152 \\
\hline & $\mathrm{C}-\mathrm{O}-\mathrm{H} \ldots \mathrm{O}=\mathrm{S}$ & 1.475 & 0.0769 & 0.1658 \\
\hline & $\mathrm{H}-\mathrm{O} \ldots \mathrm{H}-\mathrm{N}-\mathrm{H}$ & 1.991 & 0.0222 & 0.0771 \\
\hline \multirow[t]{2}{*}{$(\mathbf{G A})_{1} \cdot(\mathbf{S A})_{1} \cdot \mathbf{N}_{1} \cdot \mathbf{W}_{4}$} & $\mathrm{C}=\mathrm{O} \ldots \mathrm{H}-\mathrm{O}-\mathrm{H}$ & 2.125 & 0.0177 & 0.0583 \\
\hline & $\mathrm{C}-\mathrm{O}-\mathrm{H} \ldots \mathrm{O}=\mathrm{S}$ & 1.542 & 0.0644 & 0.1806 \\
\hline \multirow[t]{4}{*}{$(\mathbf{G W})_{1} \cdot(\mathbf{S A})_{1} \cdot \mathbf{N}_{1} \cdot \mathbf{W}_{4}$} & $\mathrm{C}=\mathrm{O} \ldots \mathrm{H}-\mathrm{O}-\mathrm{H}$ & 1.857 & 0.0183 & 0.0412 \\
\hline & $\mathrm{C}-\mathrm{O}-\mathrm{H} \ldots \mathrm{O}=\mathrm{S}$ & 1.588 & 0.0410 & 0.0584 \\
\hline & $\mathrm{H}-\mathrm{O} \ldots \mathrm{H}-\mathrm{N}-\mathrm{H}$ & 1.966 & 0.0201 & 0.0312 \\
\hline & O-H...O-H2 & 1.849 & 0.0293 & 0.0288 \\
\hline \multirow[t]{2}{*}{$(\mathbf{G A})_{1} \cdot(\mathbf{S A})_{2}$} & $\mathrm{C}=\mathrm{O} \ldots \mathrm{H}-\mathrm{O}-\mathrm{S}$ & 1.508 & 0.0690 & 0.1724 \\
\hline & $\mathrm{C}-\mathrm{O}-\mathrm{H} \ldots \mathrm{O}=\mathrm{S}$ & 1.698 & 0.0403 & 0.1521 \\
\hline \multirow[t]{3}{*}{$(\mathbf{G W})_{1} \cdot(\mathbf{S A})_{2}$} & $\mathrm{C}=\mathrm{O} \ldots \mathrm{H}-\mathrm{O}-\mathrm{S}$ & 1.912 & 0.0245 & 0.0884 \\
\hline & $\mathrm{C}=\mathrm{O} \ldots \mathrm{H}-\mathrm{O}-\mathrm{S}$ & 1.665 & 0.0455 & 0.1611 \\
\hline & $\mathrm{H}-\mathrm{O} \ldots \mathrm{H}-\mathrm{O}-\mathrm{S}$ & 1.747 & 0.0381 & 0.1384 \\
\hline$(\mathbf{G A})_{1} \cdot(\mathbf{S A})_{2} \cdot \mathbf{W}_{1}$ & $\mathrm{C}=\mathrm{O} \ldots \mathrm{H}-\mathrm{O}-\mathrm{S}$ & 1.532 & 0.0646 & 0.1760 \\
\hline
\end{tabular}




\begin{tabular}{|c|c|c|c|c|}
\hline & $\mathrm{C}-\mathrm{O}-\mathrm{H} \ldots \mathrm{O}=\mathrm{S}$ & 1.698 & 0.0402 & 0.1522 \\
\hline \multirow[t]{4}{*}{$(\mathbf{G W})_{1} \cdot(\mathbf{S A})_{2} \cdot \mathbf{W}_{1}$} & $\mathrm{C}=\mathrm{O} \ldots \mathrm{H}-\mathrm{O}-\mathrm{H}$ & 1.809 & 0.0284 & 0.1242 \\
\hline & $\mathrm{O}-\mathrm{H} \ldots \mathrm{O}=\mathrm{S}$ & 1.943 & 0.0236 & 0.0863 \\
\hline & $\mathrm{H}-\mathrm{O} \ldots \mathrm{H}-\mathrm{O}-\mathrm{S}$ & 1.551 & 0.0642 & 0.1651 \\
\hline & $\mathrm{O}-\mathrm{H} \ldots \mathrm{O}=\mathrm{S}$ & 1.751 & 0.0351 & 0.1427 \\
\hline \multirow[t]{2}{*}{$(\mathbf{G A})_{1} \cdot(\mathbf{S A})_{2} \cdot \mathbf{W}_{2}$} & $\mathrm{C}=\mathrm{O} \ldots \mathrm{H}-\mathrm{O}-\mathrm{S}$ & 1.549 & 0.0618 & 0.1768 \\
\hline & $\mathrm{C}-\mathrm{O}-\mathrm{H} \ldots \mathrm{O}=\mathrm{S}$ & 1.677 & 0.0429 & 0.1578 \\
\hline \multirow[t]{4}{*}{$(\mathbf{G W})_{1} \cdot(\mathbf{S A})_{2} \cdot \mathbf{W}_{2}$} & $\mathrm{C}=\mathrm{O} \ldots \mathrm{H}-\mathrm{O}-\mathrm{S}$ & 1.662 & 0.0444 & 0.1649 \\
\hline & $\mathrm{C}=\mathrm{O} \ldots \mathrm{H}-\mathrm{O}-\mathrm{H}$ & 2.124 & 0.0182 & 0.0573 \\
\hline & $\mathrm{H}-\mathrm{O} \ldots \mathrm{H}-\mathrm{O}-\mathrm{S}$ & 1.687 & 0.0439 & 0.1539 \\
\hline & $\mathrm{O}-\mathrm{H} \ldots \mathrm{O}=\mathrm{S}$ & 1.679 & 0.0426 & 0.1677 \\
\hline \multirow[t]{2}{*}{$(\mathbf{G A})_{1} \cdot(\mathbf{S A})_{2} \cdot \mathbf{W}_{3}$} & $\mathrm{C}=\mathrm{O} \ldots \mathrm{H}-\mathrm{O}-\mathrm{S}$ & 1.599 & 0.0545 & 0.1736 \\
\hline & $\mathrm{C}-\mathrm{O}-\mathrm{H} \ldots \mathrm{O}=\mathrm{S}$ & 1.627 & 0.0492 & 0.1706 \\
\hline \multirow[t]{5}{*}{$(\mathbf{G W})_{1} \cdot(\mathbf{S A})_{2} \cdot \mathbf{W}_{3}$} & $\mathrm{C}=\mathrm{O} \ldots \mathrm{H}-\mathrm{O}-\mathrm{S}$ & 1.570 & 0.0576 & 0.1809 \\
\hline & $\mathrm{C}=\mathrm{O} \ldots \mathrm{H}-\mathrm{O}-\mathrm{H}$ & 2.160 & 0.0180 & 0.0560 \\
\hline & C-O-H...O-H2 & 1.496 & 0.0742 & 0.1572 \\
\hline & $\mathrm{H}-\mathrm{O} \ldots \mathrm{H}-\mathrm{O}-\mathrm{S}$ & 1.657 & 0.0476 & 0.1623 \\
\hline & $\mathrm{O}-\mathrm{H} \ldots \mathrm{O}=\mathrm{S}$ & 1.691 & 0.0397 & 0.1637 \\
\hline \multirow[t]{2}{*}{$(\mathbf{G A})_{1} \cdot(\mathbf{S A})_{2} \cdot \mathbf{W}_{4}$} & $\mathrm{C}=\mathrm{O} \ldots \mathrm{H}-\mathrm{O}-\mathrm{S}$ & 1.582 & 0.0571 & 0.1753 \\
\hline & $\mathrm{C}-\mathrm{O}-\mathrm{H} \ldots \mathrm{O}=\mathrm{S}$ & 1.617 & 0.0508 & 0.1725 \\
\hline \multirow[t]{6}{*}{$(\mathbf{G W})_{1} \cdot(\mathbf{S A})_{2} \cdot \mathbf{W}_{4}$} & $\mathrm{C}=\mathrm{O} \ldots \mathrm{H}-\mathrm{O}-\mathrm{S}$ & 1.571 & 0.0579 & 0.1779 \\
\hline & C-O-H...O-H2 & 1.557 & 0.0636 & 0.1642 \\
\hline & $\mathrm{C}-\mathrm{O}(\mathrm{H}) \ldots \mathrm{H}-\mathrm{O}-\mathrm{H}$ & 2.006 & 0.0222 & 0.0743 \\
\hline & $\mathrm{O}-\mathrm{H} \ldots \mathrm{O}=\mathrm{S}$ & 1.799 & 0.0340 & 0.1277 \\
\hline & $\mathrm{O}-\mathrm{H} \ldots \mathrm{O}=\mathrm{S}$ & 1.951 & 0.0247 & 0.0834 \\
\hline & H-O...H-O-S & 1.726 & 0.0397 & 0.1458 \\
\hline \multirow{2}{*}{$(\mathbf{G W})_{1} \cdot(\mathbf{S A})_{2} \cdot \mathbf{W}_{5}$} & $\mathrm{C}=\mathrm{O} \ldots \mathrm{H}-\mathrm{O}-\mathrm{S}$ & 1.590 & 0.0558 & 0.1742 \\
\hline & $\mathrm{C}-\mathrm{O}-\mathrm{H} \ldots \mathrm{O}=\mathrm{S}$ & 1.615 & 0.0510 & 0.1731 \\
\hline
\end{tabular}


Table S85. The realistic hydration reaction conversion ratio $\left(X_{\mathbf{G A}}, \%\right)$ of $\mathbf{G A}$-based clusters at varying temperatures and ralative humidities.

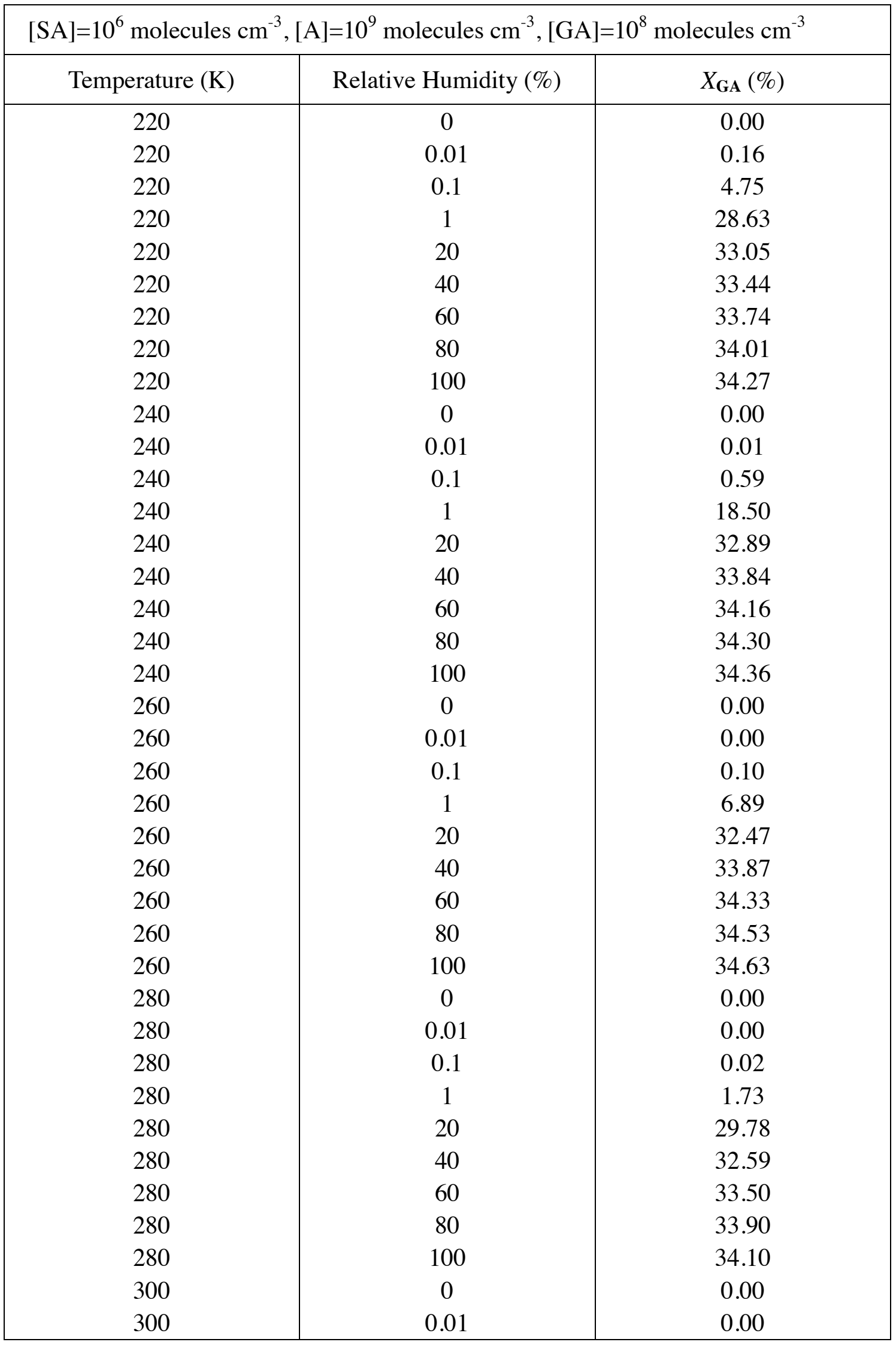




\begin{tabular}{|c|c|c|}
\hline 300 & 0.1 & 0.00 \\
300 & 1 & 0.44 \\
300 & 20 & 22.69 \\
300 & 40 & 28.13 \\
300 & 60 & 30.01 \\
300 & 80 & 30.89 \\
300 & 100 & 31.34 \\
\hline
\end{tabular}

Table S86. The realistic hydration reaction conversion ratio $\left(X_{\mathbf{G A}}, \%\right)$ of $\mathbf{G A}$-based clusters at varying ammonia and sulfuric acid concentrations.

\begin{tabular}{|c|c|c|}
\hline $0 \mathrm{~K}, \mathrm{RH}=50 \%,[\mathrm{GA}]=1$ & lecules $\mathrm{cm}^{-3}$ & \\
\hline [A] (molecules $\mathrm{cm}^{-3}$ ) & {$[\mathrm{SA}]\left(\right.$ molecules $\left.\mathrm{cm}^{-3}\right)$} & $X_{\mathbf{G A}}(\%)$ \\
\hline $10^{7}$ & $10^{4}$ & 0.52 \\
\hline $10^{7}$ & $10^{5}$ & 4.93 \\
\hline $10^{7}$ & $10^{6}$ & 34.15 \\
\hline $10^{7}$ & $10^{7}$ & 83.85 \\
\hline $10^{7}$ & $10^{8}$ & 98.13 \\
\hline $10^{8}$ & $10^{4}$ & 0.52 \\
\hline $10^{8}$ & $10^{5}$ & 4.93 \\
\hline $10^{8}$ & $10^{6}$ & 34.15 \\
\hline $10^{8}$ & $10^{7}$ & 83.85 \\
\hline $10^{8}$ & $10^{8}$ & 98.13 \\
\hline $10^{9}$ & $10^{4}$ & 0.52 \\
\hline $10^{9}$ & $10^{5}$ & 4.93 \\
\hline $10^{9}$ & $10^{6}$ & 34.15 \\
\hline $10^{9}$ & $10^{7}$ & 83.85 \\
\hline $10^{9}$ & $10^{8}$ & 98.13 \\
\hline $10^{10}$ & $10^{4}$ & 0.52 \\
\hline $10^{10}$ & $10^{5}$ & 4.93 \\
\hline $10^{10}$ & $10^{6}$ & 34.17 \\
\hline $10^{10}$ & $10^{7}$ & 83.86 \\
\hline $10^{10}$ & $10^{8}$ & 98.13 \\
\hline $10^{11}$ & $10^{4}$ & 0.52 \\
\hline $10^{11}$ & $10^{5}$ & 4.97 \\
\hline $10^{11}$ & $10^{6}$ & 34.33 \\
\hline $10^{11}$ & $10^{7}$ & 83.96 \\
\hline $10^{11}$ & $10^{8}$ & 98.13 \\
\hline
\end{tabular}


Table S87. The formation rates $\left(J, \mathrm{~cm}^{-3} \mathrm{~s}^{-1}\right)$ of $(\mathbf{G A} / \mathbf{G W})_{x} \cdot(\mathbf{S A})_{y} \cdot \mathbf{A}_{z}$ clusters with the vabriations of the concentration of GA ([GA]) at different temperatures of 220, 240, 260,280 and $300 \mathrm{~K} .[\mathbf{S A}]=10^{6}$ molecules $\mathrm{cm}^{-3} \cdot[\mathbf{A}]=10^{9}$ molecules $\mathrm{cm}^{-3} \cdot \mathrm{RH}=50 \%$.

\begin{tabular}{llllll}
\hline$[\mathbf{G A}]$ & $220 \mathrm{~K}$ & $240 \mathrm{~K}$ & $260 \mathrm{~K}$ & $280 \mathrm{~K}$ & $300 \mathrm{~K}$ \\
\hline $10^{7}$ & $1.05 \times 10^{2}$ & $1.92 \times 10^{1}$ & $2.09 \times 10^{-2}$ & $5.59 \times 10^{-5}$ & $3.69 \times 10^{-7}$ \\
$10^{8}$ & $1.13 \times 10^{2}$ & $1.92 \times 10^{1}$ & $2.09 \times 10^{-2}$ & $5.59 \times 10^{-5}$ & $3.69 \times 10^{-7}$ \\
$10^{9}$ & $2.04 \times 10^{2}$ & $1.94 \times 10^{1}$ & $2.09 \times 10^{-2}$ & $5.59 \times 10^{-5}$ & $3.69 \times 10^{-7}$ \\
$10^{10}$ & $2.89 \times 10^{3}$ & $2.10 \times 10^{1}$ & $2.10 \times 10^{-2}$ & $5.59 \times 10^{-5}$ & $3.69 \times 10^{-7}$ \\
$10^{11}$ & $2.09 \times 10^{5}$ & $4.24 \times 10^{1}$ & $2.17 \times 10^{-2}$ & $5.61 \times 10^{-5}$ & $3.69 \times 10^{-7}$ \\
\hline
\end{tabular}

Table S88. The formation rates $\left(J, \mathrm{~cm}^{-3} \mathrm{~s}^{-1}\right)$ of $(\mathbf{G A} / \mathbf{G W})_{x} \cdot(\mathbf{S A})_{y} \cdot \mathbf{A}_{z}$ clusters with the vabriations of the concentration of GA ([GA]) at different relative humidities (RH) of $20 \%, 40 \%, 60 \%, 80 \%$ and $100 \%$. [SA $]=10^{6}$ molecules $\mathrm{cm}^{-3} \cdot[\mathbf{A}]=10^{9}$ molecules $\mathrm{cm}^{-3}$. $\mathrm{T}=220 \mathrm{~K}$

\begin{tabular}{llllll}
\hline$[\mathbf{G A}]$ & $20 \%$ & $40 \%$ & $60 \%$ & $80 \%$ & $100 \%$ \\
\hline $10^{7}$ & $1.03 \times 10^{2}$ & $1.04 \times 10^{2}$ & $1.06 \times 10^{2}$ & $1.07 \times 10^{2}$ & $1.09 \times 10^{2}$ \\
$10^{8}$ & $1.10 \times 10^{2}$ & $1.12 \times 10^{2}$ & $1.14 \times 10^{2}$ & $1.17 \times 10^{2}$ & $1.21 \times 10^{2}$ \\
$10^{9}$ & $1.91 \times 10^{2}$ & $1.96 \times 10^{2}$ & $2.11 \times 10^{2}$ & $2.45 \times 10^{2}$ & $2.76 \times 10^{2}$ \\
$10^{10}$ & $2.43 \times 10^{3}$ & $2.58 \times 10^{3}$ & $3.15 \times 10^{3}$ & $4.53 \times 10^{3}$ & $5.97 \times 10^{3}$ \\
$10^{11}$ & $1.69 \times 10^{5}$ & $1.83 \times 10^{5}$ & $2.30 \times 10^{5}$ & $3.37 \times 10^{5}$ & $4.39 \times 10^{5}$ \\
\hline
\end{tabular}

Table S89. The formation rates $\left(J, \mathrm{~cm}^{-3} \mathrm{~s}^{-1}\right)$ of $(\mathbf{G A} / \mathbf{G W})_{x} \cdot(\mathbf{S A})_{y} \cdot \mathbf{A}_{z}$ clusters with the vabriations of the concentration of SA ([SA]) and A ([A]) at $220 \mathrm{~K}$. $\mathrm{RH}=50 \%$. [GA $]=10^{9}$ molecules $\mathrm{cm}^{-3}$.

\begin{tabular}{|c|c|c|c|c|c|}
\hline$[\mathrm{SA}] \quad[\mathrm{A}]$ & $10^{7}$ & $10^{8}$ & $10^{9}$ & $10^{10}$ & $10^{11}$ \\
\hline $10^{4}$ & $1.31 \times 10^{-4}$ & $2.50 \times 10^{-4}$ & $3.44 \times 10^{-4}$ & $1.80 \times 10^{-3}$ & $2.27 \times 10^{-1}$ \\
\hline $10^{5}$ & $1.29 \times 10^{-1}$ & $2.39 \times 10^{-1}$ & $3.27 \times 10^{-1}$ & 1.65 & $1.47 \times 10^{2}$ \\
\hline $10^{6}$ & $9.99 \times 10^{1}$ & $1.57 \times 10^{2}$ & $2.02 \times 10^{2}$ & $7.91 \times 10^{2}$ & $2.77 \times 10^{4}$ \\
\hline $10^{7}$ & $2.02 \times 10^{4}$ & $3.03 \times 10^{4}$ & $3.73 \times 10^{4}$ & $1.15 \times 10^{5}$ & $2.12 \times 10^{6}$ \\
\hline
\end{tabular}




\begin{tabular}{llllll}
\hline $10^{8}$ & $7.28 \times 10^{5}$ & $2.68 \times 10^{6}$ & $3.87 \times 10^{6}$ & $9.92 \times 10^{6}$ & $8.17 \times 10^{7}$ \\
\hline
\end{tabular}

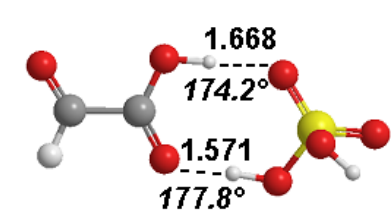

$(\mathrm{GA})_{1}(\mathrm{SA})_{1}$

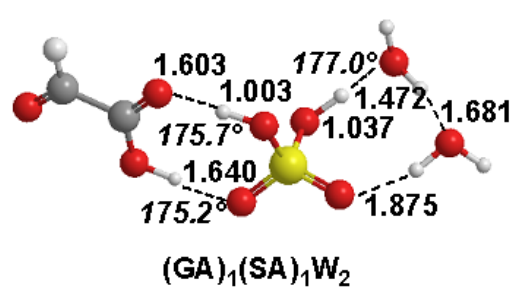

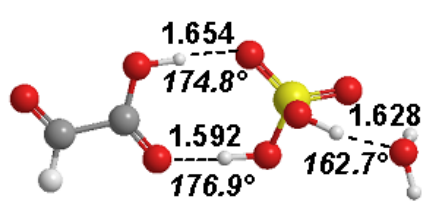

$(\mathrm{GA})_{1}(\mathrm{SA})_{1} \mathrm{~W}_{1}$

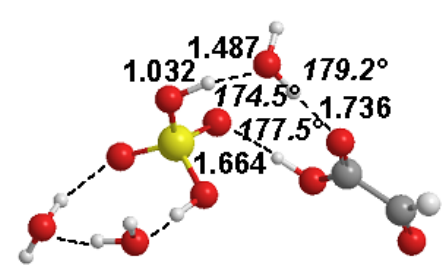

$(\mathrm{GA})_{1}(\mathrm{SA})_{1} \mathrm{~W}_{3}$

Figure S1. Most stable configuration of clusters $(\mathbf{G A})_{\mathbf{1}} \cdot(\mathbf{S A})_{\mathbf{1}} \cdot \mathbf{W}_{\mathbf{n}}(\mathrm{n}=0-3)$. The lengths of the hydrogen bonds are given in $\AA$. The hydrogen bonds are shown as dashed lines.

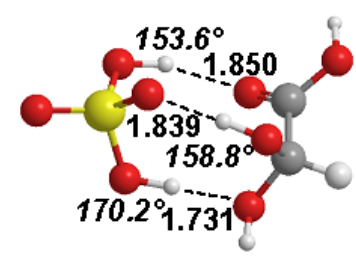

$(\mathrm{GW})_{1}(\mathrm{SA})_{1}$

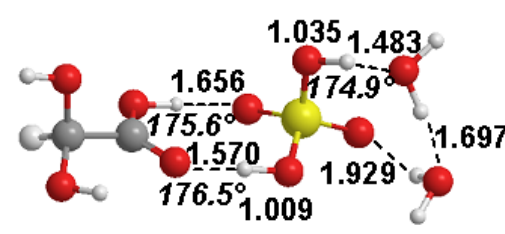

$(\mathrm{GW})_{1}(\mathrm{SA})_{1} \mathrm{~W}_{2}$

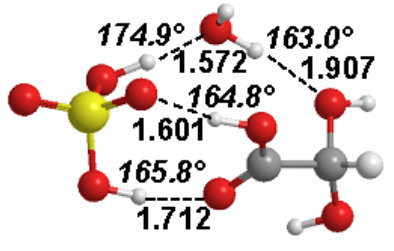

$(\mathrm{GW})_{1}(\mathrm{SA})_{1} \mathrm{~W}_{1}$

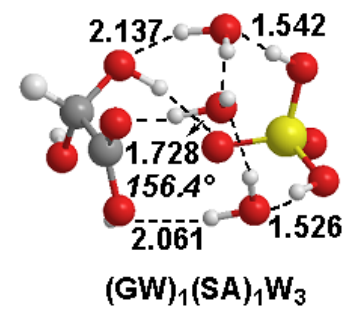

Figure S2. Most stable configuration of clusters $(\mathbf{G W})_{\mathbf{1}} \cdot(\mathbf{S A})_{\mathbf{1}} \cdot \mathbf{W}_{\mathbf{n}}(\mathrm{n}=0-3)$. The lengths of the hydrogen bonds are given in $\AA$. The hydrogen bonds are shown as dashed lines. 


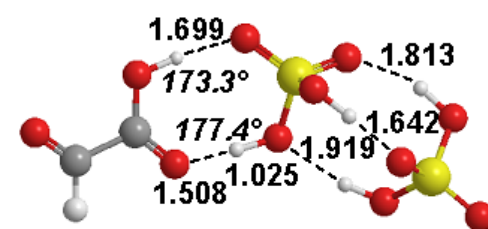

$(\mathrm{GA})_{1}(\mathrm{SA})_{2}$

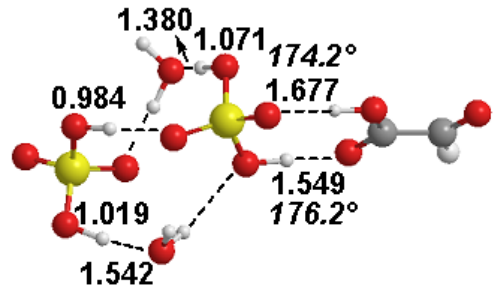

$(\mathrm{GA})_{1}(\mathrm{SA})_{2} \mathrm{~W}_{2}$

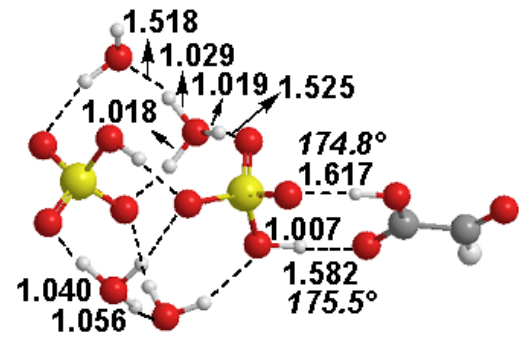

$(\mathrm{GA})_{1}(\mathrm{SA})_{2} \mathrm{~W}_{4}$

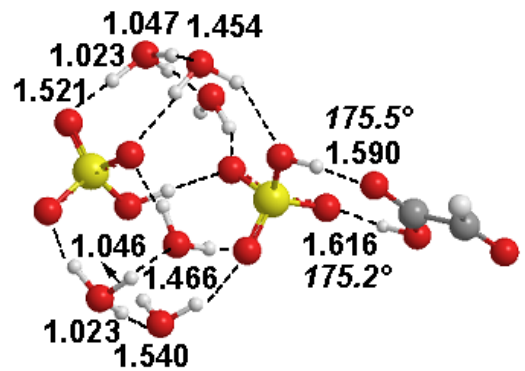

$(\mathrm{GA})_{1}(\mathrm{SA})_{2} \mathrm{~W}_{6}$

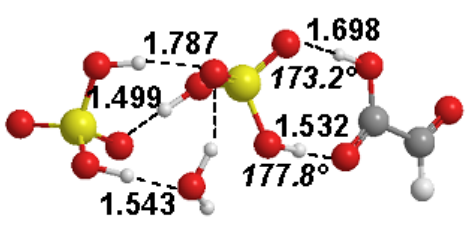

(GA) 1 (SA) ${ }_{2} \mathrm{~W}_{1}$

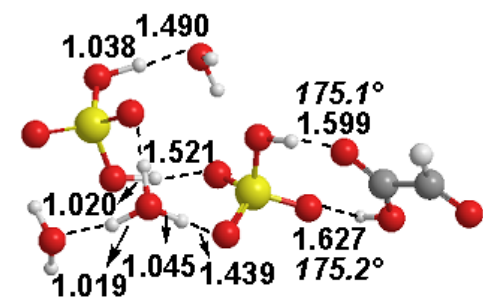

$(\mathrm{GA})_{1}(\mathrm{SA})_{2} \mathrm{~W}_{3}$

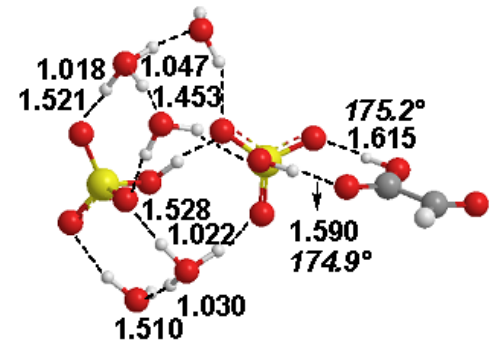

$(\mathrm{GA})_{1}(\mathrm{SA})_{2} \mathrm{~W}_{5}$

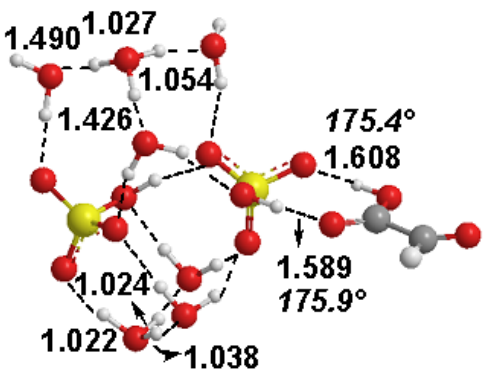

$(\mathrm{GA})_{1}(\mathrm{SA})_{2} \mathrm{~W}_{7}$

Figure S3. Most stable configuration of clusters $(\mathbf{G A})_{1} \cdot(\mathbf{S A})_{2} \cdot \mathbf{W}_{\mathbf{n}}(\mathrm{n}=0-6)$. The lengths of the hydrogen bonds are given in $\AA$. The hydrogen bonds are shown as dashed lines.

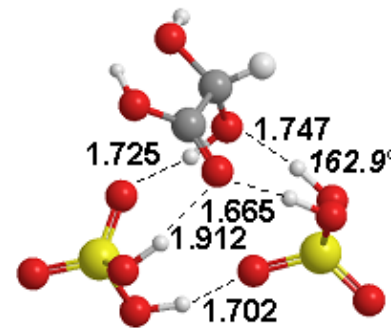

$(\mathrm{GW})_{1}(\mathrm{SA})_{2}$

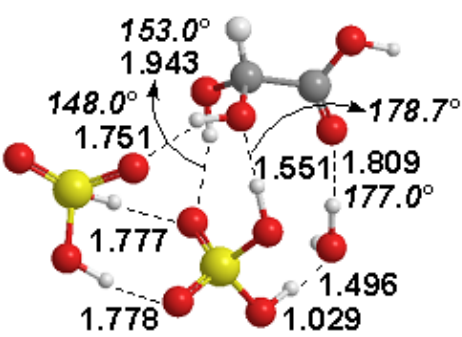

$(\mathrm{GW})_{1}(\mathrm{SA})_{2} \mathrm{~W}_{1}$

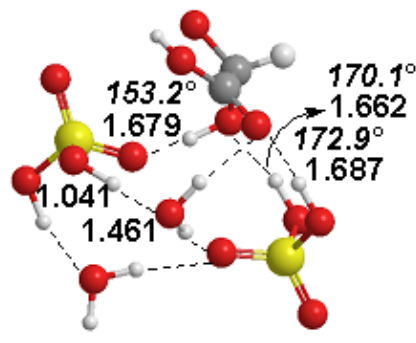

$(\mathrm{GW})_{1}(\mathrm{SA})_{2} \mathrm{~W}_{2}$ 

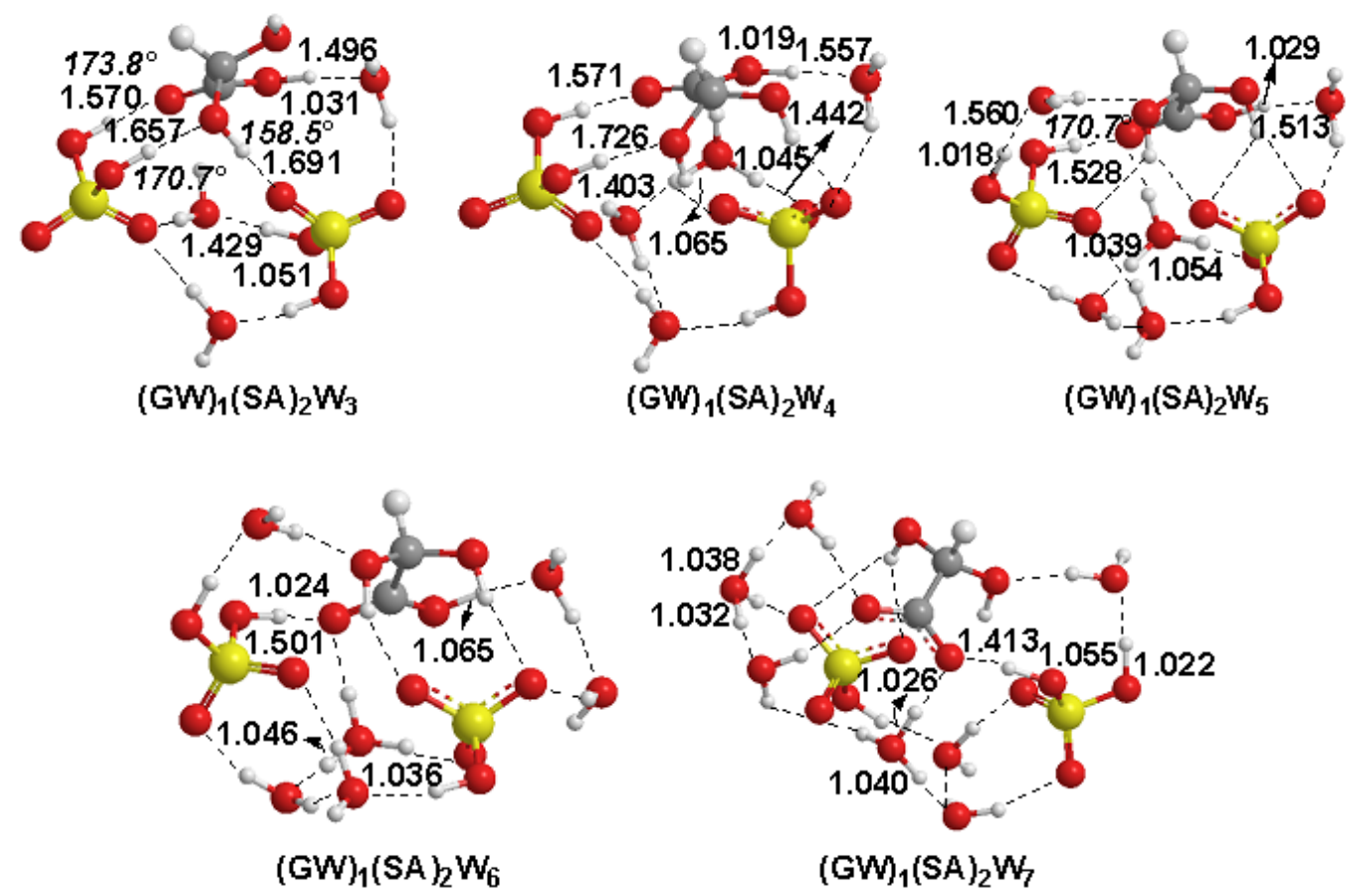

Figure S4. Most stable configuration of clusters $(\mathbf{G W})_{1} \cdot(\mathbf{S A})_{2} \cdot \mathbf{W}_{\mathbf{n}}(\mathrm{n}=0-7)$. The lengths of the hydrogen bonds are given in $\AA$. The hydrogen bonds are shown as dashed lines.
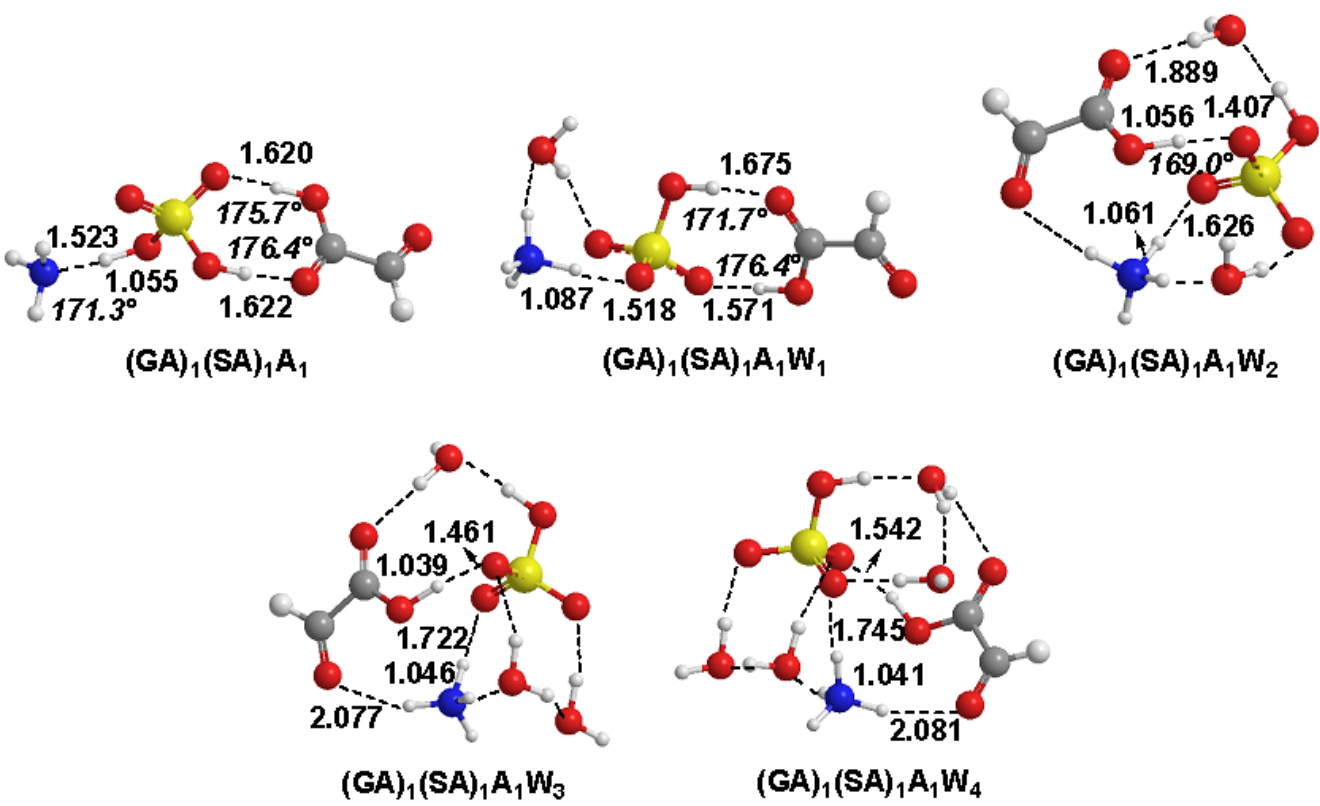

Figure S5. Most stable configuration of clusters $(\mathbf{G A})_{\mathbf{1}} \cdot(\mathbf{S A})_{\mathbf{1}} \cdot \mathbf{A}_{\mathbf{1}} \cdot \mathbf{W}_{\mathbf{n}}(\mathrm{n}=0-4)$. The lengths of the hydrogen bonds are given in $\AA$. The hydrogen bonds are shown as dashed lines. 


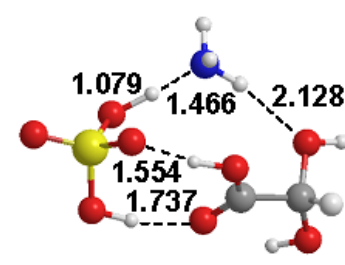

$(\mathrm{GW})_{1}(\mathrm{SA})_{1} \mathrm{~A}_{1}$

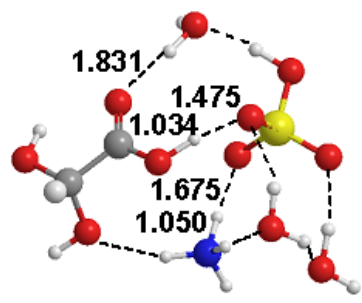

$(\mathrm{GW})_{1}(\mathrm{SA})_{1} \mathrm{~A}_{1} \mathrm{~W}_{3}$

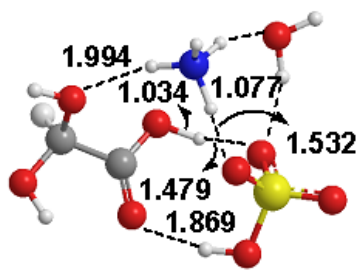

$(\mathrm{GW})_{1}(\mathrm{SA})_{1} \mathrm{~A}_{1} \mathrm{~W}_{1}$

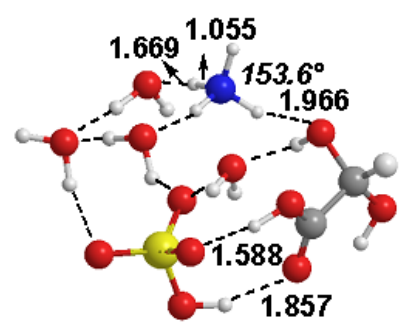

$(\mathrm{GW})_{1}(\mathrm{SA})_{1} \mathrm{~A}_{1} \mathrm{~W}_{4}$

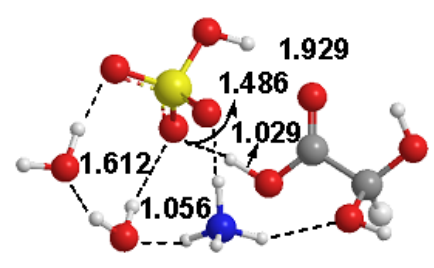

$(\mathrm{GW})_{1}(\mathrm{SA})_{1} \mathrm{~A}_{1} \mathrm{~W}_{2}$

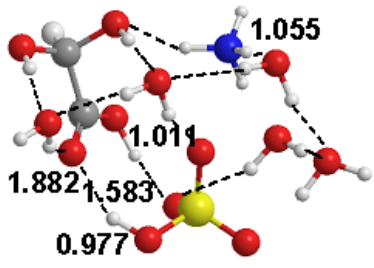

$(\mathrm{GW})_{1}(\mathrm{SA})_{1} \mathrm{~A}_{1} \mathrm{~W}_{5}$

Figure S6. Most stable configuration of clusters $(\mathbf{G W})_{\mathbf{1}} \cdot(\mathbf{S A})_{\mathbf{1}} \cdot \mathbf{A}_{\mathbf{1}} \cdot \mathbf{W}_{\mathbf{n}}(\mathrm{n}=0-5)$. The lengths of the hydrogen bonds are given in $\AA$. The hydrogen bonds are shown as dashed lines.

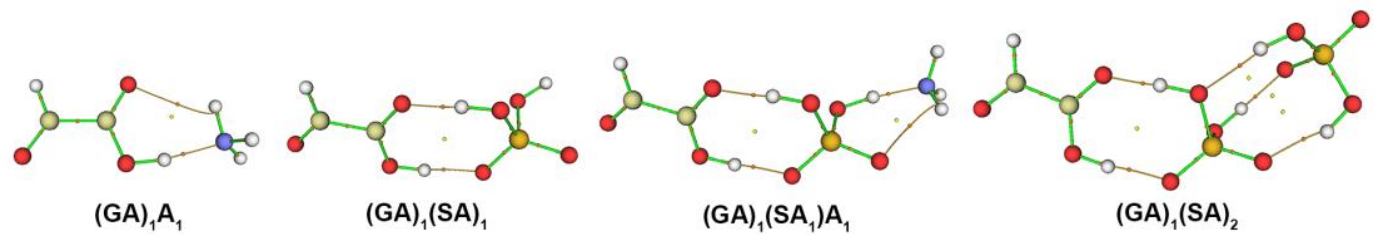

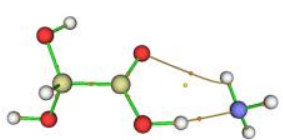

$(\mathrm{GW})_{1} \mathrm{~A}_{1}$

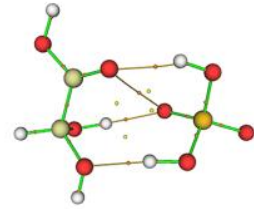

$(\mathrm{GW})_{1}(\mathrm{SA})_{1}$

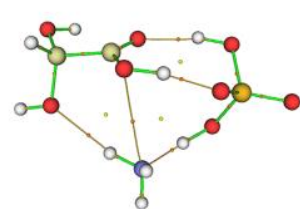

$(\mathrm{GW})_{1}(\mathrm{SA})_{1} \mathrm{~A}_{1}$

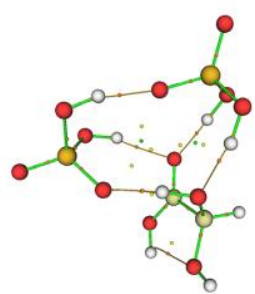

$(\mathrm{GW})_{1}(\mathrm{SA})_{2}$

Figure S7. The AIM plots of the unhydrated clusters calculated at the M06-2X/6-311++g(3df,3pd) level. The bond critical points and ring critical points are presented by the ginger and yellow balls, respectively. 

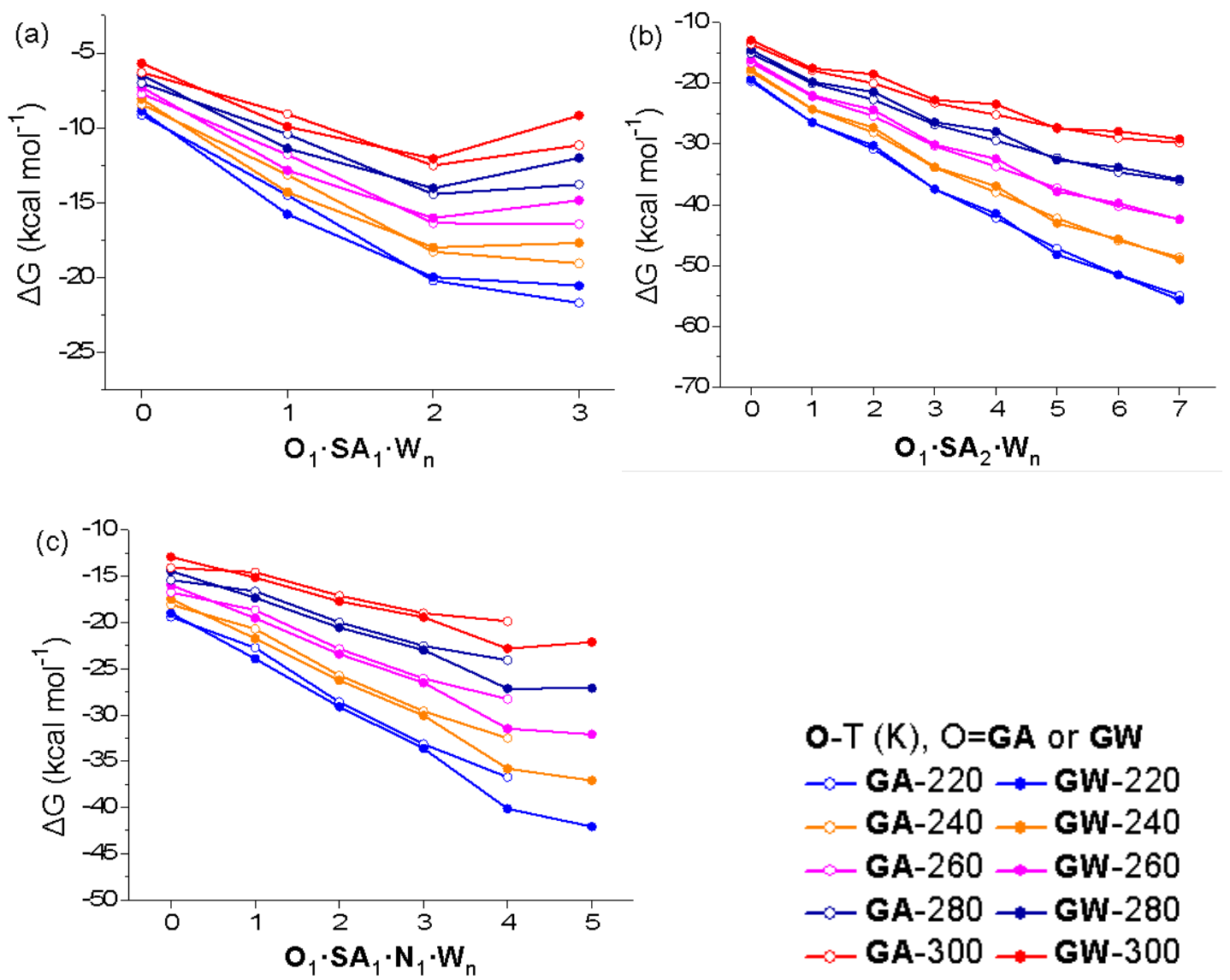

Figure S8. Gibbs free energies ( $\mathrm{kcal} \mathrm{mol}^{-1}$ ) of formation of clusters (a) $\mathbf{O}_{1} \cdot \mathbf{S} \mathbf{A}_{1} \cdot \mathbf{W}_{\mathrm{n}}$, (b) $\mathbf{O}_{1} \cdot \mathbf{S A}_{2} \cdot \mathbf{W}_{\mathrm{n}}$ and (c) $\mathbf{O}_{1} \cdot \mathbf{S A} \cdot \mathbf{N}_{1} \cdot \mathbf{W}_{\mathrm{n}} . \mathbf{O}$ indicates $\mathbf{G A}$ or $\mathbf{G W}$.

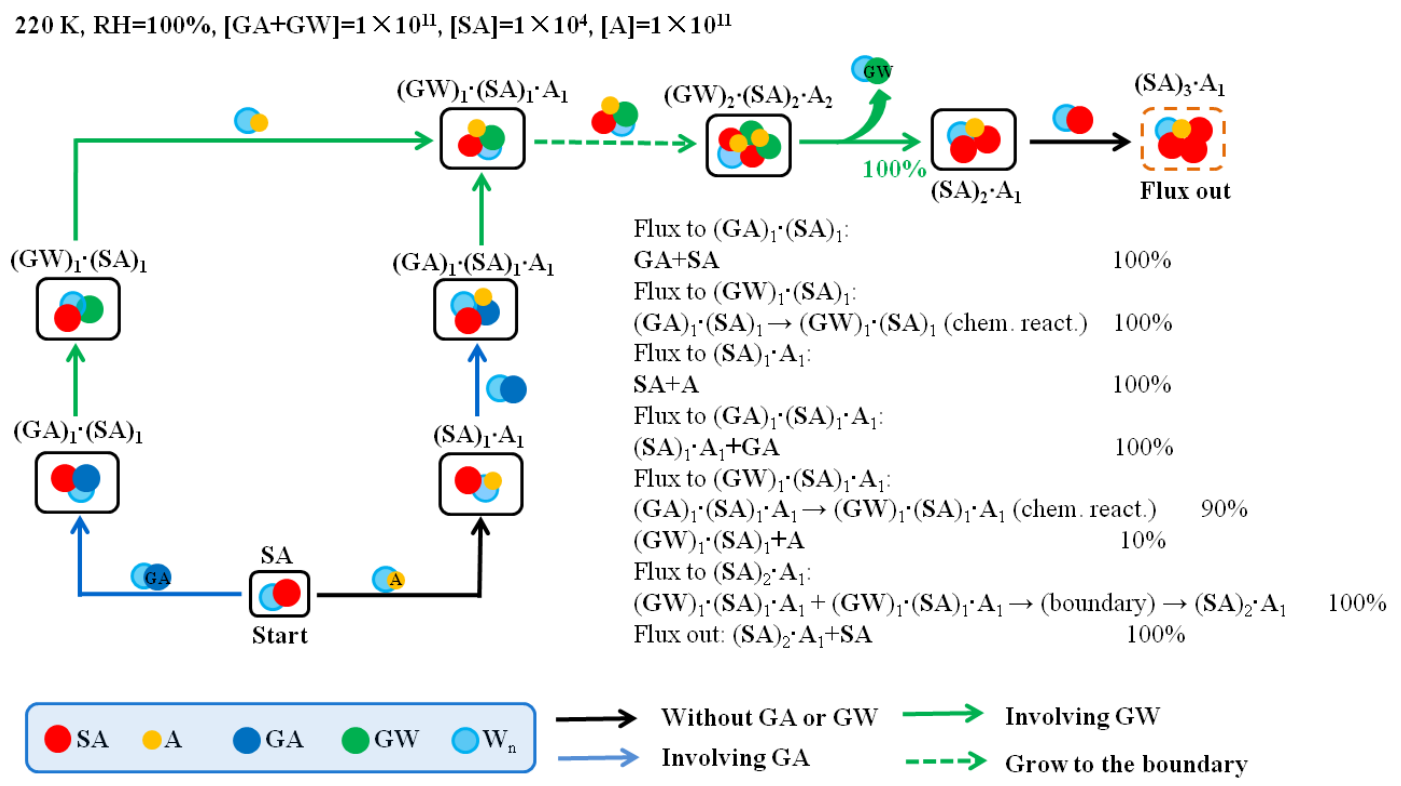

Figure S9. Main cluster formation pathways considering the hydration reaction of GA 
forming $\mathbf{G W}$ are represented by arrows. Relative amounts of clusters formed via dominating growth pathways are indicated in the side table.

\section{Section 2. Boundary Conditions}

The boundary conditions require the outgrowing clusters to have a favorable composition so that the clusters leaving the studied size range are stable enough not to evaporate back immediately. The sum of all evaporation rate coefficients (Table S5) corresponding to different evaporating molecules from the clusters involving GA or GW are relatively high. The ammonia molecule also easily evaporates from cluster $(\mathbf{S A})_{2} \cdot \mathbf{A}_{2}{ }^{2}{ }^{2}$ Thus, clusters involving molecule $\mathbf{G A}$ or $\mathbf{G W}$ and cluster $(\mathbf{S A})_{2} \cdot \mathbf{A}_{2}$ can not be considered stable enough to grow out of the studied cluster sizes. In contrast, the cluster $(\mathbf{S A})_{3} \cdot \mathbf{A}_{1}$, with a maximum evaporation rate coefficient of $55 \mathrm{~s}^{-1}$ at $300 \mathrm{~K}$, is relatively stable enough to resist evaporation. Thus, the boundary condition was set to be the cluster $(\mathbf{S A})_{3} \cdot \mathbf{A}_{1}$. Using this relatively small cluster as a boundary condition might overestimate absolute NPF rates, but it is probably sufficient for probing the relative effect of $\mathbf{G A} / \mathbf{G W}$ on cluster formation rate, which is the purpose of this study.

\section{Complete Gaussian 09 reference (Reference 52)}

Gaussian 09, Revision A.1,

Frisch, M.J., Trucks, G.W., Schlegel, H.B., Scuseria, G.E., Robb, M.A., Cheeseman, J.R., Scalmani, G., Barone, V., Mennucci, B., Petersson, G.A., Nakatsuji, H., Caricato, M., Li, X., Hratchian, H.P., Izmaylov, A.F., Bloino, J., Zheng, G., Sonnenberg, J.L., Hada, M., Ehara, M., Toyota, K., Fukuda, R., Hasegawa, J., Ishida, M., Nakajima, T., Honda, Y., Kitao, O., Nakai, H., Vreven, T., Montgomery, J.A., Peralta, J.E., Ogliaro, F., Bearpark, M., Heyd, J.J., Brothers, E., 
Kudin, K.N., Staroverov, V.N., Kobayashi, R., Normand, J., Raghavachari, K., Rendell, A., Burant, J.C., Iyengar, S.S., Tomasi, J., Cossi, M., Rega, N., Millam, J.M., Klene, M., Knox, J.E., Cross, J.B., Bakken, V., Adamo, C., Jaramillo, J., Gomperts, R., Stratmann, R.E., Yazyev, O., Austin, A.J., Cammi, R., Pomelli, C., Ochterski, J.W., Martin, R.L., Morokuma, K., Zakrzewski, V.G., Voth, G.A., Salvador, P., Dannenberg, J.J., Dapprich, S., Daniels, A.D., Farkas, O., Foresman, J.B., Ortiz, J.V., Cioslowski, J., Fox, D.J., 2009. Gaussian 09, Revision A.1, Gaussian Inc. Wallingford CT.

\section{Reference}

1 Ling Liu, Xiuhui Zhang, Zesheng Li, Yunhong Zhang and Maofa Ge. Gas-phase Hydration of Glyoxylic Acid: Kinetics and Atmospheric implications. Chemosphere. 2017, 186, 430-437.

2 I. K. Ortega, O. Kupiainen, T. Kurtén, T. Olenius, O. Wilkman, M. J. McGrath, V. Loukonen, and H. Vehkamäki. Atmos Chem Phys, 2012, 12, 225-235. 